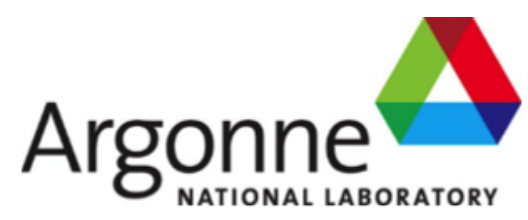

\title{
Initial study of notch sensitivity of Grade 91 using mechanisms motivated crystal plasticity finite element method
}

Applied Materials Division 
About Argonne National Laboratory

Argonne is a U.S. Department of Energy laboratory managed by UChicago Argonne, LLC under contract DE-AC02-06CH11357. The Laboratory's main facility is outside Chicago, at 9700 South Cass Avenue, Argonne, Illinois 60439. For information about Argonne and its pioneering science and technology programs, see www.anl.gov.

\section{DOCUMENT AVAILABILITY}

Online Access: U.S. Department of Energy (DOE) reports produced after 1991 and a growing number of pre-1991 documents are available free at OSTI.GOV (http://www.osti.gov/), a service of the U.S. Dept. of Energy's Office of Scientific and Technical Information

Reports not in digital format may be purchased by the public from the

National Technical Information Service (NTIS):

U.S. Department of Commerce

National Technical Information Service

5301 Shawnee Rd

Alexandria, VA 22312

www.ntis.gov

Phone: (800) 553-NTIS (6847) or (703) 605-6000

Fax: (703) 605-6900

Email: orders@ntis.gov

Reports not in digital format are available to DOE and DOE contractors from the Office of Scientific and Technical Information (OSTI)

U.S. Department of Energy

Office of Scientific and Technical Information

P.O. Box 62

Oak Ridge, TN 37831-0062

www.osti.gov

Phone: (865) 576-8401

Fax: (865) 576-5728

Email: reports@osti.gov

\section{Disclaimer}

This report was prepared as an account of work sponsored by an agency of the United States Government. Neither the United States Government nor any agency thereof, nor UChicago Argonne, LLC, nor any of their employees or officers, makes any warranty, express or implied, or assumes any legal liability or responsibility for the accuracy, completeness, or usefulness of any information, apparatus, product, or process disclosed, or represents that its use would not infringe privately owned rights. Reference herein to any specific commercial product, process, or service by trade name, trademark, manufacturer, or otherwise, does not necessarily constitute or imply its endorsement, recommendation, or favoring by the United States Government or any agency thereof. The views and opinions of document authors expressed herein do not necessarily state or reflect those of the United States Government or any agency thereof, Argonne National Laboratory, or UChicago Argonne, LLC. 


\section{Initial study of notch sensitivity of Grade 91 using mechanisms motivated crystal plasticity finite element method}

Applied Materials Division

Argonne National Laboratory

September 2019

Prepared by

A. Rovinelli, Argonne National Laboratory M. C. Messner, Argonne National Laboratory Guosheng Ye, Argonne National Laboratory T.-L. Sham, Argonne National Laboratory 

Initial study of notch sensitivity of Grade 91 using mechanisms motivated crystal plasticity finite element method

September 2019

\section{Abstract}

This report describes the development of a crystal plasticity finite element method (CPFEM) model for the multiaxial creep rupture of Grade 91 steel. The report demonstrates that core blocks in heat pipe reactor designs will see significantly different stress states than those typically found in conventional advanced reactor components where structures can often be represented as thin-walled pressure vessels. Current design practice uses effective stress measures calibrated to biaxial creep test data to account for the effect of stress multiaxiality on rupture life. Because these models rely on biaxial data they may not be accurate for the truly 3D, triaxial states found in heat pipe microreactor core blocks. A physically-based model, like the CPFEM model developed here, may be able to more accurately represent creep rupture under triaxial stresses when compared to conventional, empirical methods. This report describes the development, implementation, and validation of such a model and then applies the model to predict the effect of stress triaxiality on rupture in Grade 91 steel. The model results are used to assess the effective stress measures currently used in high temperature design codes and makes specific recommendations on improving these effective stresses to better account for the effect of 3D stress states on rupture in Grade 91. The validation and then implementation of these recommendations in design codes and standards could lead to safer, more effective heat pipe microreactor core block designs. 

Initial study of notch sensitivity of Grade 91 using mechanisms motivated crystal plasticity finite element method

September 2019

\section{Table of Contents}

Abstract

Table of Contents $\quad$ iii

List of Figures $\quad$ V

List of Tables $\quad$ ix

1 Introduction 1

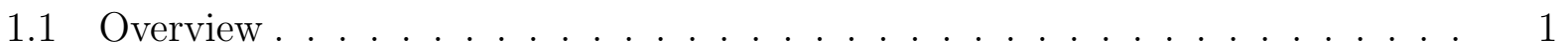

1.2 Brief survey of multiaxial creep . . . . . . . . . . . . . . . . . . . . . . . . . 4

1.3 Organization of this report . . . . . . . . . . . . . . . 5

2 Assessing the multiaxial stress state in a core block design $\quad 7$

3 Improvements to the grain bulk model 11

3.1 Brief overview of CPFEM . . . . . . . . . . . . . . . . . . . 11

3.2 Model implemented in NEML/MOOSE . . . . . . . . . . . . . . . . . . 13

3.2 .1 Kinematics . . . . . . . . . . . . . . . . . 13

3.2 .2 Constitutive models . . . . . . . . . . . . . . . . . . 16

3.2 .3 Validation . . . . . . . . . . . . . . . . . . 17

3.3 Modifications to MOOSE for large deformations . . . . . . . . . . . . . 18

4 Implementation and improvements of the grain boundary cavitation model 19

4.1 Small-strain cohesive zone framework in MOOSE . . . . . . . . . . . . . . 19

4.2 Implementation and improvement of the grain boundary cavitation model . . 20

4.2.1 Model improvements .................... . . . 24

4.2.2 Numerical implementation . . . . . . . . . . . . . . . . . . . . . 29

4.2 .3 Interface model parameters . . . . . . . . . . . . . . . . . . . 32

4.3 Model validation and testing . . . . . . . . . . . . . . . . . . 33

$5 \quad$ Effective stress measure for triaxial loading $\quad 47$

5.1 Simulations setup and rupture time . . . . . . . . . . . . . . . . . 48

5.2 Effective stresses calibration . . . . . . . . . . . . . . . . . . 50

5.3 Effective stresses parameters calibration . . . . . . . . . . . . 51

5.4 Results . . . . . . . . . . . . . . . . . . . 52

6 Conclusions 63

Acknowledgments $\quad 65$

$\begin{array}{ll}\text { Bibliography } & 67\end{array}$

A Stress analysis of a core-block concept $\quad 73$ 

Initial study of notch sensitivity of Grade 91 using mechanisms motivated crystal plasticity finite element method

September 2019

\section{List of Figures}

1.1 Standard uniaxial creep specimen. The smooth transition to the gauge region in the center of the specimen ensures the stress state in the critical region is uniaxial. Photo used with permission from Y. Wang, Oak Ridge National Laboratory. . . . . . . . . . . . . . . . . . . . . .

1.2 Typical pressurized creep specimen tested to a failure. The thin-walled capped tube is pressurized which induces a biaxial stress state in tubular shell. The principal stresses are, approximately, $\sigma_{1}=2 \sigma_{2}$ with $\sigma_{3}=0$. Figure reproduced from $[52] \ldots \ldots \ldots \ldots \ldots$

2.1 Finite element mesh used in the calculation. . . . . . . . . . . . . . . 7

2.2 Representative temperature field near the center of the modeled region. . . . 8

2.3 Representative triaxiality factors (Eq. 2.1) near the center of the modeled region. . . . . . . . . . . . . . . . . . . .

3.1 Example CPFEM analysis of a cube of FCC material undergoing $50 \%$ rolling strain. (a) Initial grain orientations and the problem mesh. (b) Final grain orientations. (c) von Mises stress field from the final simulation step. . . . .

3.2 Description of the frames of reference in the standard crystal plasticity kinematics. . . . . . . . . . . . . . . . . . .

3.3 Validation test applying 100\% rolling deformation to an FCC polycrystal with an initially random texture. For each column of figures the top figure shows the initial texture and the bottom figure shows the texture after deformation.

4.1 Schematic representing the geometrical quantities used in the grain boundary cavitation model and their physical meaning. $a$ : void half radius, $b$ : void-tovoid half distance, and $\Psi$ : cavity half tip angle. . . . . . . . . . . . . .

4.2 a): schematic representing the location of cavities on the grain boundary plane assuming axial symmetry; b) Schematic depicting the axisymmetric model used to identify the interface thickness, including the imposed boundary conditions. . . . . . . . . . . . . . . . . . .

4.3 Contour plot depicting the von Mises stress gradient field for different $a / b$ ratios. For a cavity with $a / b=0.98$ the stress becomes constant after a distance $3 b$ from the grain boundary plane. . . . . . . . . . . . . .

4.4 The two element mesh utilized to check the model behavior. On the left the mesh is colored by grain number, on the right the interface between the two grains is highlighted in green.

4.5 Results of the 2 element uniaxial cyclic test. a) cavity half radius $a$ and normal traction $T_{N}$ versus time, b) cavity half spacing $b$ and normal traction $T_{N}$ versus time, c) interface strain and grain elastic strain versus time. . . .

4.6 Results for the 2 element, stress-controlled simulation utilizing only the $\dot{V}_{L}$ term: a) final configuration after rupture (displacements are reduced by a factor of 1000), b) traction and the interface displacement jump versus time and c) the interface damage and the interface displacement jump versus time. 
Initial study of notch sensitivity of Grade 91 using mechanisms motivated crystal plasticity finite element method

September 2019

4.7 Results for the 2 element, stress-controlled simulation utilizing only the $\dot{V}_{H}$ term: a) final configuration after rupture (displacements are reduced by a factor of 1000), b) traction and the interface displacement jump versus time and c) the interface damage and the interface displacement jump versus time.

4.8 Results for the 2 element, stress-controlled simulation utilizing both $\dot{V}_{L}$ and $\dot{V}_{H}$ terms: a) final configuration after rupture (displacements are reduced by a factor of 1000), b) traction and the interface displacement jump versus time and c) the interface damage and the interface displacement jump versus time.

4.9 Detailed results for the interface quadrature point exhibiting a damage increase under compression: a) damage accumulation, b) normal traction, c) interface separation d) hydrostatic stress in adjacent bulk elements, e) interface triaxiality, f) transverse stresses in adjacent bulk elements, g) calculated cavity growth rate: $\dot{V}_{L}, \dot{V}_{H}$ and all their components, f) von Mises stresses in adjacent bulk elements. . . . . . . . . . . . . . . . . . . . . 41

4.10 Cavity growth rates $\dot{V}_{L}$ and $\dot{V}_{H}$ and all their components before failure. . . . 42

4.11 Comparison of global strains and strain rates for two different simulations, one using both volumetric growth rate $\operatorname{terms}\left(\dot{V}_{L}\right.$ a and $\dot{V}_{H}$, squares $)$ and the other using only the $\dot{V}_{L}$ term (solid lines). a) Comparison of the strain rates versus time. The individual contributions of the interface and grain rates to the overall strain rate is also shown. b) Overall strain for both simulations. .

4.12 Mesh refinement study results. . . . . . . . . . . . . . . . .

5.1 Cell average equivalent strain versus time (orange) for uniaxial simulations. The interface equivalent strain (blue) and the grain equivalent inelastic strain (brown) are also depicted. Stress level decreases from left to right from $180 \mathrm{MPa}$ to $60 \mathrm{MPa} \ldots \ldots \ldots \ldots \ldots \ldots \ldots \ldots \ldots$

5.2 RMSRE of effective stress parameters calibrated against uniaxial and biaxial data versus the predicted time to rupture for the different effective stress models. Blue bars represent the RSMRE when only considering biaxial data, orange bars when considering all datapoints. . . . . . . . . . .

5.3 Comparison of RMSRE for effective stress parameters calibrated against: (i) uniaxial and biaxial data (blue) and (ii) all datapoints (orange). The error is computed by using all data points . . . . . . . . . . . . . . .

5.4 Comparison RSMRE distribution for the $\max$ stress of simulated versus predicted time to rupture. Color represent RSMRE error. a) biaxial fit versus biaxial data, b) biaxial fit versus all data c) triaxial fit versus triaxial data .

5.5 Comparison RSMRE distribution for the mises stress of simulated versus predicted time to rupture. Color represent RSMRE error. a) biaxial fit versus biaxial data, b) biaxial fit versus all data c) triaxial fit versus triaxial data .

5.6 Comparison RSMRE distribution for the Hayhurst_1972 stress of simulated versus predicted time to rupture. Color represent RSMRE error. a) biaxial fit versus biaxial data, b) biaxial fit versus all data c) triaxial fit versus triaxial data . . . . . . . . . . . . . . . . . 
Initial study of notch sensitivity of Grade 91 using mechanisms motivated crystal plasticity finite element method

5.7 Comparison RSMRE distribution for HLM_1978 stress of simulated versus predicted time to rupture. Color represent RSMRE error. a) biaxial fit versus biaxial data, b) biaxial fit versus all data c) triaxial fit versus triaxial data .

5.8 Comparison RSMRE distribution for Huddleston stress of simulated versus predicted time to rupture. Color represent RSMRE error. a) biaxial fit versus biaxial data, b) biaxial fit versus all data c) triaxial fit versus triaxial data .

5.9 Comparison RSMRE distribution for the RCC-MRx_mises stress of simulated versus predicted time to rupture. Color represent RSMRE error. a) biaxial fit versus biaxial data, b) biaxial fit versus all data c) triaxial fit versus triaxial data . . . . . . . . . . . . . . . . . .

5.10 Comparison RSMRE distribution for the $R C C$-MRx_max stress of simulated versus predicted time to rupture. Color represent RSMRE error. a) biaxial fit versus biaxial data, b) biaxial fit versus all data c) triaxial fit versus triaxial data . . . . . . . . . . . . . . . . . . . 61 

Initial study of notch sensitivity of Grade 91 using mechanisms motivated crystal plasticity finite element method

September 2019

\section{List of Tables}

4.1 Grain boundary cavitation material parameters. . . . . . . . . . . . . . . 32

4.2 Grain boundary cavitation damage parameters. . . . . . . . . . . . . . . 33

4.3 Grain boundary cavitation SNES parameters. For more details the reader is referred to PESTC manual [6]. . . . . . . . . . . . . . . . . . . . 33

4.4 Grain boundary model options. . . . . . . . . . . . . . . . . . . . . . 33

4.5 Boundary conditions for the 2 element displacement controlled simulations. . 35

4.6 Boundary conditions for the stress controlled, 2 element simulations, Traction values are in $\mathrm{MPa}$. . . . . . . . . . . . . . . . . . . . . 40

4.7 CPFEM simulation parameters. . . . . . . . . . . . . . . . . . 44

4.8 Boundary conditions for the stress controlled, 2 element simulations. Traction

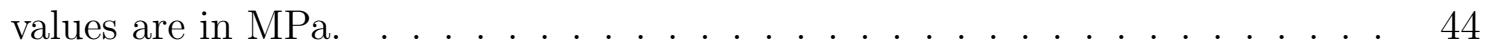

5.1 Stress states imposed for the different simulations classified by stress state. Triaxility and calculated time to rupture are also reported. Horizontal lines group simulations into uniaxial, biaxial and triaxial groups. . . . . . . . 49

5.2 Identified values of $A$ and $n$ for different stress ranges (Eq. 5.10). . . . . . . 51

5.3 List of equations for the analyzed effective stresses. . . . . . . . . . . . . 51

5.4 List of best fit parameters for all effective stresses. . . . . . . . . . . . . . 52 

Initial study of notch sensitivity of Grade 91 using mechanisms motivated crystal plasticity finite element method

September 2019

\section{Introduction}

\subsection{Overview}

The heat pipe microreactor concept involves the use of a solid core block to encapsulate the nuclear fuel and the passive extraction of heat from the core block to the energy conversion system with advanced heat pipes. This results in a compact reactor configuration with minimal moving parts and enables inherent power regulation, autonomous operation, and inherit load following It is well suited for delivering economical and sustainable power to remote locations. Current vendor heat pipe microreactor design targets process heat applications up to $600 \mathrm{C}$ and 10-year reactor core life.

The core block is a key component of the heat pipe microreactor design. It integrates the functions of reactor vessel, core structural components, and fuel cladding of traditional Gen IV advanced reactor designs into a single structure. The current reference material for the core block construction is Type 316 stainless steel. The identification of additional candidate materials for the core block construction would expand the design envelope for higher safety margins and for exploration of more innovative and economical designs.

Grade 91 is a ferritic-martensitic steel that has a lower thermal expansion coefficient and a higher heat conductivity as compared with Type 316 stainless steel, resulting in lower thermal stresses developed in the core block during reactor operations. It also has higher resistance to radiation induced swelling. It does not work harden as much and hence has less challenging machining issues than Type 316 stainless steel.

The core block design involves thousands of holes for the placement of fuel pellets and the insertion of heat pipes. Multiaxial stresses are generated in the ligaments between the holes. Thus it is important to understand the influence of stress multiaxiality on creep rupture life and creep ductility of Grade 91 for the safe, efficient design of the Grade 91 core block.

Typically the design of high temperature components like core blocks is controlled by time-dependent creep properties. For constant loads oftentimes structures are designed to prevent the initiation of creep damage by keeping the stress in the structure below a creep rupture strength. This creep rupture strength expresses the time-dependent relation between the applied stress and temperature and the time-to-failure. Essentially, the creep rupture strength $\sigma_{r}$ for temperature $T$ and time $t$ is the stress that will cause rupture in time $t$ if the material is held at stress $\sigma_{r}$ and temperature $T$.

Nearly universally design creep rupture strengths are developed by correlating and extrapolating uniaxial creep rupture test data. Figure 1.1 shows a typical uniaxial creep specimen. In these types of tests the stress applied to the specimen gauge is uniaxial - there is only one non-zero stress component. Typical design practice is to take data from these types of tests, for example rupture stresses, correlate and extrapolate that data using empirical models, for example the Larson-Miller approach used by the ASME Code [30], and develop design rupture stresses from this extrapolation.

Of course even standard high temperature reactor components seldom experience uniaxial loading. Instead, stress states in structural components are generally multiaxial, i.e. stresses occur along more than one direction. Multiaxial stress states can be parameterized using a decomposition of a generic stress tensor $\boldsymbol{\sigma}$ into principal components. Mathematically, this is an eigen decomposition of the stress tensor into a sum of scalar principal components, 
Initial study of notch sensitivity of Grade 91 using mechanisms motivated crystal plasticity finite element method

September 2019

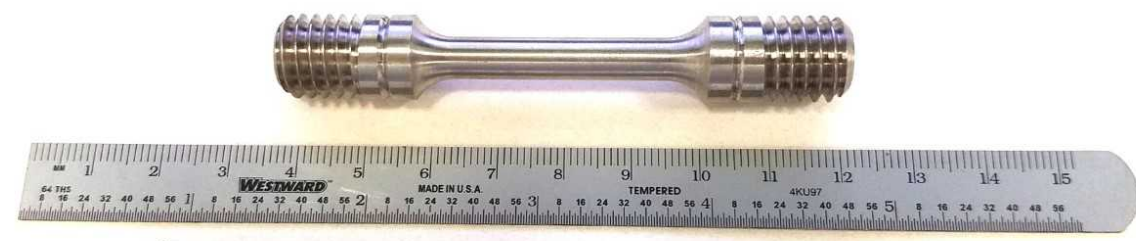

Figure 1.1: Standard uniaxial creep specimen. The smooth transition to the gauge region in the center of the specimen ensures the stress state in the critical region is uniaxial. Photo used with permission from Y. Wang, Oak Ridge National Laboratory.

called principal stresses, acting along orthogonal principal directions:

$$
\boldsymbol{\sigma}=\sum_{i=1}^{3} \sigma_{i}\left(\mathbf{n}_{i} \otimes \mathbf{n}_{i}\right) .
$$

The principal stresses $\sigma_{i}$ are typically ranked from highest to lowest, i.e. $\sigma_{1} \geq \sigma_{2} \geq \sigma_{3}$ with $\sigma_{1}$ called the maximum principal stress. These principal stresses can be used to categorize loading conditions. Loading with $\sigma_{2}=\sigma_{3}=0$ is uniaxial, loading with $\sigma_{3}=0$ is biaxial, and loading with all three nonzero principal stresses is triaxial. Both biaxial and triaxial stress states can be categorized as multiaxial. This report will labels stress states where $\sigma_{1}=\sigma_{2}$ and $\sigma_{3}=0$ equal biaxial and stress states where $\sigma_{1}=\sigma_{2}=\sigma_{3}$ equal triaxial to distinguish these special cases from the general biaxial and triaxial conditions.

High temperature design methods use an effective stress, here notated $\sigma_{e}$, to account for stress multiaxiality. In essence, a particular effective stress measure is a correlation between a multiaxial stress state, often characterized by combinations of the three principal stresses to ensure the correlation is appropriately invariant under arbitrary rotations, to the uniaxial rupture stress. The key idea of an effective stress is to find a mapping from multiaxial stresses to a uniaxial stress so that a material under a multiaxial loading mapping to the effective stress $\sigma_{e}$ fails in the same time as a creep test loaded uniaxially with that same stress $\sigma_{e}$. That is, an effective stress is a way of relating multiaxial stress states to the creep rupture correlation devised using uniaxial creep test data.

In practice, effective stress measures are devised from a small amount of multiaxial creep test data. Commonly only two types of multiaxial creep tests are available: pressurized tubes [25] (Fig. 1.2), sometimes under a combination of pressure and axial stress, and tensiontorsion tests $[13,21,48]$. Both of these test types provide only biaxial load. Effective stress measures are devised and calibrated using this biaxial creep rupture data.

For standard high temperature reactor designs only having biaxial test data is not a significant drawback. Typically, many components in standard high temperature designs can be approximated as thin-walled pressure vessels with nearly biaxial stress states. Of course some components will experience triaxial stresses, nozzles being the classical example, but at least historically the majority of the design can be performed using biaxial stress analysis. 
Initial study of notch sensitivity of Grade 91 using mechanisms motivated crystal plasticity finite element method

September 2019

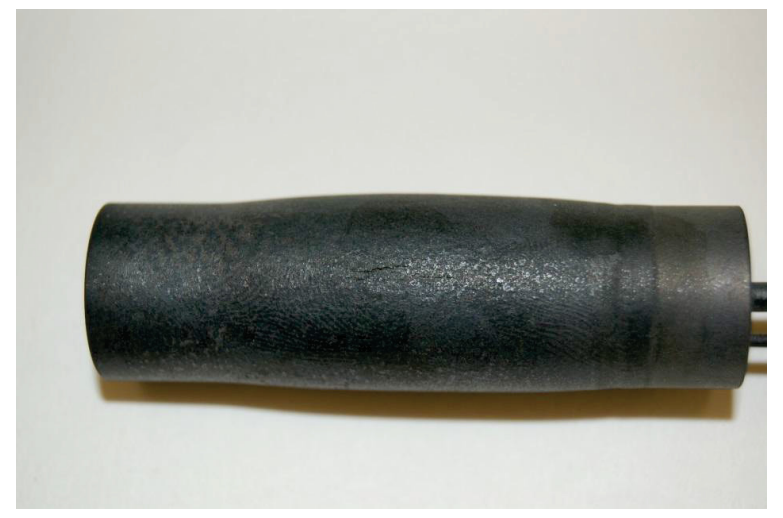

Figure 1.2: Typical pressurized creep specimen tested to a failure. The thin-walled capped tube is pressurized which induces a biaxial stress state in tubular shell. The principal stresses are, approximately, $\sigma_{1}=2 \sigma_{2}$ with $\sigma_{3}=0$. Figure reproduced from [52].

Core blocks of heat pipe microreactors cannot be reasonably approximated as pressure vessels under biaxial load. Core block structures will experience highly multiaxial stress states and so an accurate, effective method for multiaxial creep design will be crucial to the success of core block designs. In line with standard design practice, this report then attempts to find an accurate effective stress relation for the design of components experiencing multiaxial loading. This report focuses on the ferritic-martensitic Grade 91 steel and a temperature of $T=600^{\circ} \mathrm{C}$.

However, there is no triaxial experimental test data to evaluate existing effective stress relations or develop new, more accurate expressions. Instead, this report uses a physicallybased crystal plasticity finite element method (CPFEM) model for creep rupture in Grade 91 steel to explore effective stress relations. This CPFEM model has been described in previous DOE reports and journal publications focusing on the development of the model, the model results for uniaxial loading $[34,38]$, and the application of the model to multiaxial loading in the context of notch strengthening and weakening effects [36,37]. These results described in these previous publications demonstrate that the Grade 91 CPFEM model accurately recovers the uniaxial creep test data for temperatures up to $600^{\circ} \mathrm{C}$ and argues that the physically-based model can more reliably extend short-term creep test data to longer design lives, compared to empirical extrapolation methods. The work on notch effects demonstrates the model makes reasonable predictions for multiaxial creep, though as described here only limited validation data for these conditions is available.

Notch effects are a simple way to characterize the effect of stress multiaxiality on creep rupture. A notched creep test and an equivalent standard specimen are tested under the same conditions to see which fails first - the notched or unnotched specimen. If the notched specimen fails first the material is notch weakening for that particular combination of load, temperature, and notch geometry whereas if the smooth specimen fails first the material is notch strengthening. The notch generates stress multiaxiality and so this type of test crudely assess the effect of multiaxial stress states on rupture. The challenge with notched creep tests is that the stress state in the critical region is unknown, uncontrolled, and changes with time. Therefore, these type of tests are unsuitable for devising an effective stress measure to use in design. 
Initial study of notch sensitivity of Grade 91 using mechanisms motivated crystal plasticity finite element method

September 2019

This report describes a method for using the physically-based CPFEM model for creep in Grade 91 to test and develop effective stress measures for the design of core blocks in heat pipe microreactors. Unlike previous work on notch sensitivity the goal here is to provide designers a quantifiable way to design structures experiencing triaxial stresses against creep rupture by developing an accurate effective stress measure. The key point is that while triaxial creep tests are impossible, or at least have not yet been developed, the physically based model can be loaded in arbitrary stress states. The model can therefore be used to generate synthetic test data for use in developing or assessing effective stresses under realistic triaxial stress states.

\subsection{Brief survey of multiaxial creep}

This section gives a brief overview of previous work on representing creep failure under multiaxial load. Chapter 5 discusses a set of particular effective stresses commonly used in design and evaluated in this report, this section provides a general overview of multiaxial effects.

The earliest literature on multiaxiality effects on creep and creep rupture focus on notch effects $[8,11,15,45]$. Some of the results described in these early 1950s papers date back to World War II era research on aerospace materials. For a more detailed summary see [36]. Most of the work at this early data is experimental and qualitative, trying to understand which characteristics (stress state, notch geometry, material parameters) affect notch sensitivity.

Hayhurst, Leckie, and colleagues initiated more quantitative work on the effect of multiaxiality through their development of continuum damage mechanics for modeling and predicting creep failure in components (c.f. [19, 20, 22, 31]). There some difference between their work on continuum damage mechanics and traditional engineering design approaches, but both methods account for multiaxial effects in a similar manner through the use of a scalar effective stress. This report considers two particular effective stresses originally proposed by the Hayhurst-Leckie school in Chapter 5.

Huddleston [24-26] developed a second widely-used family of effective stresses, referred to here as the Huddleston model. His method of correlating multiaxial stresses to uniaxial creep rupture data is currently in use in Section III, Division 5 of the ASME Boiler and Pressure Vessel Code covering the design of high temperature reactor components [2].

Both of the aforementioned methods for correlating multiaxial creep to uniaxial data are more-or-less empirical and based on direct biaxial creep testing. More modern approaches attempt to tie the problem of creep failure to void growth in ductile materials. The CPFEM interface model described in Chapter 4 falls into this school of thought, albeit in the context of a micromechanical model. Macroscale models tend to follow the work of Rice and Tracey [42], Cocks and Ashby [9], Gurson [18], Needleman [39], and Tvergaard [50]. [54] contains a good summary of recent developments along these lines.

Finally, mention should be made of the current British approach exemplified in the R5 standard for fitness for service evaluations at elevated temperatures [14]. While the standard does retain an allowable stress approach using the Huddleston stress to relate multiaxial stress states back to a scalar allowable stress, the main approach used in R5 is ductility exhaustion. Whereas up until now this report discusses a creep rupture stress and the 
Initial study of notch sensitivity of Grade 91 using mechanisms motivated crystal plasticity finite element method

September 2019

concept of an effective stress relating multiaxial to uniaxial rupture failures, the ductility exhaustion method hypothesizes that creep ductility is the key parameter determining failure [17]. Essentially the method ties creep failure to a critical amount of accumulated inelastic strain (the creep ductility), which depends on temperature and the strain rate of the applied loading. Applying the ductility exhaustion approach to multiaxial loading then requires an effective strain measure which relates a multiaxial state of strain back to the axial strain, correlated to a creep ductility database populated from the results of uniaxial creep tests. The R5 standard uses an effective strain developed by Spindler [47], which however in the end refers to the stress state of the material rather than to the strains directly. While the ductility exhaustion approach is a valid alternative to stress-time formulations, this report describes creep failure in terms of stresses to maintain compatibility with the standard U.S. high temperature design methods described in the ASME Boiler and Pressure Vessel Code.

\subsection{Organization of this report}

Chapter 2 describes a finite element analysis of a representative core block in a heat pipe microreactor design in order to quantify the triaxial stress states found in the web of core blocks. The goal of this chapter is to assess the range of triaxial stress states that may be found in future core block microreactors and compare these stress states to those found in more typical high temperature reactor designs where the stresses can often be approximated as biaxial. Chapters 3 and 4 describe improvements made to the CPFEM model since the last report on this topic ([37]). Chapter 3 covers the single crystal constitutive model and gives a general overview of CPFEM modeling while Chapter 4 covers the interface-cohesive model used to represent creep damage and failure on grain boundaries (GBs). The goal of these improvements is to enable the model to reach realistic failure strains and rupture lives. Previous incarnations of the model were limited by numerical issues to times only slightly past the initiation of tertiary creep. The new, improved model can continue all the way to complete material failure. A secondary objective was to transfer the model from the WARP3D finite element code [29] to the MOOSE framework [16] developed and maintained by Idaho National Laboratory to facilitate future extensions of the modeling framework to multiphysics simulations.

Chapter 5 describes the main results of this report. This chapter describes a modeling framework used to quantify the effect of triaxial stresses on the creep life of Grade 91, assesses several different effective stresses, and recommends an effective stress for the design of core blocks in heat pipe microreactors and other elevated temperature structural components that experience highly-triaxial stresses. Finally, Chapter 6 summarizes the key conclusions of this work and discusses the implications on high temperature design in general. 

Initial study of notch sensitivity of Grade 91 using mechanisms motivated crystal plasticity finite element method

September 2019

\section{Assessing the multiaxial stress state in a core block design}

To demonstrate the complex stress states that will occur in core blocks of heat pipe microreactors this chapter summarizes the results of a preliminary inelastic stress analysis of a core block under a constant thermal load. Full details of the preliminary analysis are provided in an analysis report attached as Appendix A. The material analyzed here is $316 \mathrm{H}$ stainless steel, but character of the stress state, i.e. biaxial versus triaxial, would not greatly change for a Grade 91 design.

The design geometry and loading conditions were extracted from [33, 49]. The loading considered was a single heat up to normal operating power with approximate thermal boundary conditions for the fuel pin heat generation and heat pipe heat removal. The reactor was brought to the operating power and held in that condition for a design life of 100,000 hours. The analysis did not consider pressure on the fuel or heat pipe holes as the primary stresses induced by these pressures are expected to be small compared to the thermal stresses. More importantly, the analysis did not consider the effect of load cycling. In an actual microreactor design, creep-fatigue damage by cycling caused by reactor power down/power up events will control the design life of the core block. Each cycle will reset the creep stress relaxation history of a point to the high stresses typical of the initial elastic stress in the current calculation, therefore greatly increasing damage in the material when compared to the current steady-state analysis. The "load and hold" load history applied in the current analysis is therefore not representative of actual reactor service loads. However, the primary purpose of the analysis is to estimate the stress states experienced by core block webs in a typical core block design.

Figure 2.1 shows the finite element mesh used in the calculation. This represents a $60^{\circ}$ sector of a full core block design. Thermal boundary conditions in the hole regions represent either the heat generation of an active fuel pin or the heat removal of a functional heat pipe.

The analysis uses a simple inelastic constitutive model to represent the creep deformation and corresponding stress redistribution in the $316 \mathrm{H}$ core block.

The mechanical, and to a lesser degree, thermal boundary conditions on the sides of the block section are not well modeled in the current analysis and so results from the region of the core block near these boundaries should be ignored. Additionally, global bending stresses

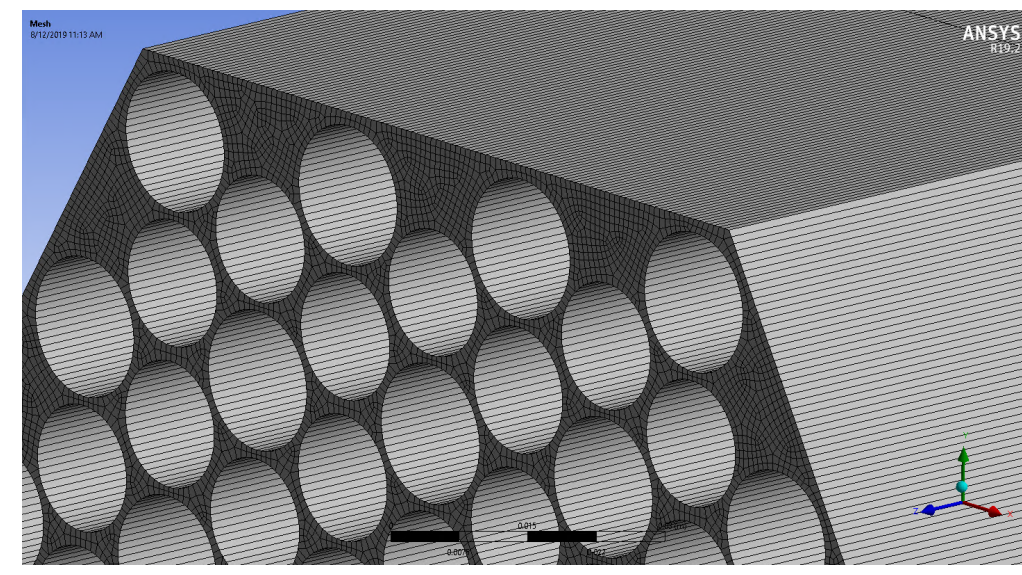

Figure 2.1: Finite element mesh used in the calculation. 
Initial study of notch sensitivity of Grade 91 using mechanisms motivated crystal plasticity finite element method

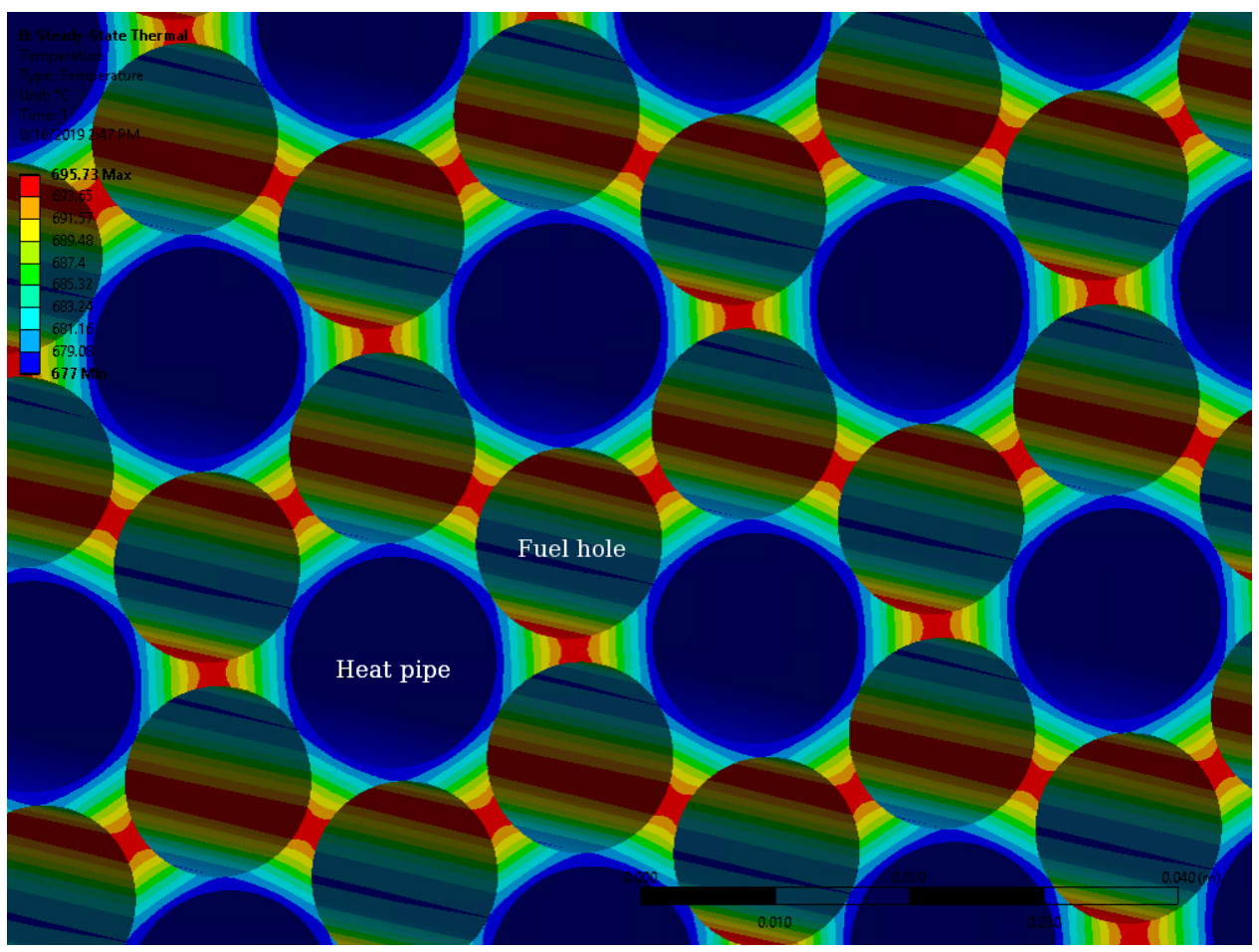

Figure 2.2: Representative temperature field near the center of the modeled region.

introduced by core block supports were not included in the analysis.

Figure 2.2 shows a representative temperature distribution away from the edges of the modeled region. A strong thermal gradient developed along the web between heat pipes, inducing thermal stresses in the core block. Creep will cause these thermal stresses to relax as time progresses, though transient cycles, not modeled here, would cause the thermal stresses to reset to higher values as each transient occurs.

Figure 2.3 shows the triaxiality factor

$$
\mathrm{TF}=\frac{\sigma_{1}+\sigma_{2}+\sigma_{3}}{\sqrt{\frac{\left(\sigma_{1}-\sigma_{2}\right)^{2}+\left(\sigma_{2}-\sigma_{3}\right)^{2}+\left(\sigma_{3}-\sigma_{1}\right)^{2}}{2}}}
$$

where $\sigma_{1}, \sigma_{2}$, and $\sigma_{3}$ are the principal stresses, near the start of the hold period, where the triaxiality tends to be the highest. Moderate triaxial stresses develop near the heat pipes in the center of the modeled sector, with values of triaxiality increasing towards the edges of the model. Ignoring the region immediately adjacent to the model edges, where the inaccurate boundary conditions significantly affect the stress field, the maximum value of triaxiality is greater than 2.0. With the definition of triaxiality given in Eq. 2.1 a value of $\mathrm{TF}=1$ indicates uniaxial tension loading. Therefore, the core block analysis indicates a moderately high degree of triaxiality might be expected. For reference, the triaxiality factor for a biaxial, thin walled, capped pressure vessel away from discontinuities is $\sqrt{3} \approx 1.73$. Additionally, the stress states are substantially more multiaxial and vary in the plane of the core block significantly more than a standard thin-walled pressure vessel.

The triaxial, 3D stress states in the core block do not typically occur in standard reactor components that can be modeled as thin-walled pressure vessels. As such, high temperature 
Initial study of notch sensitivity of Grade 91 using mechanisms motivated crystal plasticity finite element method

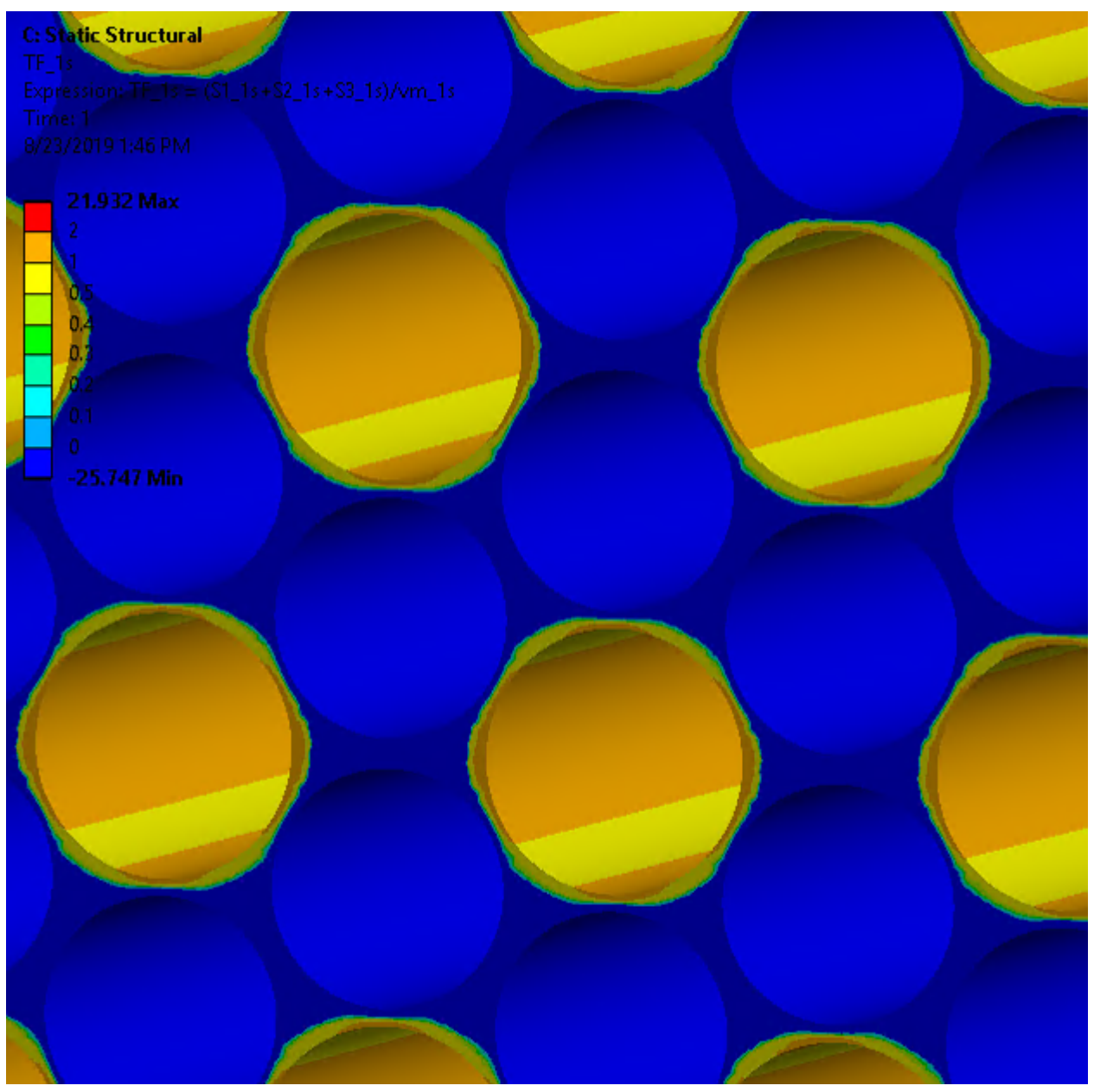

Figure 2.3: Representative triaxiality factors (Eq. 2.1) near the center of the modeled region. 
Initial study of notch sensitivity of Grade 91 using mechanisms motivated crystal plasticity finite element method

September 2019

structural design practice may need to be improved to accurately represent creep and creepfatigue damage under highly triaxial conditions. 
Initial study of notch sensitivity of Grade 91 using mechanisms motivated crystal plasticity finite element method

September 2019

\section{Improvements to the grain bulk model}

As described in the introduction, the CPFEM model for creep deformation and rupture of Grade 91 was ported to the MOOSE [16] finite element framework in order to improve the numerics of the model so that it can capture true rupture strains and times and to provide future extensibility. In order to accomplish the transition, ANL implemented three additions to the MOOSE framework:

1. Developed an interface-cohesive model and ported the GB damage model into the new MOOSE interface framework (discussed in Chapter 4).

2. Implemented a new grain bulk single crystal constitutive model framework in NEML [3], which already has an interface to MOOSE (discussed here).

3. Improved the MOOSE large deformation tensor mechanics module to achieve better convergence for CPFEM simulations (discussed here).

This chapter also provides a brief overview on how CPFEM simulations work and the advantages and disadvantages of the technique. For a more complete description of the particular CPFEM model for creep used in this work see the previous research reports $[34,37]$.

\subsection{Brief overview of CPFEM}

The crystal plasticity finite element method is a way to simulate the mesoscale deformation processes causing inelastic deformation and damage in metal polycrystals [44]. The methodology represents these deformation and damage mechanisms at the mesoscale where the response of the material can still reasonably be represented with a continuum field theory. However, the underlying constitutive equations represent microscale deformation and damage mechanisms, rather than empirical descriptions of deformation and damage as are often used in macroscale engineering simulations.

Fundamentally, CPFEM calculations solve the same field equations as a standard large deformation engineering solid mechanics calculation. The fundamental field equation is the balance of linear momentum in the deformed configuration, here presented in a quasistatic form without body forces:

$$
\begin{array}{llll}
\nabla_{\mathbf{x}} \cdot \boldsymbol{\sigma} & =\mathbf{0} & \text { on } & \Omega \\
\boldsymbol{\sigma} \cdot \mathbf{n} & =\hat{\mathbf{t}} & \text { on } & \partial \Omega_{n} \\
\mathbf{u} & =\hat{\mathbf{u}} & \text { on } & \partial \Omega_{e}
\end{array}
$$

where $\boldsymbol{\sigma}$ is the Cauchy stress, $\Omega$ represents the deformed domain, $\mathbf{n}$ is the outward pointing surface normal, $\partial \Omega_{n}$ is the part of the boundary of $\Omega$ over which natural boundary conditions are applied, $\hat{\mathbf{t}}$ are the imposed tractions, $\mathbf{u}$ is the unknown displacement field to be determined, $\partial \Omega_{e}$ is the part of the boundary of $\Omega$ over which essential boundary conditions are applied, and $\hat{\mathbf{u}}$ are the imposed displacements. $\nabla_{\mathbf{x}}$ denotes the divergence in the current coordinates, over the domain $\Omega$ 
Initial study of notch sensitivity of Grade 91 using mechanisms motivated crystal plasticity finite element method

September 2019

The unknown field in this formulation is $\mathbf{u}$ but only stress appears in the equilibrium equation. The closure relation is a function mapping a displacement (actually the gradient of the displacement) at a material point to the corresponding stress. This functional relation has two parts: (1) kinematics defining the appropriate measure of deformation and the map between the material frame of reference and the current configuration used in the equilibrium calculation and (2) constitutive relations relating this deformation measure to the stress. The formulation described here, and its corresponding implementation in NEML, uses modular constitutive relations, so that any single crystal constitutive model can be easily implemented in the kinematic framework described below. Because NEML already ties into MOOSE, providing the solid mechanics constitutive relations in a simulation, these single crystal constitutive models can easily be used in MOOSE without additional effort.

Note that in this description of the problem a single crystal CPFEM constitutive model is exactly analogous to a standard material model embedded in a solid mechanics finite element solver. The only difference is the choice of kinematic framework for describing deformation and the interpretation of the constitutive model. For a standard macroscale finite element calculation the model, often empirically, describes the macroscale deformation of the material, tactically homogenizing the response of many single crystal grains for a metal, whereas for CPFEM the constitutive model describes the response of a single crystal. This is fundamentally both the strength and the weakness of CPFEM approaches. It means that a CPFEM model can be implemented in any standard finite element framework simply by embedding a particular material model. However, it also means that the resolution of the CPFEM model cannot descend to scales on which the fundamental assumption of describing deformation with a continuous field equation breaks down.

To give the reader some idea of what CPFEM calculations look like, Figure 3.1 visualizes the initial mesh and the initial grain orientations (a), the final grain orientations (b), and a result field (c), here the von Mises effective stress, from a sample calculation. As the figure demonstrates, a fully-resolved CPFEM simulation discretizes each initially single crystal grain with multiple finite elements. The method is then capable of subgrain resolution, with the limits on scale discussed above. This means in later calculations the CPFEM simulations of creep are capable of resolving cavitation on critical grain boundaries, accounting for the deformation and stress in neighboring subgrain regions. Figure 3.1(a) demonstrates the initialization of CPFEM simulations. In addition to internal variables describing microstructural evolution and damage, discussed below, a CPFEM model tracks the crystallographic orientation of each material point. Typically, unless grain misorientation data is available, each grain will have a single crystal orientation, as shown in (a). The crystal orientation of each point is essentially an internal variable for the constitutive model, evolved with an evolution equation discussed below. Figure 3.1(b) shows that CPFEM calculations track the evolution of grain orientation throughout the simulation. Grains both reorient as a whole and develop misorientation, starting from the initially perfect single crystal orientation. CPFEM calculations can then be used to model the evolution of texture in a material. Finally, Figure 3.1(c) visualizes the von Mises effective stress over the microstructure at an arbitrary point in the deformation history. CPFEM makes subgrain resolution stress and deformation information available to compare to experimental measurements and to drive the evolution of internal variables representing the development of the microstructure and the initiation and growth of damage. Chapters 4 and 5 describe how to use a CPFEM framework to model 
Initial study of notch sensitivity of Grade 91 using mechanisms motivated crystal plasticity finite element method

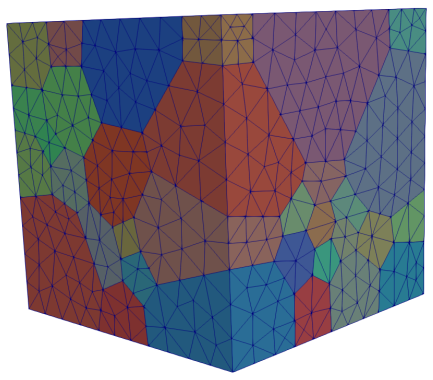

(a)

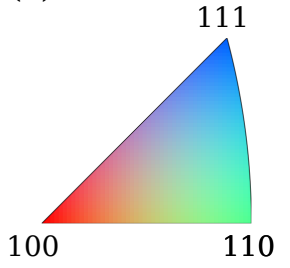

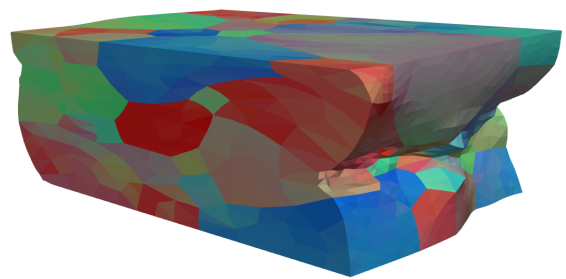

(b)

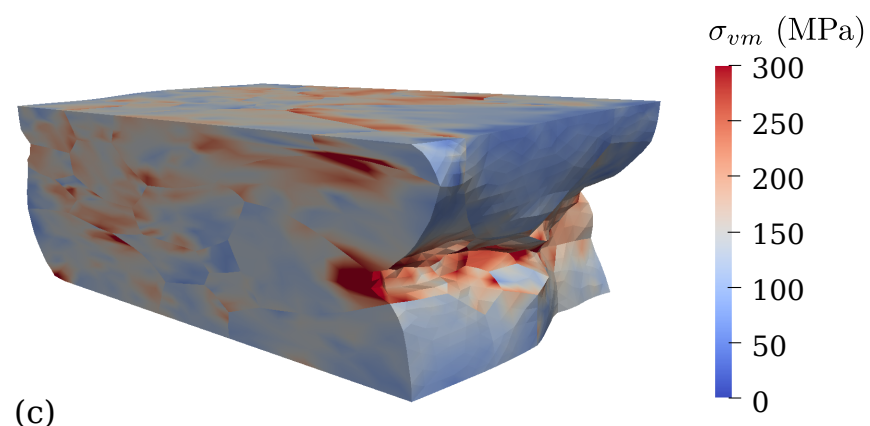

Figure 3.1: Example CPFEM analysis of a cube of FCC material undergoing $50 \%$ rolling strain. (a) Initial grain orientations and the problem mesh. (b) Final grain orientations. (c) von Mises stress field from the final simulation step.

the evolution of creep-induced cavitation on GBs and use a complete CPFEM simulation to model creep rupture in Grade 91 steel.

\subsection{Model implemented in NEML/MOOSE}

\subsubsection{Kinematics}

Fundamentally, crystal plasticity kinematics derives from the multiplicative decomposition of the deformation gradient into elastic and plastic components

$$
\mathbf{F}=\mathbf{I}+\nabla_{\mathbf{X}} \mathbf{u}=\mathbf{F}^{p} \cdot \mathbf{F}^{e}
$$

where $\nabla_{\mathbf{X}}$ denotes the divergence in the reference coordinates over the domain $\Omega_{0}$. The subsequent derivation, starting from this fundamental hypothesis, was then developed by Rice [43], Hill and Rice [23], and Asaro and Rice [5] into the standard crystal plasticity kinematics. An early work by Asaro [4] provides a good general overview.

Expanding the multiplicative decomposition to find the spatial velocity gradient yields

$$
\mathbf{l}=\dot{\mathbf{F}} \mathbf{F}^{-1}=\dot{\mathbf{F}}^{e} \mathbf{F}^{e-1}+\mathbf{F}^{e} \dot{\mathbf{F}}^{p} \mathbf{F}^{p-1} \mathbf{F}^{e-1}
$$

Label

$$
\overline{\mathbf{l}}^{p}=\dot{\mathbf{F}}^{p} \mathbf{F}^{p-1}
$$




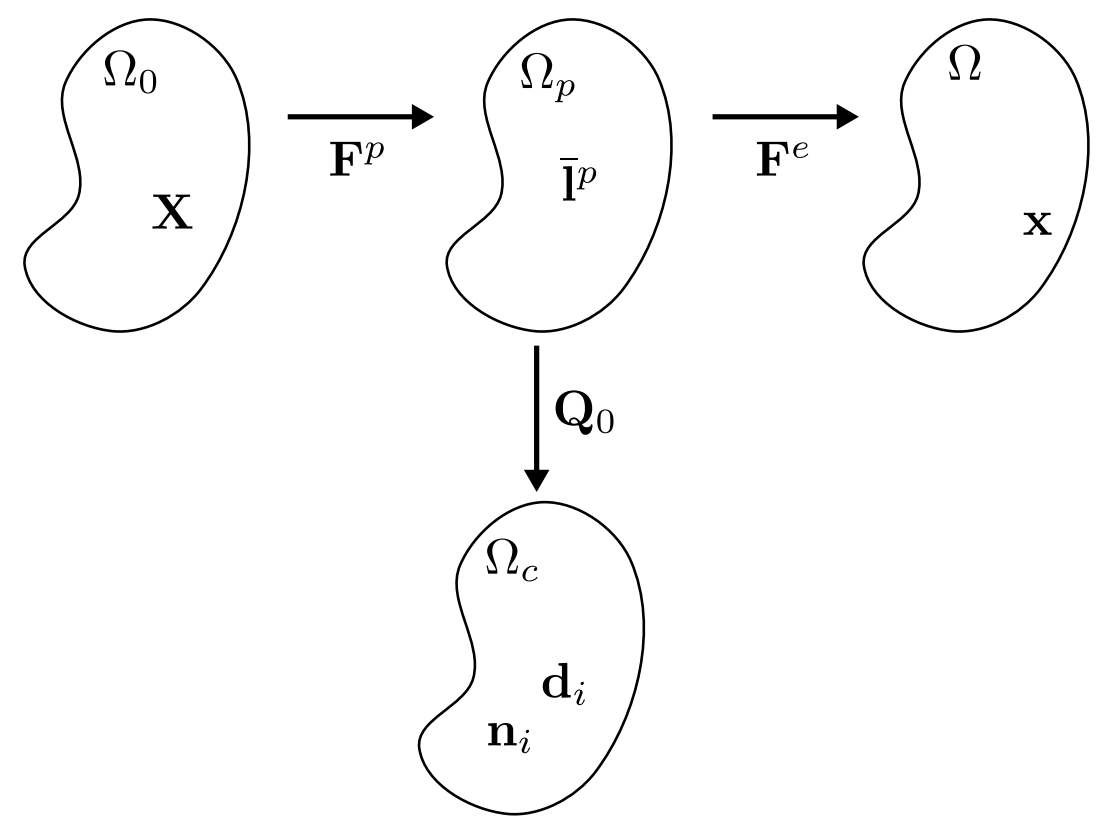

Figure 3.2: Description of the frames of reference in the standard crystal plasticity kinematics.

which reduces Eq. 3.5 to

$$
\mathbf{l}=\dot{\mathbf{F}}^{e} \mathbf{F}^{e-1}+\mathbf{F}^{e} \overline{\mathbf{l}}^{p} \mathbf{F}^{e-1}
$$

The fundamental constitutive assumption of crystal plasticity is that

$$
\overline{\mathbf{l}}^{p}=\sum_{i=1}^{n_{\text {slip }}} \dot{\gamma}_{i}\left(\mathbf{Q}_{0} \cdot \mathbf{d}_{i} \otimes \mathbf{n}_{i} \cdot \mathbf{Q}_{0}^{T}\right)
$$

where $\dot{\gamma}_{i}$ is the slip rate along the slip system defined by direction $\mathbf{d}_{i}$ and normal $\mathbf{n}_{i}$, often taken to be a function of the stress $\boldsymbol{\sigma}$ and some set of internal variable describing the microstructure of the material, $\mathbf{h}$ (see below). The rotation $\mathbf{Q}_{0}$ takes the initial reference frame to the crystal frame of reference. This rotation is what would be measured in a classical diffraction experiment. Figure 3.2 shows the configurations implied by this decomposition and constitutive assumption. Note that the lattice frame of reference is attached by the constant rotation $\mathbf{Q}_{0}^{T}$ to the unloaded intermediate frame $\Omega_{p}$, not to the original reference frame $\Omega_{0}$. Asaro and coworkers justify this assumption, which might be called the fundamental assumption of crystal plasticity kinematics, by noting that plastic slip does not disturb the atomic lattice.

Starting from this derivation, which is common to nearly all theories of single crystal plasticity, the implementation in NEML makes several simplifying assumptions. These assumptions are detailed in [35]. The first assumption is that the elastic stretch, but not the elastic rotation, is small, represented as

$$
\mathbf{F}^{e}=(\mathbf{I}+\varepsilon) \mathbf{R}^{e}
$$


so that

$$
\varepsilon \ll \mathbf{I}
$$

and

$$
(\mathbf{I}+\varepsilon)^{-1} \approx \mathbf{I}-\varepsilon
$$

This reduces Eq. 3.7 to

$$
\mathrm{l}=\dot{\varepsilon}-\dot{\varepsilon} \varepsilon+\Omega^{e}-\Omega^{e} \varepsilon+\varepsilon \Omega^{e}-\varepsilon \Omega^{e} \varepsilon+\mathrm{l}_{p}-\mathrm{l}_{p} \varepsilon+\varepsilon \mathrm{l}_{p}-\varepsilon \mathrm{l}_{p} \varepsilon
$$

with

$$
\Omega^{e}=\dot{\mathbf{R}}^{e} \mathbf{R}^{e T}
$$

and

$$
\mathbf{l}_{p}=\mathbf{R}^{e} \overline{\mathbf{l}}_{p} \mathbf{R}^{e T}
$$

Note from Fig. 3.2 and the preceding derivation that the rotation from the current configuration back to the crystal configuration (i.e. what would be measured in a diffraction experiment after deformation) is

$$
\mathbf{Q}=\mathbf{R}^{e} \mathbf{Q}_{0}
$$

and therefore

$$
\dot{\mathbf{Q}} \mathbf{Q}^{T}=\dot{\mathbf{R}}^{e} \mathbf{Q}_{0} \mathbf{Q}_{0}^{T} \mathbf{R}^{e T}=\dot{\mathbf{R}}^{e} \mathbf{R}^{e T}=\mathbf{\Omega}^{e}
$$

Substituting back into 3.12 produces

$$
\mathrm{l}=\dot{\varepsilon}-\dot{\varepsilon} \varepsilon+\Omega^{e}-\Omega^{e} \varepsilon+\varepsilon \Omega^{e}+\mathrm{l}_{p}-\mathrm{l}_{p} \varepsilon+\varepsilon \mathrm{l}_{p} .
$$

Expanding this expression into symmetric and skew parts and dropping terms that are quadratic in $\varepsilon$ and mixed terms like $\varepsilon \dot{\varepsilon}$ on the basis that the elastic deformation is small produces

$$
\begin{gathered}
\mathbf{d}=\frac{1}{2}\left(\mathbf{l}+\mathbf{l}^{T}\right)=\dot{\varepsilon}+\mathbf{d}_{p}+\varepsilon\left(\mathbf{\Omega}^{e}+\mathbf{w}_{p}\right)-\left(\Omega^{e}+\mathbf{w}_{p}\right) \varepsilon \\
\mathbf{w}=\frac{1}{2}\left(\mathbf{l}-\mathbf{l}^{T}\right)=\Omega^{e}+\mathbf{w}_{p}+\varepsilon \mathbf{d}_{p}-\mathbf{d}_{p} \varepsilon .
\end{gathered}
$$

Denote

$$
\Omega^{\star}=\Omega^{e}+\mathbf{w}_{p}
$$

and make the hypoelastic assumption that

$$
\dot{\boldsymbol{\sigma}}=\mathbf{C}: \dot{\varepsilon}
$$

Apply this definition and assumption to Eq. 3.18 and solving for the rate of Cauchy stress gives

$$
\dot{\boldsymbol{\sigma}}=\mathbf{C}:\left(\mathbf{d}-\mathbf{d}_{p}-\mathbf{S}: \sigma \cdot \mathbf{\Omega}^{\star}+\mathbf{\Omega}^{\star} \cdot \mathbf{S}: \sigma\right)
$$

where $\mathbf{S}=\mathbf{C}^{-1}$.

Rearranging Eq. 3.19 to solve for $\boldsymbol{\Omega}^{e}$ gives

$$
\Omega^{e}=\mathbf{w}-\mathbf{w}_{p}-\varepsilon \mathbf{d}_{p}+\mathbf{d}_{p} \varepsilon
$$


Initial study of notch sensitivity of Grade 91 using mechanisms motivated crystal plasticity finite element method

September 2019

A time integration of Eq. 3.22 provides an evolution equation for $\boldsymbol{\sigma}$. A similar time integration of Eq. 3.23 provides an evolution equation for the crystal orientation Q. To form a complete, solvable system of ordinary differential equations these two equations must be supplemented by the constitutive model for $\dot{\gamma}_{i}$ and the evolution equations for any accompanying internal variables describing the material microstructure. The implementation in NEML uses a coupled, backward Euler integration to update the stress and the internal history variables. After successfully updating these variables the implementation then separately updates the crystal orientations, uncoupled to the stress update, using an Euler exponential form to ensure $\mathbf{Q}$ remains in the special orthogonal group.

\subsubsection{Constitutive models}

The single crystal constitutive model framework embedded in NEML defines modular constitutive models so that new models can be easily added for testing and development. Fundamentally, a NEML single crystal model provides the slip rate

$$
\dot{\gamma}_{i}(\boldsymbol{\sigma}, \mathbf{h})
$$

as a function of the stress $\boldsymbol{\sigma}$ and some set of internal variables $\mathbf{h}$. Additionally then the model must also provide the evolution equations for these internal variables, i.e.

$$
\dot{\mathbf{h}}(\boldsymbol{\sigma}, \mathbf{h}) \text {. }
$$

While users are free to implement slip rate models in this generic form, the implementation provides a more conventional interface (i.e. a "Schmid" material) with the form

$$
\dot{\gamma}_{i}\left(\tau_{i}, \bar{\tau}_{i}(\mathbf{h})\right)
$$

where $\tau_{i}$ is the resolved shear on slip system $i$, i.e.

$$
\tau_{i}=\boldsymbol{\sigma}:\left(\mathbf{R}^{e} \boldsymbol{d}_{i} \otimes \mathbf{n}_{i} \mathbf{R}^{e T}\right)
$$

and $\bar{\tau}_{i}$ is the slip system strength, defined as a map from the generic set of history variables h to the strength $\bar{\tau}_{i}$.

While this description is abstract, this definition covers the vast majority of single crystal constitutive models described in the literature. As an example consider the Voce model using a single strength to represent all systems in the crystal. This model has a single history variable $\hat{\tau}$ described by the evolution equation

$$
\dot{\bar{\tau}}=\delta\left(\tau_{s a t}-\bar{\tau}\right) \sum\left|\dot{\gamma}_{i}\right|
$$

with $\delta$ and $\tau_{\text {sat }}$ parameters. The map between the slip system strengths and this internal variable is just the identity, i.e.

$$
\bar{\tau}_{i}=\hat{\tau} .
$$

These expressions, combined with the standard power law model for the slip rate

$$
\dot{\gamma}_{i}=\dot{\gamma}_{i}^{(0)} \frac{\tau_{i}}{\bar{\tau}_{i}}\left|\frac{\tau_{i}}{\bar{\tau}_{i}}\right|^{n-1}
$$


Initial study of notch sensitivity of Grade 91 using mechanisms motivated crystal plasticity finite element method
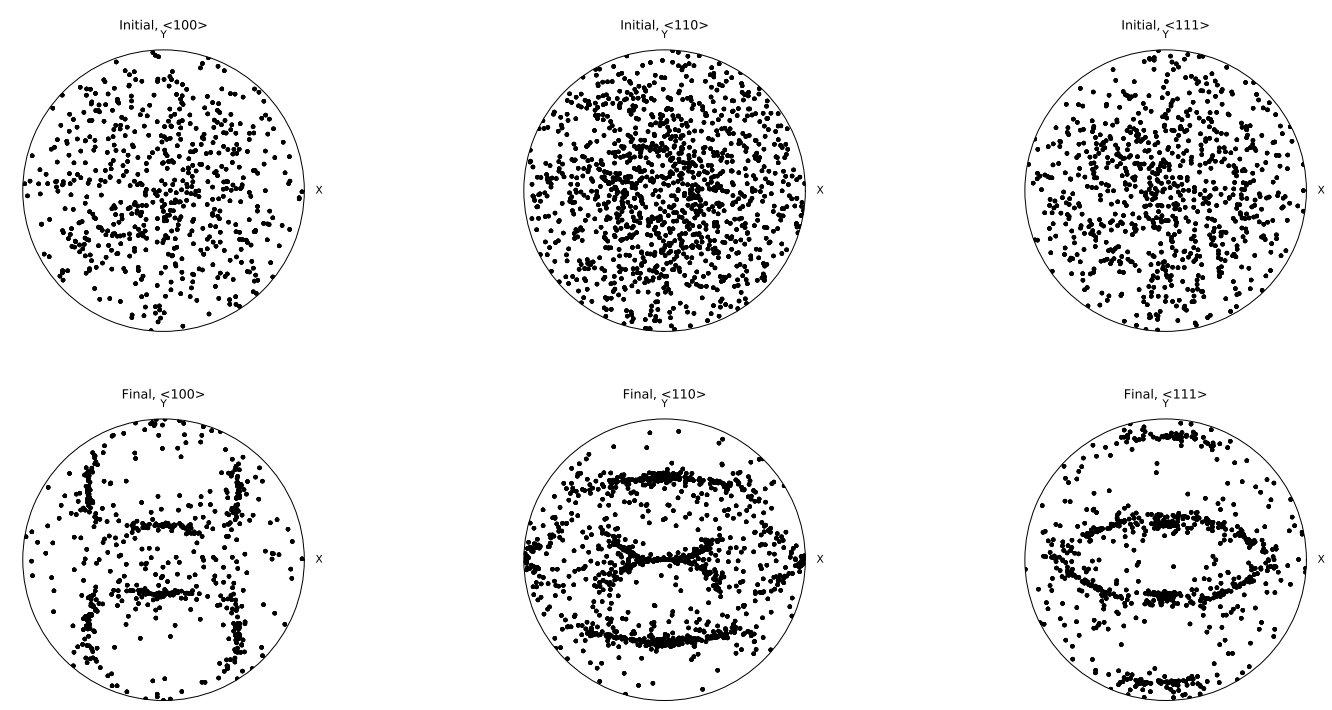

Figure 3.3: Validation test applying 100\% rolling deformation to an FCC polycrystal with an initially random texture. For each column of figures the top figure shows the initial texture and the bottom figure shows the texture after deformation.

with $\dot{\gamma}_{i}^{(0)}$ a parameter, describe a complete single crystal constitutive law. This is the model used in the subsequent simulations of creep in Grade 91. While the Voce hardening model is empirical it can be given a physical interpretation, as described in [28].

In addition to the mathematical implementation of these equations and associated derivatives, integration, and solver routines, the NEML crystal framework provides helper routines for defining lattices based on crystal symmetry groups, postprocessing, and visualizing results of CPFEM calculations. The models can be used in MOOSE just like standard NEML constitutive models, taking advantage of the existing connection between NEML and the MOOSE framework originally developed for modeling macroscale inelasticity in engineering scale component simulations. In addition to the CPFEM interface to finite element solvers, NEML contains python drivers that can be used to simulate homogenized polycrystals. Currently the only homogenization option is the Taylor model.

\subsubsection{Validation}

A common test for a crystal plasticity model is to check that it can reproduce the standard FCC rolling texture. Figure 3.3 plots $\langle 111\rangle,\langle 110\rangle$, and $\langle 100\rangle$ pole figures for an FCC polycrystal before and after $100 \%$ rolling strain (i.e. $100 \%$ plane strain compression). The simulation uses the NEML Taylor model homogenization to simulate the response of a 200 grain polycrystal. The grain orientations are initially random and the slip strength is given by the Voce/power law slip system model described in the previous subsection.

The figure shows that the crystal model reproduces the expected FCC rolling texture. This test integrates several features of the constitutive model — slip system deformation, hardening evolution, and the rotation update - and so serves as an excellent validation test for the framework as a whole. 
Initial study of notch sensitivity of Grade 91 using mechanisms motivated crystal plasticity finite element method

September 2019

\subsection{Modifications to MOOSE for large deformations}

In addition to implementing a single crystal constitutive model some corrections to the MOOSE solid mechanics module (called tensor mechanics) were needed to achieve good convergence in large, parallel CPFEM simulations. The current baseline version of MOOSE does not correctly implement the exact algorithmic tangent for large deformations simulations, meaning these simulations do not achieve optimal, quadratic convergence when using Newton's method to solve the nonlinear force balance equation. As crystal plasticity calculations make little sense except in a large deformation framework, this limitation means the CPFEM simulations take many more steps to converge or fail to convergence entirely, requiring much more compute and wall time to complete a particular calculation. Note that MOOSE by default uses preconditioned Jacobian-free Newton-Krylov methods to solve nonlinear balance equations, where having the exact algorithmic tangent is less important. However, these PJFNK methods have a reduced convergence rate than Newton's methods to begin with and there is no advantage to applying PJFNK solvers, compared to Newton's method, for single-physics problems running on a few thousand parallel processes.

Therefore, we corrected the errors in the algorithmic tangent by implementing a new kernel for the linear momentum balance equation. Currently, this new kernel is on a side branch of MOOSE (https://github.com/Argonne-National-Laboratory/deer), but we hope to merge it into baseline MOOSE in the future. 
Initial study of notch sensitivity of Grade 91 using mechanisms motivated crystal plasticity finite element method

September 2019

\section{Implementation and improvements of the grain boundary cavitation model}

\subsection{Small-strain cohesive zone framework in MOOSE}

The tensor_mechanics module available in MOOSE is formulated to use displacements as variables and forces as work conjugates. The cohesive zone model (CZM) that has been implemented in MOOSE utilizes a small-deformation discontinuous Galerkin approach, requires the use of a traction-separation law of the form $\mathbf{T}=f(\Delta \mathbf{u})$, and does not require the explicit addition of cohesive elements [37].

The implemented CZM utilizes a non-monolithic mesh to allow for a displacement jump $\Delta \mathbf{u}$ at the interface and adds additional surface quadrature points on one side of the interface to compute the traction $\mathbf{T}$ and store state variables. The displacement jump between two initially coincident points is defined as $\Delta \mathbf{u}:=\mathbf{u}^{-}-\mathbf{u}^{+}$, where superscripts + and - identify the positive and negative interface surfaces.

In general, a body subject to external forces must satisfy force equilibrium and angular momentum balance. For small-deformations the interface area $A$ and the interface normal $\mathbf{n}$ are assumed to be constant throughout the deformation and equilibrium is imposed on the initial mesh configuration. From a mechanistic perspective the interface surfaces are coincidental therefore automatically satisfying the angular momentum balance and only required to enforce equilibrium equation 4.1 .

$$
\mathbf{P}^{+}=\mathbf{P}^{-}
$$

The force acting over an area $A$ on an interface can be expressed as:

$$
\mathbf{P}=\int_{A} \mathbf{T} d A
$$

and therefore the strong form of the equilibrium equation can be rewritten as

$$
\int_{A^{+}} \mathbf{T}^{+} d A^{+}=\int_{A^{-}} \mathbf{T}^{-} d A^{-}
$$

For small-deformations the areas on both sides are equal, i.e. $d A^{+}=d A^{-}$, therefore equation 4.3 is satisfied only if the traction on both side are equal, e.g. $\mathbf{T}^{+}=\mathbf{T}^{-}$. In finite element methods, equilibrium is weakly enforced and the weak form of equation 4.3 can be obtained by multiplying the argument of the integrals by the appropriate test functions $\Psi$ :

$$
\int_{A} \mathbf{T} \Psi^{+} d A=\int_{A} \mathbf{T} \Psi^{-} d A
$$

The left hand side of equation 4.4 is imposed as an additional residual contribution to the nodes belonging to the positive interface while the right hand side is imposed on the nodes belonging to the negative interface. 
Initial study of notch sensitivity of Grade 91 using mechanisms motivated crystal plasticity finite element method

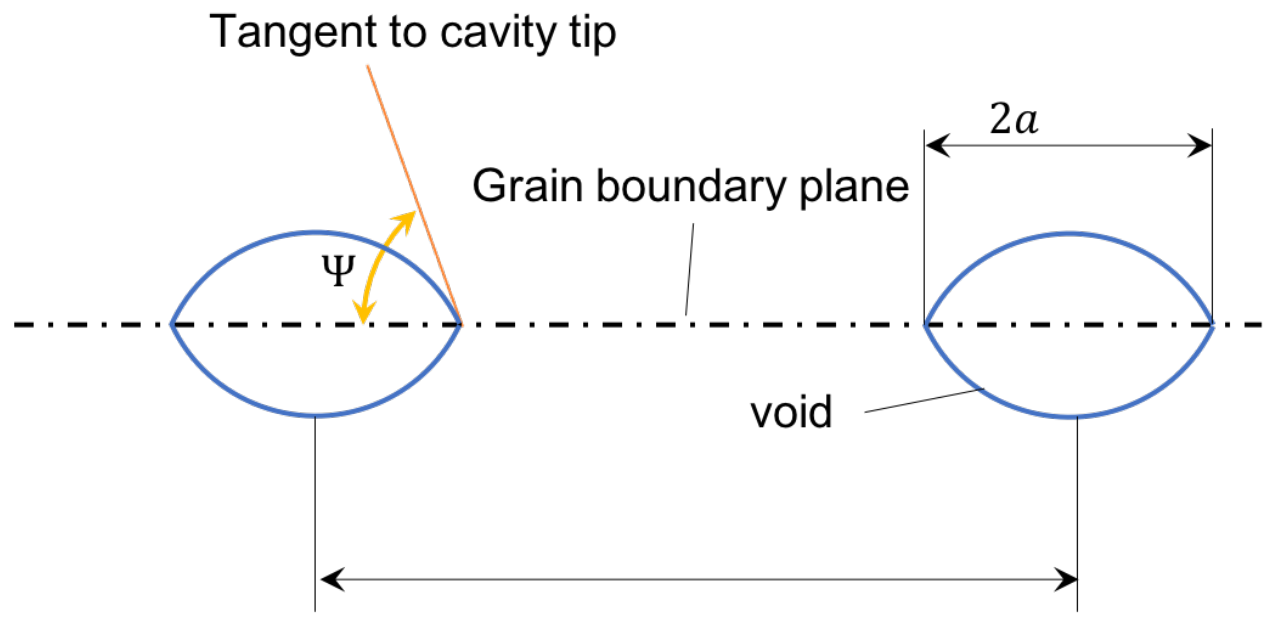

$2 b$

Figure 4.1: Schematic representing the geometrical quantities used in the grain boundary cavitation model and their physical meaning. $a$ : void half radius, $b$ : void-to-void half distance, and $\Psi$ : cavity half tip angle.

\subsection{Implementation and improvement of the grain boundary cavitation model}

Since last year's report [37], we focused on implementing and improving the grain boundary cavitation model described in Nassif et al. [38] in MOOSE. This model provides a traction separation law usable in any CZM approach and describes the void growth and nucleation via rate equations. The model assumes that surface diffusion on the void surface is rapid enough so that the voids maintain spherical-cap shapes with a half tip angle $\Psi$ (see Figure 4.1). The cavity growth and nucleation process are described in terms of two geometrical variables $a$ and $b$, which represent the average cavity half radius and the average half spacing, respectively. The ratio between $a$ and $b$ is closely related to the GB porosity and is a direct measure of the interface damage. When $a=b$ cavities coalesce and the interface is broken. The interface opening rate $\dot{\delta}_{N}$ is therefore constitutively linked to the cavity volumetric growth rate via $a$ and $b$.

The model in Nassif et al. [38] utilizes the following rate equations to describe the interface opening, cavity growth and cavity nucleation:

$$
\begin{aligned}
\dot{\delta}_{N} & =\frac{\dot{V}}{\pi b^{2}} \\
\dot{a} & =\frac{\dot{V}}{4 \pi h(\Psi) a^{2}} \quad \text { with } \quad a \in\left[a_{0}, b\right] \\
\dot{b} & =-\pi b^{3} F_{N}\left(\frac{\left\langle T_{N}\right\rangle}{\Sigma_{0}}\right)^{\gamma} \dot{\varepsilon}_{e q}^{C} \quad \text { active if } \quad\left(\frac{\left\langle T_{N}\right\rangle}{\Sigma_{0}}\right)^{\beta} \varepsilon_{e q}^{C} \geq \frac{N_{I}}{F_{N}} \quad \text { with } \quad b \geq b_{\text {sat }}
\end{aligned}
$$

where $\dot{V}$ is the cavity volumetric growth rate, $h$ (Eq. 4.17) is a function of the cavity half tip angle, $F_{N}$ is a constant affecting the nucleation rate, $\Sigma_{0}$ is a stress normalization factor, $N_{I}$ is 
Initial study of notch sensitivity of Grade 91 using mechanisms motivated crystal plasticity finite element method

September 2019

the initial cavity number area density, $a_{0}$ is the initial cavity radius and $b_{\text {sat }}$ is the minimum allowed half cavity spacing. The value of $b_{s a t}$ is computed in terms of the parameter $N_{\max }$, which represents the maximum allowed cavity number density. Note that the cavity half spacing $b$ is directly related to the cavity density $N$ by equation 4.8. Usually, the cavity nucleation rate equation is presented in terms $N$, however in this document we will use the cavity half spacing $b$ instead of $N$ to better expose the relationship between the varius equations and to limit the number of variables.

$$
N=\frac{1}{\pi b^{2}}
$$

Experimental results show that different mechanisms are responsible for cavity nucleation. In [12] it was reported that cavity nucleation is a continuous process primarily driven by the creep strain rate of grains adjacent to the interface. Lim [32] studied the contribution of grain boundary sliding to cavity nucleation and found that pure sliding does not contribute significantly to cavity nucleation and suggested that before a cavity can start growing a certain creep damage should be accumulated in adjacent grains. Wu and Sandstrom [53] studied the parameters governing the cavity nucleation process both experimentally and through modeling. They found that in most cases the cavity density is proportional to the creep strain. The grain boundary cavity growth model previously implemented in WARP3D and now in MOOSE was conceived by Needleman and Rice [40] and Sham and Needleman [46] and extended to a wide range of traixilities by (Van der Giessen et al. [51]). Van der Giessen et al. [51] utilized axisymetric finite element simulations to compute the growth rate of a single cavity under different loading conditions and generated a simplified model to represent the FE results. It should be emphasized that, as in WARP3D, the finite element implementation will not track individual cavities but will describe their mean evolution at each interface quadrature point. Therefore quantities such as $a$ and $b$ should be interpreted as the average behavior of a collection of cavities living on the area of influence of an interface material point.

The cavity growth model describes the the evolution of the cavity half radius $a$ in terms of a volumetric growth rate that is expressed in terms of cavity half spacing $b$, an average cavity size $a$, the normal traction $T_{N}$, and a few non local variable expressing the state of neighboring grains.

The cavity volumetric growth rate $\dot{V}$ has two terms, one to describe the initial cavity growth process $\dot{V}_{L}$ and one to describe void-void in-plane interaction, $\dot{V}_{H}$. It then assumes the cavity nucleation rate to be the largest in magnitude between $\dot{V}_{L}$ and $\dot{V}_{H}$ (Eq. 4.9). Both volumetric cavity growth rate formulations include a diffusion related term (Eqs. 4.10 and 4.13), which is identified by the superscript $D$, and a triaxial stress related term (Eqs. 4.12 and 4.15), which is identified by the superscript triax. The stress triaxility is defined as the ratio between a mean stress and an equivalent stress. For the grain boundary cavitation model we will use the hydrostatic stress, $s_{H}$, and the Von Mises equivalent stress $s_{V M}$ to compute the stress triaxility ratio. For interface calculations an average value of hydrostatic stress, von Mises stress and creep rate will be used. Such average nonlocal quantities are defined as: $s_{H}=0.5\left(s_{H}^{+}+s_{H}^{-}\right), s_{V M}=0.5\left(s_{V M}^{+}+s_{V M}^{-}\right)$and $\dot{\varepsilon}_{e q}^{C}=\sqrt{\frac{2}{3} \dot{\varepsilon}_{i, j}^{C} \dot{\varepsilon}_{i, j}^{C}}$. In the previous equations $s_{H}=\operatorname{tr}(\sigma) / 3, s_{V M}=\sqrt{\frac{3}{2} \sigma_{i, j} \sigma_{i, j}}$ and $\dot{\varepsilon}^{C}=\left(0.5\left(\dot{\varepsilon}^{C,+}+\dot{\varepsilon}^{C,-}\right)\right)$. 


$$
\dot{V}=\left\{\begin{array}{lll}
\dot{V}_{L}=\dot{V}_{L}^{D}+\dot{V}_{L}^{\text {triax }} & \text { if } & \left|\dot{V}_{L}\right| \geq\left|\dot{V}_{H}\right| \\
\dot{V}_{H}=\dot{V}_{H}^{D}+\dot{V}_{H}^{\text {triax }} & \text { if } & \left|\dot{V}_{H}\right|>\left|\dot{V}_{L}\right|
\end{array}\right.
$$

The triaxial contributions to void growth are further subdivided into two regimes, according to the triaxiality stress ratio.

$$
\begin{gathered}
\dot{V}_{L}^{D}=8 \pi D \frac{T_{N}}{q\left(f_{L}\right)} \\
\text { with } f_{L}=\max \left(\frac{a^{2}}{(a+1.5 L)^{2}}, \frac{a^{2}}{b^{2}}\right), \quad L=\left(\frac{D \sigma_{V M}}{\dot{\varepsilon}_{e q}^{C}}\right)^{\frac{1}{3}} \\
\dot{V}_{L}^{\text {triax }}= \begin{cases}2 \dot{\varepsilon}_{e q}^{C} a^{3} \pi h(\Psi) m\left\{\alpha_{n}\left|\frac{\sigma_{H}}{\sigma_{V M}}\right|+\beta_{n}(m)\right\}^{n} & \text { if }\left|\frac{\sigma_{H}}{\sigma_{V M}}\right| \geq 1 \\
2 \dot{\varepsilon}_{e q}^{C} a^{3} \pi h(\Psi)\left\{\alpha_{n}+\beta_{n}(m)\right\}^{n} \frac{\sigma_{H}}{\sigma_{V M}} & \text { if }\left|\frac{\sigma_{H}}{\sigma_{V M}}\right|<1\end{cases}
\end{gathered}
$$

where $n$ is the cavity growth exponent, $q$ is a function accelerating the degradation of the interface as function of the porosity, $h$, is a geometrical quantity, $m$ is the sign of the hydrostatic stress, $\beta$ and $\alpha_{n}$ are constants depending on $n$ and $m$. All the above quantities are defined in Equations 4.16 through 4.21.

$$
\begin{aligned}
& \dot{V}_{H}^{D}=8 \pi D \frac{T_{N}}{q\left(f_{H}\right)} \\
& \text { with } f_{H}=\left(\frac{a^{2}}{b^{2}}\right) \\
& \dot{V}_{h}^{\text {triax }}= \begin{cases}2 \dot{\varepsilon}_{e q}^{C} a^{3} \pi h(\Psi) m\left\{\frac{\left[\alpha_{n}\left|\frac{\sigma_{H}}{\sigma_{V M}}\right|+\frac{m}{n}\right]}{\left(1-0.87 \frac{a}{b}\right)^{\frac{3}{n}}}\right\}^{n} & \text { if }\left|\frac{\sigma_{H}}{\sigma_{V M}}\right| \geq 1 \\
2 \dot{\varepsilon}_{e q}^{C} a^{3} \pi h(\Psi)\left\{\frac{\left[\alpha_{n}+\frac{m}{n}\right]}{\left(1-0.87 \frac{a}{b}\right)^{\frac{3}{n}}}\right\}^{n} \frac{\sigma_{H}}{\sigma_{V M}} & \text { if }\left|\frac{\sigma_{H}}{\sigma_{V M}}\right|<1\end{cases}
\end{aligned}
$$




$$
\begin{aligned}
& q(f)=2 \log \left(\frac{1}{f}\right)-(1-f)(3-f) \\
& h(\Psi)=\left(\frac{1}{1-\cos (\Psi)}-\frac{\cos (\Psi)}{2}\right) \frac{1}{\sin (\Psi)} \\
& m=\operatorname{sign}\left(\sigma_{H}\right) \\
& \beta(m)=\frac{(n-1)[n+g(m)]}{n^{2}} \\
& g(m)=\left\{\begin{array}{lll}
\log (3)-\frac{2}{3} & \text { if } & m=1 \\
\frac{2 \pi}{9 \sqrt{3}} & \text { if } & m=-1 \\
0 & \text { if } & m=0
\end{array}\right. \\
& \alpha_{n}=\frac{3}{2 n}
\end{aligned}
$$

A traction separation law also requires the in-plane traction to be defined in 3D. In general the two in-plane directions are chosen arbitrarily. The only requirement is the two directions be orthogonal. This choice implies isotropic in-plane behavior of the interface. For brevity we will name the transverse directions as 1 and 2 and by utilizing the notation $1 / 2$ it is implied that such relationship applies for both transverse directions. During creep it assumed that grain boundary sliding occurs. The model implements the following sliding equations:

$$
T_{S_{1 / 2}}=\eta_{S} f_{S}\left(\frac{a}{b}\right) \dot{\delta}_{1 / 2} \quad \text { with } \quad f_{S}\left(\frac{a}{b}\right)=\left\{\begin{array}{l}
1 \quad \text { if } \quad \frac{a}{b} \leq 0.5 \\
2\left(-\frac{a}{b}+1\right) \quad \text { if } \quad \frac{a}{b}>0.5
\end{array}\right.
$$

In summary, the model has the following characteristics:

- The model assumes continuous cavity nucleation and growth.

- The traction separation law is purely viscous.

- Nucleation (Eq. 4.7) can occur only under tension and if the nucleation criterion is met. If both criteria are met the cavity nucleation rate is proportional to the equivalent creep rate $\dot{\varepsilon}_{e q}^{C}$ and to $T_{N}^{\gamma}$.

- Newly nucleated cavities are assumed to instantly reach the $a$ value of already present cavities. 
Initial study of notch sensitivity of Grade 91 using mechanisms motivated crystal plasticity finite element method

September 2019

- The shape of the cavity is fixed by the angle $\Psi$, thus assuming that the time required for a cavity to reach its quasi-equilibrium shape is much smaller than the time required to grow or nucleate.

- The cavity half spacing $b$ can only decrease ( $N$ can only increase).

- The cavity volume can increase or decrease depending on the sign of $T_{N}$ and $\sigma_{H}$, however the lower bound of the cavity radius is limited to $a=a_{0}$ by the fact that cavities nucleate from precipitates segregated at grain boundaries.

- Cavity growth and nucleation are not affected by sliding.

\subsubsection{Model improvements}

\subsubsection{Visco-elastic grain boundary response}

One of the goals of this project is to eventually simulate the creep-fatigue response of Grade 91. Utilizing a purely viscous traction-separation law might cause numerical issues because equations are stiff when load changes happen. Examples of this behavior are: the initial load ramp for a creep simulation, load reversal experienced during fatigue loading or interface failure. Another consideration is that materials, even under high temperature conditions, still exhibit elastic behavior when load changes happens in a short timeframe. We already experienced numerical issues in WARP3D simulations when ramping the load from the initial condition to the imposed creep stress, and the only solution was to utilize a very small time step when increasing the load. Therefore we decided to improve the model by utilizing a Maxwell model for the traction separation law. In a purely viscous model traction are only related to the velocity as:

$$
T_{N}=\eta \dot{\delta}_{N}
$$

The Maxwell model can be seen as a spring in series with a dashpot. The dashpot is responsible for stress relaxation and the spring is responsible for responding to instantaneous load changes. The rate equation for a Maxwell model representing an interface can be written as:

$$
\dot{T}_{N}=\left(\dot{\delta}_{N}+\frac{T_{N}}{\eta}\right) C
$$

where $C$ is the interface stiffness. By comparing Equations 4.23 and 4.5 one obtains

$$
\frac{T_{N}}{\eta}=\frac{\dot{V}}{\pi b^{2}}
$$

and substituting in equation 4.24 the traction rate equation becomes:

$$
\dot{T}_{N}=\left(\dot{\delta}_{N}+\frac{\dot{V}\left(T_{N}\right)}{\pi b^{2}}\right) C \quad \text { with } \quad C=\frac{E\left(1-\frac{a}{b}\right)}{W}
$$


Initial study of notch sensitivity of Grade 91 using mechanisms motivated crystal plasticity finite element method

September 2019
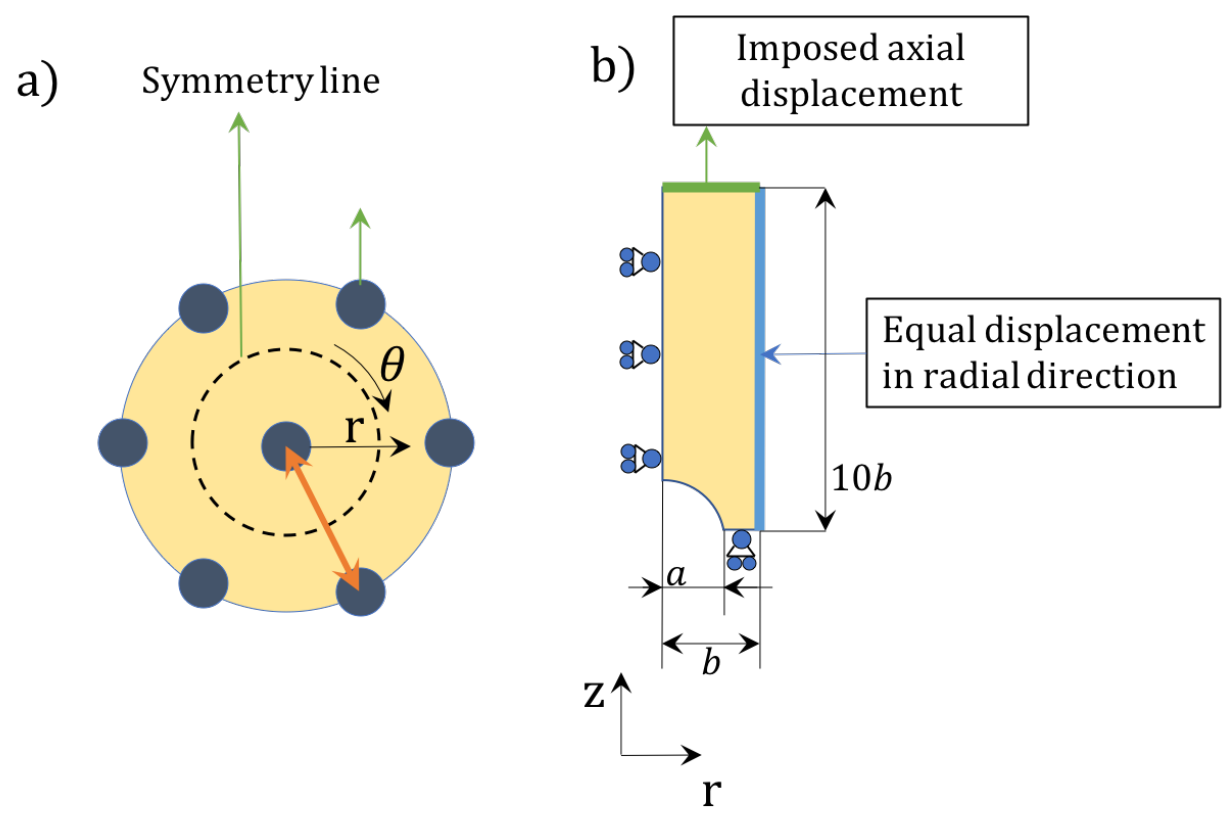

Figure 4.2: a): schematic representing the location of cavities on the grain boundary plane assuming axial symmetry; b) Schematic depicting the axisymmetric model used to identify the interface thickness, including the imposed boundary conditions.

where $E$ is the interface Young modulus, and $W$ is the interface thickness. The $a / b$ term in the stiffness equation includes the effect of damage by reducing the stiffness as damage progresses. Note that in case of isotropic elasticity the young modulus $E$ is equal to the value in one of the grains. In what follows Eq. 4.26 will replace Eq. 4.5

In a finite element framework the element stiffness is directly linked to the element size. For a cohesive element the geometrical thickness in the opening direction is usually zero at the beginning. However, in most cases one is required to assign a fictitious element thickness that should be representative of the region of material in which the fracture process takes place. In this case the interface thickness can be computed using axisymmetric, elastic finite element simulations by identifying the distance from the grain boundary plane after which the stress field becomes constant (see Fig. 4.2 a). To obtain a conservative estimate of the interface thickness $W$, we performed a set of simulations for different $a / b$ ratios ranging from 0.1 to 0.98 . For all the simulations the same displacement $u_{z}=0.1 b$ has been applied on the top surface while an equal value radial displacement boundary condition was imposed on the right surface. The equal value boundary condition was selected to not over or under constrain the system. Figure 4.2.b is a schematic depicting the applied boundary conditions.

The criterion selected is the distance from the grain boundary plane for which the gradient of the stress becomes small, specifically $\nabla \sigma_{V M}<0.1$. Results show that the stress gradient is below the selected threshold after a distance $L=3 b$ (see Fig. 4.3). The interface thickness was therefore selected as $W=2 L=6 b$ to include both sides. Furthermore, the value of $b$ continuously decreases up to an imposed minimum value $b=b_{\text {sat }}=\frac{1}{\sqrt{\pi N_{\max }}}$, where $N_{\text {max }}$ is a model parameter representing the maximum allowed cavity density. To be conservative, we selected the largest obtainable interface stiffness, i.e. $W=6 b_{s a t}$. 


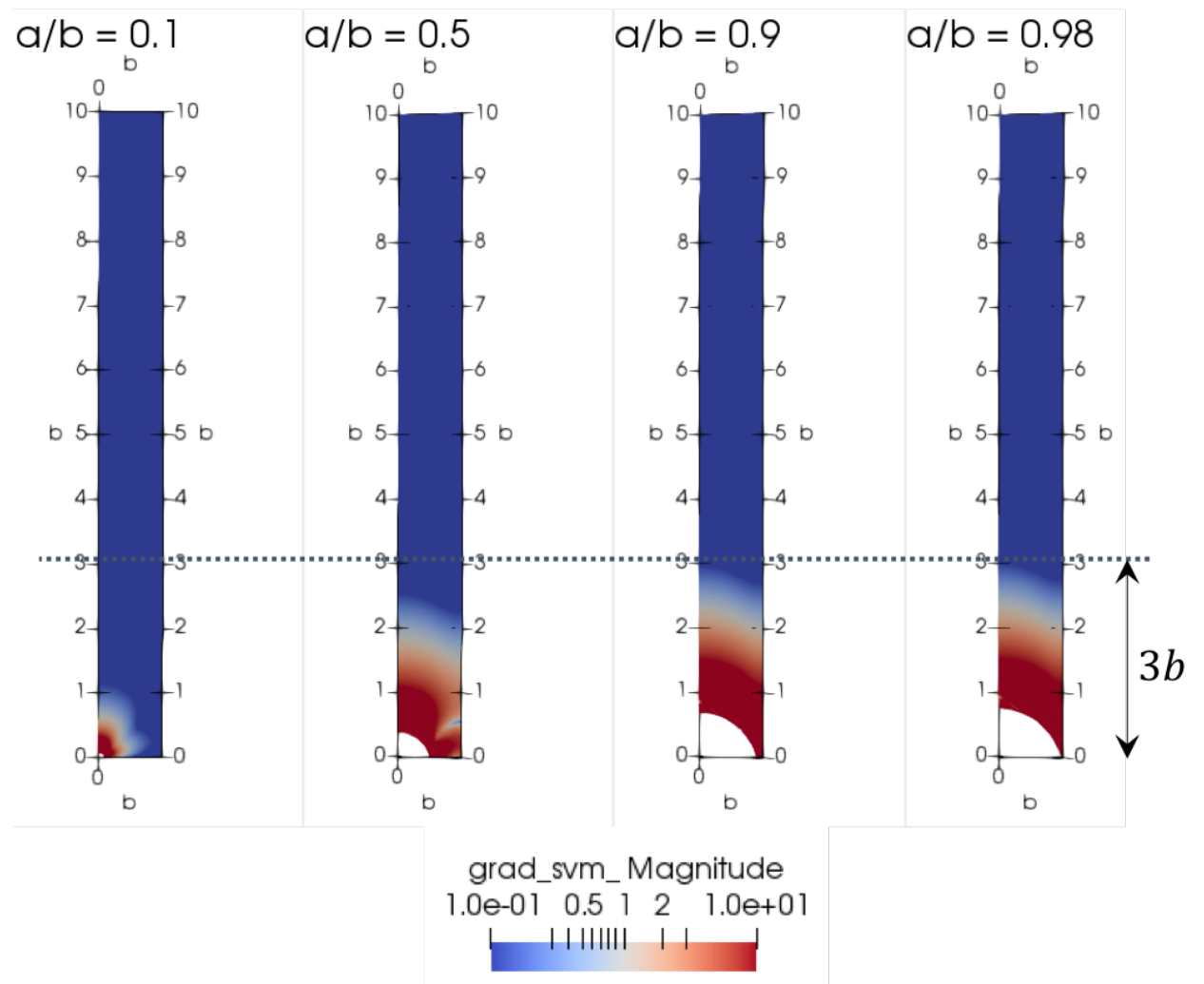

Figure 4.3: Contour plot depicting the von Mises stress gradient field for different $a / b$ ratios. For a cavity with $a / b=0.98$ the stress becomes constant after a distance $3 b$ from the grain boundary plane. 
Initial study of notch sensitivity of Grade 91 using mechanisms motivated crystal plasticity finite element method

September 2019

It should be noted that linking the interface thickness to the model parameter $N_{\max }$ introduces a length scale in the problem and this approach is justified if the resulting interface thickness $W$ is smaller than the characteristic bulk element length. In general the interface element stiffness should be larger than the that of bulk elements to not allow separation before damage is initiated. This assumption will be verified later in this chapter.

The Maxwell model has also been used for the shear traction:

$$
\dot{T}_{S, 1 / 2}=\left(\dot{\delta}_{S, 1 / 2}+\frac{T_{S, 1 / 2}}{\eta_{S} f_{S}}\right) \frac{6 b_{s a t}}{G\left(1-\frac{a}{b}\right)} \quad \text { with } \quad f_{S}\left(\frac{a}{b}\right)=\left\{\begin{array}{l}
1 \quad \text { if } \quad \frac{a}{b}=<0.5 \\
2\left(-\frac{a}{b}+1\right) \quad \text { if } \quad \frac{a}{b}=\geq 0.5
\end{array}\right.
$$

where $G$ is the interface shear modulus, which is, for isotropic elasticity, $G=\frac{E}{2(1+\nu)}$, with $\nu$ being the Poisson's ratio of the neighboring grain.

Another change made to the model was to modify the activation condition of the nucleation rate equation (see Eq. 4.7) from being always required to be a one time check:

$$
\dot{b}=-\pi b^{3} F_{N}\left(\frac{\left\langle T_{N}\right\rangle}{\Sigma_{0}}\right)^{\gamma} \dot{\varepsilon}_{e q}^{C} \quad \text { active if } \quad\left(\frac{\left\langle T_{N}\right\rangle}{\Sigma_{0}}\right)^{\beta} \int_{0}^{T}\left|\dot{\varepsilon}_{e q}^{C}\right| d t \geq \frac{N_{I}}{F_{N}} \quad \text { once }
$$

Physically this interpretation suggests that when the threshold value is achieved cavities can continuously nucleate under any positive normal traction. With this modification we argue that after enough creep strain has been accumulated the cavity nucleation process is continuous. This is still in agreement with nucleation threshold value proposed by Lim [32], however it is in contrast with the previous interpretation where the nucleation process could be intermittent if the normal traction drops significantly. This approach makes the problem numerically smoother. In practice, the two different activation strategies are almost equivalent for creep loading conditions because the opening traction exhibits a smooth behavior with limited variation in time Messner et al. [34], and therefore once the threshold is met nucleation will continue in both cases. However, different responses could be possible under creep fatigue loading conditions where the applied load can vary drastically in a short time.

\subsubsection{Modeling interface failure}

Interface failure ideally happens when the damage $D=a / b=1$. However, reaching a value of $D=1$ is impossible, especially for load controlled simulations, as it results in numerical instability. A practical approach to solve this issue is to choose a critical damage value $D_{c r}<1$, after which damage stops evolving leaving an arbitrarily small residual stiffness to improve the numerical stability of the simulations. However, if $D_{c r}$ is not close enough to 1.0 (e.g. $1-D_{c r}<10^{-3}$ ) the residual interface stiffness would still be high enough to influence the simulation results. On the other hand reaching a value of $D=0.999$ requires a large computational effort and might trigger global time steps reduction too often, thus making CPFEM creep simulations almost unfeasible from a computational cost perspective. We therefore adopted a strategy to avoid excessive time step reductions while still neutralizing the interface stiffness. 
Initial study of notch sensitivity of Grade 91 using mechanisms motivated crystal plasticity finite element method

September 2019

Our strategy relies on four checks to determine if an interface material point is ready to be marked as failed:

- The extrapolated time required to achieve an interface damage $D=1$ is smaller than a fixed critical time $t_{c r}$. The time to complete failure is computed by extrapolating the current damage rate as: $t_{\text {residual life }}=\frac{1-D}{\dot{D}}$, where both $D$ and $\dot{D}$ are evaluated after a converged solution has been achieved. The interface material point is marked as failed if $t_{\text {residual life }} \leq t_{c r}$. This check avoids triggering global timestep reduction because of load redistribution issues or an excessive number of adaptive material substeps.

- The damage of an interface material point exceeds a critical value $D_{c r}$. A typical value is $D_{c r}=0.95$.

- The opening traction $T_{N}$ exceeds a critical value $T_{N, c r}$. This check is necessary to mark as failed elements during the last stages of the simulation to avoid excessive bulk elements distortion. A typical value is $T_{N, c r}=1400 \mathrm{MPa}$.

- A check for an unreasonable situation deriving from the fact that equations do not allow for cavities to accumulate damage by changing shape and becoming oblate. This check is is triggered if and only if the interface damage rate $\dot{D}>0$ and the normal traction $T_{N}<0$. Failure occurs when this condition is met and either: (i) the extrapolated residual life is smaller than $t_{c r} O R$ (ii) during a time increment of size $t_{c r}$ the extrapolated traction would result in a negative value with a magnitude larger than $T_{N, c r}$. Condition (ii) is computed as $\dot{T}_{N} t_{c r}+T_{N}<-\left|T_{N, c r}\right|$, where both the normal traction and its rate are evaluated after convergence is achieved. The reason for including this check will be further explained later in this chapter.

If any of the above occurs, the material point is marked as failed. The time at which failure occurs $t_{\text {fail }}$, the displacement jump vector at failure $\Delta u_{\text {fail }}$, and all the traction component at at failure $T_{\text {fail }}$ are recorded and set as constants for the rest of the simulation. When a material point has been marked as failed an exponential decay relationship is used to reduce the stiffness and bring the tractions to zero:

$$
\begin{aligned}
T_{i} & =\left[\left(\Delta u_{i}-\Delta u_{i, f a i l}\right) C_{i}+T_{i, f a i l} D F\right] \quad \text { where: } \\
C_{i} & =\max \left(\frac{T_{i, \text { fail }}}{\Delta u_{i, f a i l}} D F, C_{\text {min }}\right) \\
D F & =\exp \left(-\frac{\left(t-t_{\text {fail }}\right)}{0.5 t_{\text {residual life }}}\right)
\end{aligned}
$$

where $i$ is an index ranging from 1 to 3 identifying either the normal or one of the sliding directions, $C_{i}$ is the current stiffness of the interface, $C_{m i n}$ is the minimum allowed stiffness (typical value $C=1 M P a m$ ), and $D F$ is an exponential decay factor applied to reduce both the interface stiffness and traction at failure in a time frame $\Delta t=t_{\text {residual life }}$.

This approach allows the interface to retain a small $C=C_{\min }$ and prevents disconnected domains, which are numerically unstable in implicit finite element frameworks. It skips calculations for interface material residual lives smaller than $t_{c r}$ (typical value $50 \mathrm{~h}$ ). In fact 
Initial study of notch sensitivity of Grade 91 using mechanisms motivated crystal plasticity finite element method

September 2019

most of the calculation cost is related to the last portion of an element life. In general such small residual lives can be neglected from an engineering perspective if a reasonable $t_{c r}$ is selected. For instance even if the entire creep life is $1000 \mathrm{~h}$ selecting a $t_{c r}=50 \mathrm{~h}$ would still only account for $5 \%$ of the entire creep life.

\subsubsection{Numerical implementation}

As in the WARP3D implementation, the bulk quantities required by the interface model, such as $\sigma_{H}, \sigma_{V M}$, and $\dot{\varepsilon}_{e q}^{C}$ are staggered. This means that at time step $n+1$ the cavitation models utilizes values computed at time step $n$. This also implies that the triaxial branch for the cavity growth rate is decided at the beginning of the timestep.

The numerical implementation of the cavitation model ensures that simulations can proceed after the onset of tertiary creep and generate experimentally observed failure strains. As noted above, the cavity volumetric growth rate equations have different branches which are selected depending on the current material state. One of the main concerns in [34] was that simulation were failing to achieve high strain rates. We believe this occurred because of the discontinuous nature of the model due to the presence of hard max functions. These discontinuous functions severely affect the convergence of the local implicit time integration. Therefore, we substituted all the max functions with the so called smoothmax to obtain a continuous system of equations. The smoothmax function implemented in the material model is based on [10] and modified to automatically adjust its sharpness depending on values of $x$ and $y$ :

$$
\operatorname{smoothmax}(x, y)=\log \left(\exp \left(\frac{f x}{|x|+|y|}\right)+\exp \left(\frac{f y}{|x|+|y|}\right)\right) \frac{|x|+|y|}{f}
$$

where $f$ is a scaling factor which governs the sharpness of the smoothmax. We found that a value of $f=50$ provides good results independently from the values of $x$ and $y$. In the implementation the smoothmax function was applied to equations 4.9, 4.11, and 4.28 and simulations use a value of $f=50$ (see Eq. 4.33). The rate equations used in the final nonlinear system are shown in Eq. 4.33.

All the grain boundary cavitation model equations have been presented in terms of rates. Some of the variables have physical constraints that are naturally specified in terms of variable values and not rates. The physical constraints in the systems are:

- the new value of $a$ for time step $n+1$ must be larger or equal to $a_{0}$ and smaller than $b^{n+1}: a_{0} \leq a^{n+1} \leq b^{n+1}$.

- the new value of $b$ for time step $n+1$ must be smaller or equal to $b^{n}$ and larger than $b_{\text {sat }}: b^{n} \geq b^{n+1} \geq b^{\text {sat }}$.

The lower bounds for the variables in the cavity growth equation are imposed by means of the smoothmax function. The minimum value $a$ is imposed by implementing smoothmax $\left(a_{0}, \theta^{M}(\dot{a})\right)$, where $\theta^{M}(\cdot)$ implies utilizing the $\theta$ - method (cfr. Eq. 4.35 ) to integrate the rate equation (see also Eqs. 4.34). The smoothmax function is also used to replace the Macaulay brackets in Eq. 4.28 with $\left\langle T_{N}\right\rangle=$ smoothmax $\left(1 e-3, T_{N}\right)$ (see Eqs. 4.33), thus not allowing negative 
Initial study of notch sensitivity of Grade 91 using mechanisms motivated crystal plasticity finite element method

September 2019

values of $T_{N}$ and therefore implicitly imposing that $b$ can only evolve for positive values of the $T_{N}$.

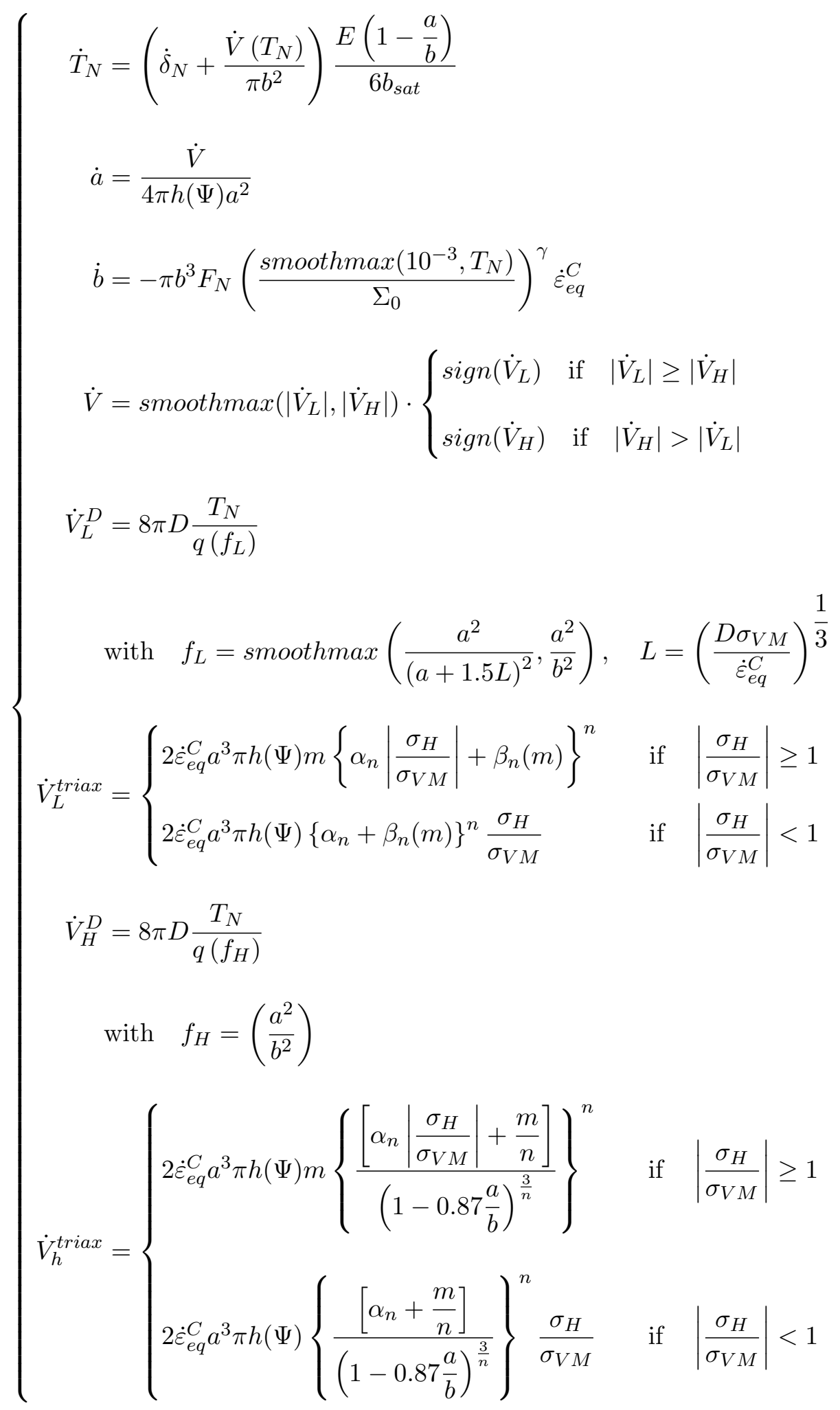

Including the two upper constraints in the material nonlinear solver results in adding two 
Initial study of notch sensitivity of Grade 91 using mechanisms motivated crystal plasticity finite element method

September 2019

additional equations, thus increasing the numerical burden required to solve each interface material point. The requirement $b \leq b_{\text {sat }}$ is arbitrary, therefore we decide to loosely enforce it by inhibiting nucleation if $b^{n} \geq b_{\text {sat }}$. The requirement on $a$ and $b$ are not enforced during the solution of the nonlinear system but only after convergence has been achieved.

The implemented residual equations for the material nonlinear system are:

$$
\left\{\begin{aligned}
R_{T_{N}} & =\left(T_{N}^{n+1}-\theta^{M}\left(\dot{T}_{N}\right)\right) \frac{1}{10} \\
R_{a} & =\left(a^{n+1}-\operatorname{smoothmax}\left(a_{0}, \theta^{M}(\dot{a})\right)\right) \frac{1}{a^{n}} \\
R_{b} & =\left(b^{n+1}-\theta^{M}(\dot{b})\right) \frac{1}{b^{n}}
\end{aligned}\right.
$$

where $\theta^{M}$ means utilizing the $\theta$ method (see Eq. 4.35) to integrate the rate equations in 4.33. Furthermore, the fractions on the right side are scaling factors to normalize the residual values. The only residual equation that is not normalized according to the previous time step value is the one for the normal traction. For this equation a scaling factor of 10 has been selected to avoid unnecessary large residuals when load redistribution occurs.

Equation 4.34 defines a nonlinear system of equations that needs to be solved numerically. We utilized the SNES library available in PETSC [7], which is already available in MOOSE. This provides the flexibility to try different solvers before giving up on the solution of the nonlinear system and provides a finite difference algorithm. To solve the nonlinear system the interface material first tries a standard newton method for the entire timestep. If the standard solver fails, then an adaptive substep is used and a newton solver with line-search is adopted. The maximum allowed number of substeps can be specified by the user in the input file.

The $\theta$ method was used to integrate the rate equations:

$$
x^{n+1}=\left(\theta F^{n}+(1-\theta) F^{n+1}\right) \Delta t+x^{n}
$$

where $0 \leq \theta \leq 1, n$ is the time step index and $F$ is the function value. For $\theta=1$ one recovers the forward Euler (explicit) time integration scheme, $\theta=0$ one obtains the backward Euler (implicit) and for $\theta=0.5$ one obtains the Cranck-Nicolson method (second order, implicit) integration method. In our implementation, $\theta$ is a user defined parameter, thus allowing the user to decide which time integration scheme to use. In what follows we always used the backward Euler method (e.g $\theta=0)$.

The calculation for the shear traction is done independently from the normal traction and uses staggered values of $a$ and $b$ to make the shear tractions independent from the opening traction. This strategy was adopted for numerical efficiency and because shear tractions do not contribute to damage. This also allows us to use a closed form solution for the shear traction rate equation: 
Initial study of notch sensitivity of Grade 91 using mechanisms motivated crystal plasticity finite element method

September 2019

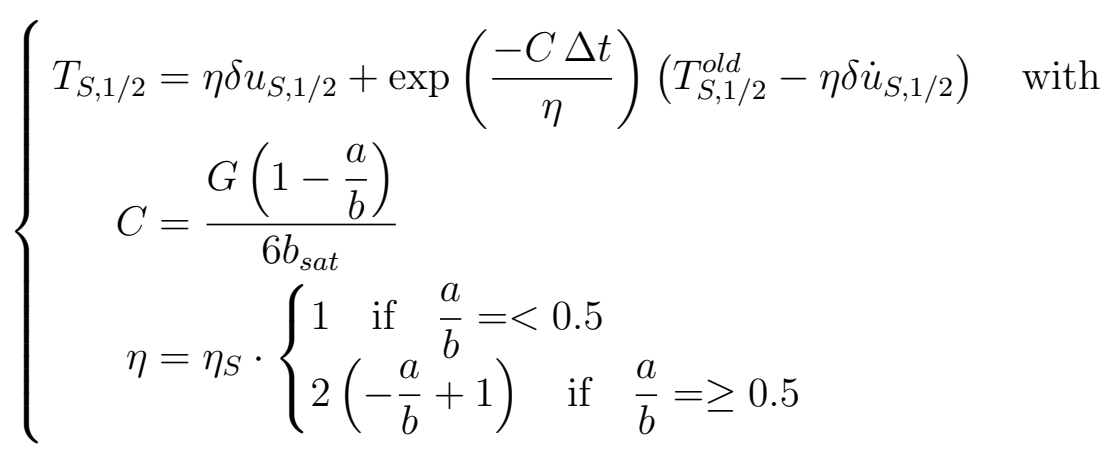

\subsubsection{Avoid bulk elements interpenetration}

The proposed traction separation rate relationship is suitable for a implementing a stiffness penalty methodology to prevent interpenetration. It should be noted that the module assumes an initial interface thickness $W=6 b_{\text {sat }}$, therefore interpenetration is assumed to happen only if the interface normal displacement $\delta_{N}<-W$. When this condition is met the interface stiffness $C$ is multiplied by a penalty factor $E_{\text {penalty }}=10$.

\subsubsection{Interface model parameters}

For all the results in this report the grain boundary cavitation model parameters are the same as those identified in Messner et al. [34] and are reported here for completeness.

\begin{tabular}{llll} 
symbol & description & value & units \\
\hline$\beta$ & traction nucleation exponent & 2 & unitless \\
\hline$n$ & creep rate exponent & 5 & unitless \\
\hline$a_{0}$ & initial cavities half radius & $5 \cdot 10^{-5}$ & $\mathrm{~mm}^{2}$ \\
\hline$b_{0}$ & initial cavities half spacing & 0.06 & $\mathrm{~mm}^{2}$ \\
\hline$D$ & grain boundary diffusion coefficient & $1 \cdot 10^{-15}$ & $\mathrm{~mm}^{3} / \mathrm{MPa} \cdot \mathrm{h}$ \\
$\Psi$ & cavity half tip angle & 75 & {$[\circ]$} \\
\hline$\Sigma_{0}$ & traction normalization paramter & 200 & $\mathrm{~mm}^{2}$ \\
\hline$\frac{F N}{N I}$ & normalized nucelation rate constant & $2 \cdot 10^{4}$ & $1 / \mathrm{mm}^{2}$ \\
\hline$\frac{N \text { max }}{N I}$ & normalized maximum cavity density & $1 \cdot 10^{3}$ & $1 / \mathrm{mm}^{2}$ \\
\hline$E_{\text {interface }}$ & interface Young modulkus & $150 \cdot 10^{3}$ & $1 / \mathrm{mm}^{2}$ \\
\hline$E_{\text {penalty }}$ & interpenetration penalty coefficinet & 10 & $\mathrm{unitless}$ \\
\hline$\eta_{S}$ & sliding visocisty & $1 \cdot 10^{6}$ & $\mathrm{unitless}$ \\
\hline$G_{\text {interface }}$ & interface in-plane Shear modulus & $52.63 \cdot 10^{3}$ & $1 / \mathrm{mm}^{2}$ \\
\hline theta & parameter for the time integration scheme & 0 & $\mathrm{unitless}$ \\
\hline
\end{tabular}

Table 4.1: Grain boundary cavitation material parameters.

Table 4.2 shows the typical damage parameters used for all the results presented in this work.

Table 4.3 shows the typical nonlinear solver parameters used to determine if the nonlinear system has converged. 
Initial study of notch sensitivity of Grade 91 using mechanisms motivated crystal plasticity finite element method

September 2019

\begin{tabular}{llll} 
symbol & description & value & units \\
\hline$D_{c r}$ & maximum damage before element fail & 0.9 & unitless \\
\hline$t_{c r}$ & minimu allowed traction residual life & 50 & $\mathrm{~h}$ \\
\hline$T_{N, c r}$ & maximum aloweed normal traction & 1400 & $\mathrm{MPa}$ \\
\hline
\end{tabular}

Table 4.2: Grain boundary cavitation damage parameters.

\begin{tabular}{llll} 
symbol & description & value & units \\
\hline abs_tol & absolute tolerance & $1 \cdot 10^{-6}$ & unitless \\
\hline rel_tol & relative tolerance & $1 \cdot 10^{-6}$ & unitless \\
\hline step_tol & step tolerance & $1 \cdot 10^{-6}$ & unitless \\
\hline
\end{tabular}

Table 4.3: Grain boundary cavitation SNES parameters. For more details the reader is referred to PESTC manual [6].

Table 4.4 shows the available options to change the behavior of the GB cavitation model.

\begin{tabular}{lcc} 
flag name & description & possible values \\
\hline \multirow{2}{*}{ vdot_type } & the type of volumetric growth rate used & 1 uses $\dot{V}_{L}$ \\
& & 2 uses $\dot{V}_{H}$ \\
& boolean enabling & $3 \dot{V}_{H}$ \\
triaxial_vdot_active & $\dot{V}_{L}^{\text {triax }}$ and or $\dot{V}_{H}^{\text {triax }}$ & true or false \\
& according to vdot type & \\
\hline max_substep_number & maximum number of substeps & any positive integer \\
\hline nucleation_active & boolean enabling the nucleation equation & true or false \\
\hline growth_active & boolean enabling the cavity nucleation equation & true or false \\
\hline use_SNES_FD & use PETSC finite difference for jacobian calculation & true or false \\
\hline
\end{tabular}

Table 4.4: Grain boundary model options.

\subsection{Model validation and testing}

The improved model was first tested to verify that the smoothmax function and all constraints are respected. Simple tests were performed on a mesh with two elements. Each element represents one grain. The two element simulations verify that the improved interface model is working as expected. Figure 4.4 shows the two element mesh utilized for testing. The characteristic size of bulk elements utilized in the two element simulation is comparable to the one that will be used for CPFEM simulations.

The first test applies uniaxial cyclic loading to the top $(z=Z)$ surface of the two 2 element simulation (see Table 4.5 for other boundary conditions). This simulation demonstrates that:

- cavity nucleation starts after accumulating a certain strain 
Initial study of notch sensitivity of Grade 91 using mechanisms motivated crystal plasticity finite element method

September 2019
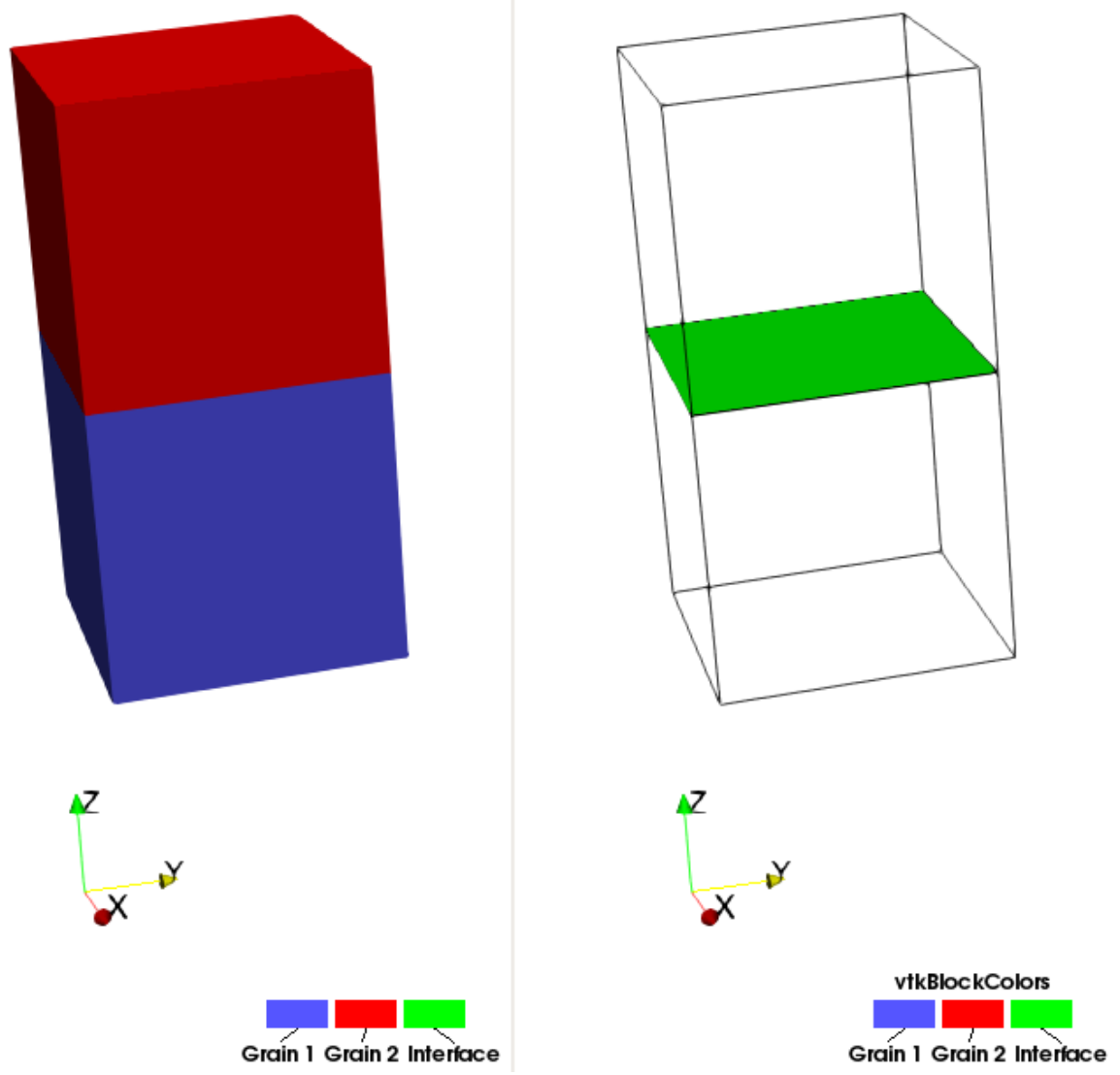

Figure 4.4: The two element mesh utilized to check the model behavior. On the left the mesh is colored by grain number, on the right the interface between the two grains is highlighted in green. 
Initial study of notch sensitivity of Grade 91 using mechanisms motivated crystal plasticity finite element method

September 2019

\begin{tabular}{llll} 
surface & disp $_{x}$ & disp $_{y}$ & disp $_{z}$ \\
\hline$x=0$ & 0 & free & free \\
$x=X$ & equalvalue & free & free \\
\hline$y=0$ & free & 0 & free \\
$y=Y$ & free & equalvalue & free \\
\hline$z=0$ & free & free & 0 \\
$z=Z$ & free & free & imposed
\end{tabular}

Table 4.5: Boundary conditions for the 2 element displacement controlled simulations.

- cavity nucleation is not active if the interface is under compression

- the cavity can increase or decrease in size

- the minimum cavity radius constraint is respected

- the interface is stiffer than bulk elements

Figure 4.5 shows the evolution of the cavity radius $a$ and half spacing $b$ versus time. The normal interface traction $T_{N}$ is also plotted for reference. The cavity radius increases while the normal traction is positive and decreases when the normal traction is negative. The fixed value of $a=5.5 \cdot 10^{-5}$ between 200 and 300 hours matches the imposed minimum cavity radius $a_{0}$. Around 300 hours the normal traction becomes positive again and the cavity radius increases. Looking at the cavity spacing plot, cavities start nucleating after approximately 25 hours. This is the effect of imposing a threshold on the cavity nucleation equation. After this point cavities nucleate quickly until the normal traction starts dropping (100 hours). When the normal traction becomes negative $b$ becomes stationary, and starts decreasing again when the traction becomes positive. The last plot depicts the interface strain versus and the grain strain in the loading direction versus time. Before damage starts accumulating the grains accommodate most of the deformation. This confirms that with an interface thickness of $W=6 b_{s a t}$ the pristine interface is stiffer than bulk elements. When damage starts accumulating the interface softens as expected.

Another three tests use the three different cavity growth volumetric rates $\left(\dot{V}_{L}, \dot{V}_{H}\right.$, and both together) and check whether simulations can proceed up to complete separation. The applied boundary conditions for the three simulations are identical and are listed in Table 4.6. Results are shown in Figures 4.6, 4.7, and 4.8, in which a) shows the final configuration after rupture (displacements are reduced by a factor of 1000), b) depicts the imposed traction and the interface displacement jump versus time and c) depicts the interface damage and the interface displacement jump versus time. For all simulations the critical damage $D_{c r}=0.9$ and $t_{c r}=50$ hours. All simulations are able to achieve complete separations. This validates the minimum residual stiffness approach presented in the previous section. The results also show that simulation can proceed up to very high damage fraction value without issues.

We then tested the improved interface model on a 15 grains microstructure subject to creep test conditions where a simple power law creep relationship is used to model grains deformation. The power law creep model evolves the fluidity utilizing a Voce law to simulate 
Initial study of notch sensitivity of Grade 91 using mechanisms motivated crystal plasticity finite element method

September 2019
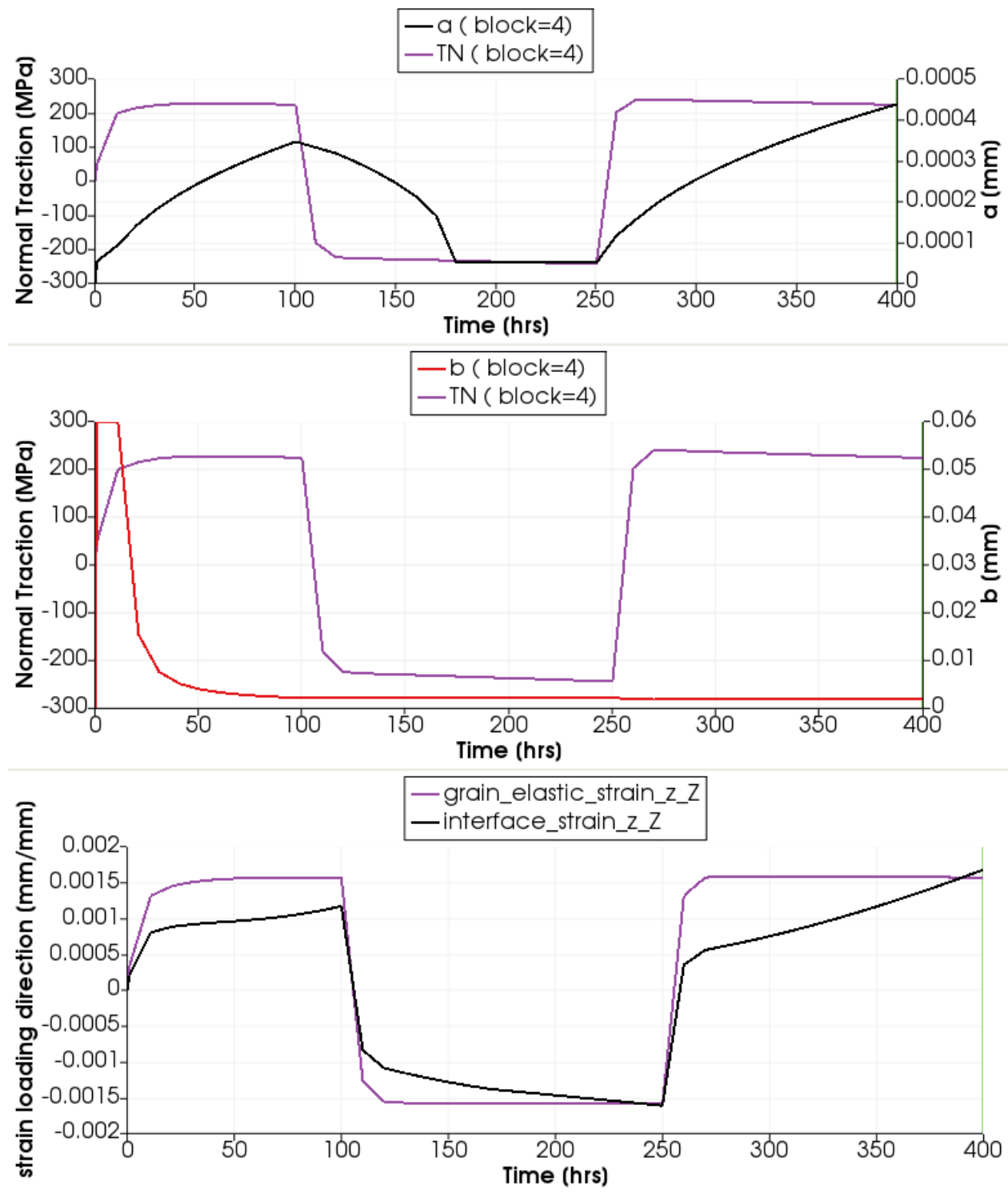

Figure 4.5: Results of the 2 element uniaxial cyclic test. a) cavity half radius $a$ and normal traction $T_{N}$ versus time, b) cavity half spacing $b$ and normal traction $T_{N}$ versus time, c) interface strain and grain elastic strain versus time. 
Initial study of notch sensitivity of Grade 91 using mechanisms motivated crystal plasticity finite element method

\section{Time: 2763 (hrs)}
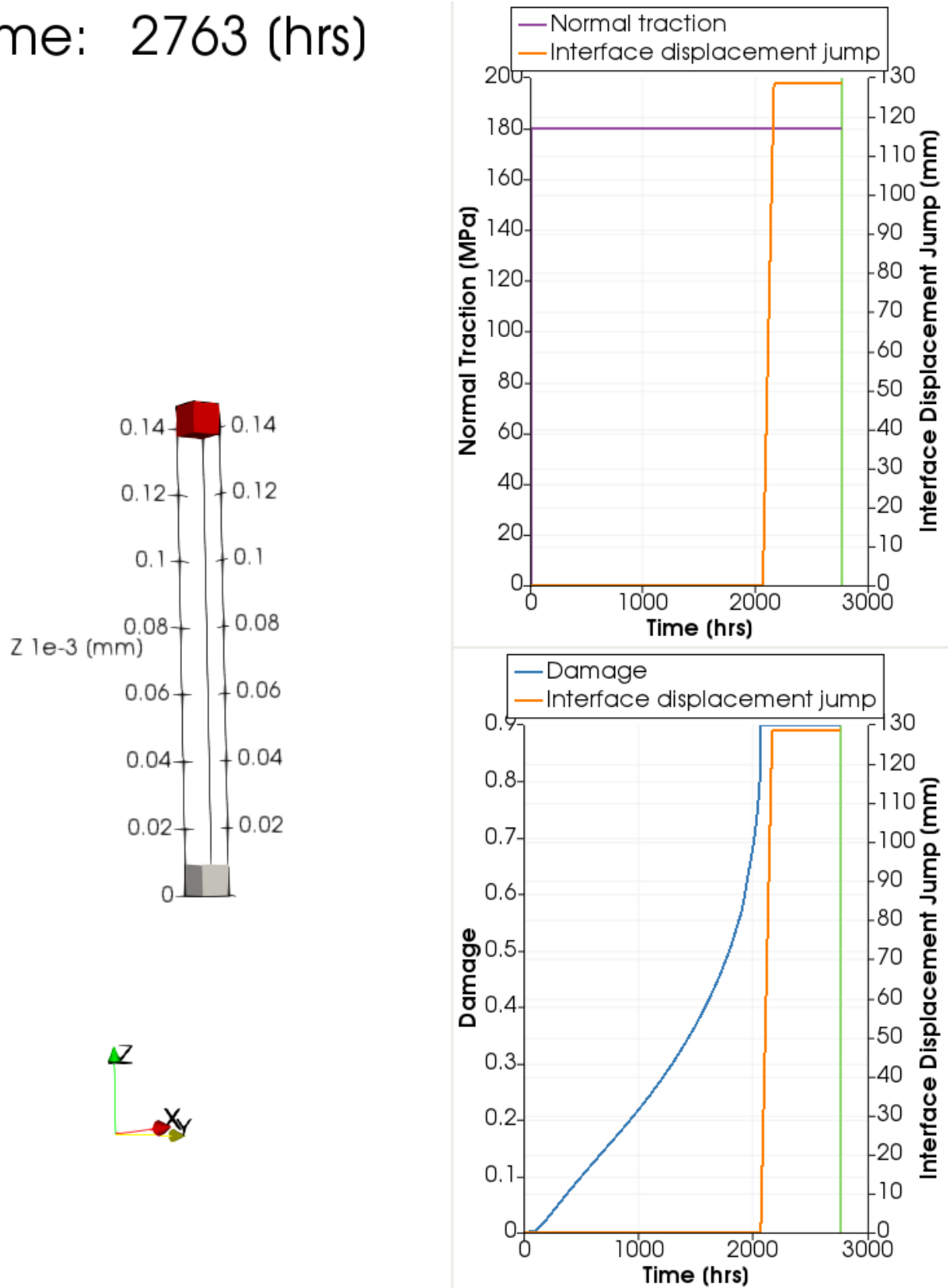

Figure 4.6: Results for the 2 element, stress-controlled simulation utilizing only the $\dot{V}_{L}$ term: a) final configuration after rupture (displacements are reduced by a factor of 1000), b) traction and the interface displacement jump versus time and c) the interface damage and the interface displacement jump versus time. 
Initial study of notch sensitivity of Grade 91 using mechanisms motivated crystal plasticity finite element method

September 2019

\section{Time: 2550 (hrs)}
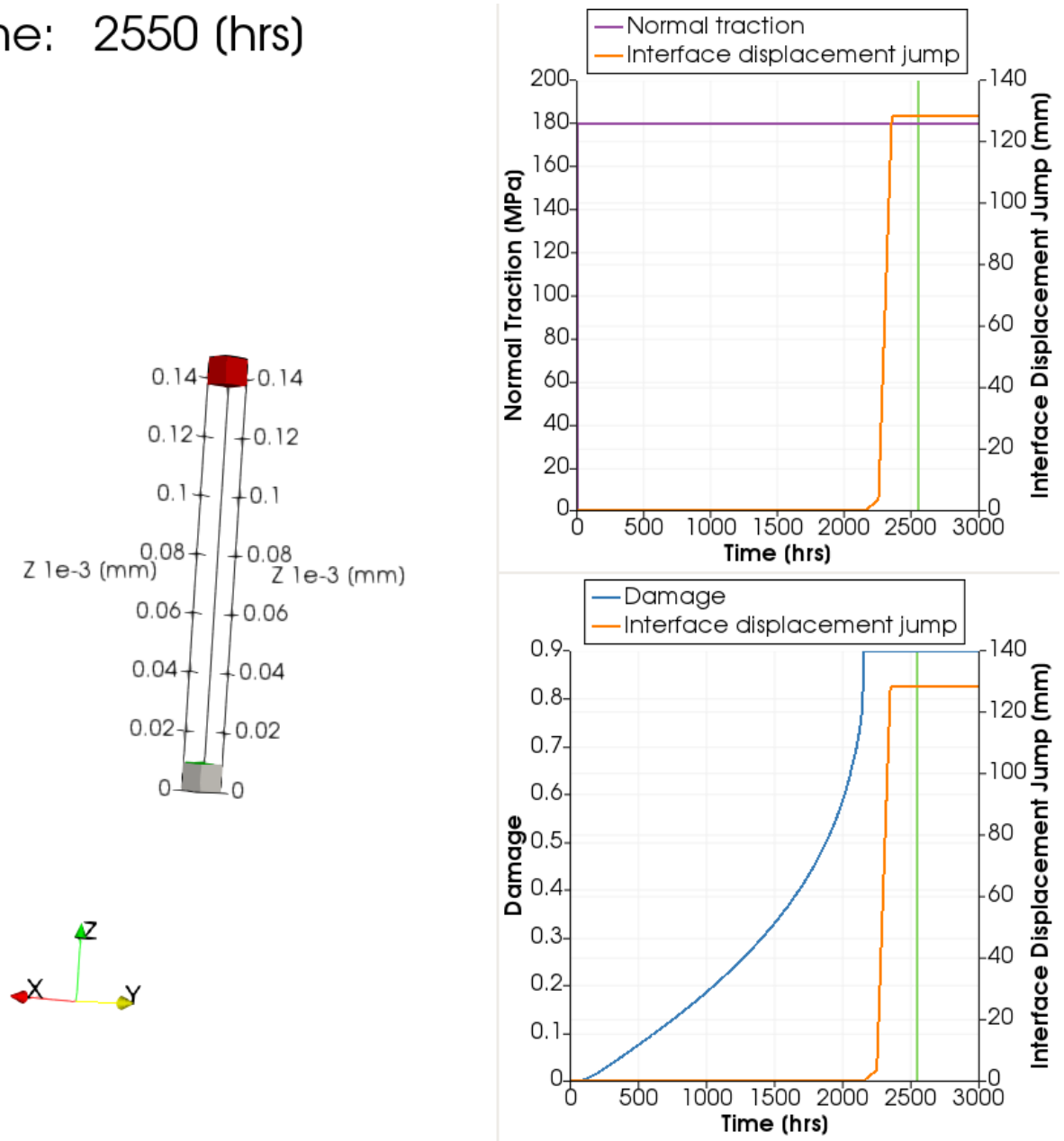

Figure 4.7: Results for the 2 element, stress-controlled simulation utilizing only the $\dot{V}_{H}$ term: a) final configuration after rupture (displacements are reduced by a factor of 1000), b) traction and the interface displacement jump versus time and c) the interface damage and the interface displacement jump versus time. 
Initial study of notch sensitivity of Grade 91 using mechanisms motivated crystal plasticity finite element method

September 2019

\section{Time: 2763 (hrs)}
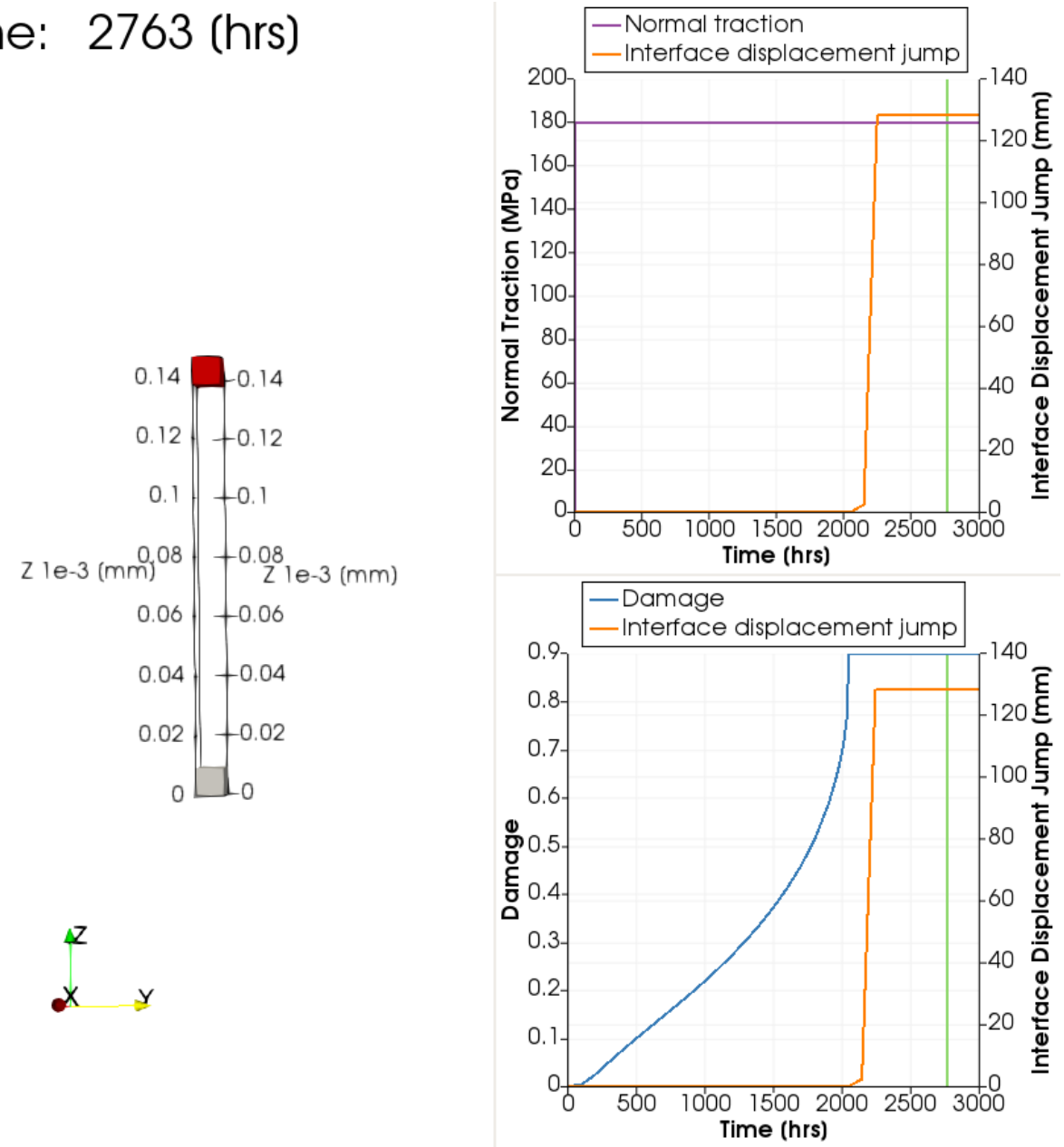

Figure 4.8: Results for the 2 element, stress-controlled simulation utilizing both $\dot{V}_{L}$ and $\dot{V}_{H}$ terms: a) final configuration after rupture (displacements are reduced by a factor of 1000), b) traction and the interface displacement jump versus time and c) the interface damage and the interface displacement jump versus time. 
Initial study of notch sensitivity of Grade 91 using mechanisms motivated crystal plasticity finite element method

September 2019

\begin{tabular}{lllllll} 
surface & disp $_{x}$ & disp $_{y}$ & disp $_{z}$ & $T_{x}$ & $T_{y}$ & $T_{z}$ \\
\hline$x=0$ & 0 & free & free & N/A & $N / A$ & $N / A$ \\
$x=X$ & equalvalue & free & free & 0 & $N / A$ & $N / A$ \\
\hline$y=0$ & free & 0 & free & $N / A$ & $N / A$ & $N / A$ \\
$y=Y$ & free & equalvalue & free & $N / A$ & 0 & $N / A$ \\
\hline$z=0$ & free & free & 0 & $N / A$ & $N / A$ & $N / A$ \\
$z=Z$ & free & free & equalvalue & $N / A$ & $N / A$ & 180
\end{tabular}

Table 4.6: Boundary conditions for the stress controlled, 2 element simulations, Traction values are in MPa.

the softening behavior exhibited by Grade 91 due to microstructural coarsening:

$$
\begin{array}{r}
\dot{\varepsilon}_{e q}^{C}=\dot{\varepsilon}_{0}\left(\frac{\sigma}{\eta}\right)^{n} \text { with } \\
\eta=\eta_{0}+A\left(1.0-\exp \left(-b \varepsilon_{e q}^{C}\right)\right)
\end{array}
$$

where $\varepsilon_{0}=1 \cdot 10^{-4} 1 / \mathrm{hr}$ is a scaling constant $\eta_{0}=220 \mathrm{MPa}$ is the initial fluidity, $A=-20$ is the maximum allowed fluidity variation, $b=50$ is parameter related to the rate of change of $\eta_{0}$, and $n=5$ is the power law creep exponent. The values $\varepsilon_{0}, n$, and $\eta_{0}$ were selected to match the value suggested in [34]. The aim of this test were two-fold: (i) provide a complex environment to assess the improved interface model and (ii) test the possibility of material softening to better describe the deformation mechanisms in Grade 91.

Results were not as expected. After approximately 11.000 model hours the simulations started having difficulties converging. We found that for some interface material points the behavior of the $\dot{V}_{H}$ equation becomes unreasonable. When damage starts accumulating (Fig. 4.9.a) the normal traction $T_{N}$ starts decreasing (Fig. 4.9.b), however rather than approaching zero in an asymptotic manner it plunges to unreasonable negative values (a few $G P a$ ), the interface continues to separate (Fig. 4.9.c ), and damage keeps increasing. This happens because the triaxial volumetric cavity growth term of $\dot{V}_{H}$ becomes very large and positive and thus overpowers the diffusion related cavity growth term. This produces a net positive cavity growth rate (Fig. 4.9.g and 4.10) under interface compression. This situation can only happen if the triaxility (4.9.e ) remains positive under compressive normal tractions. These physical conditions exist only if the interface is subjected to large positive transverse stresses (4.9.f). This unreasonable behavior results from the assumption that cavities have a fixed shape and that an increase in damage must be supported by an increase in separation. As already mentioned, the $\dot{V}_{H}$ term is responsible for including the in-plane void-void interaction effect representing cavity coalescence. However, it does so by increasing the interface separation rather than making cavities more oblate. In other words the failure of a ligament it is mathematically represented by generating a bigger spherical cavity, which is not a reasonable behavior. So we decided to drop $\dot{V}_{H}$ term. The $\dot{V}_{L}$ term behaves better because its triaxial contribution does not accelerate damage accumulation as fast the $\dot{V}_{H}$ term does. However there is no guarantee this behavior will not manifest under some other circumstance. Therefore we added a safeguard to the interface material point killing procedure (cf. Section 4.2.1.2). This safeguard tracks damage accumulation under negative 
Initial study of notch sensitivity of Grade 91 using mechanisms motivated crystal plasticity finite element method

September 2019
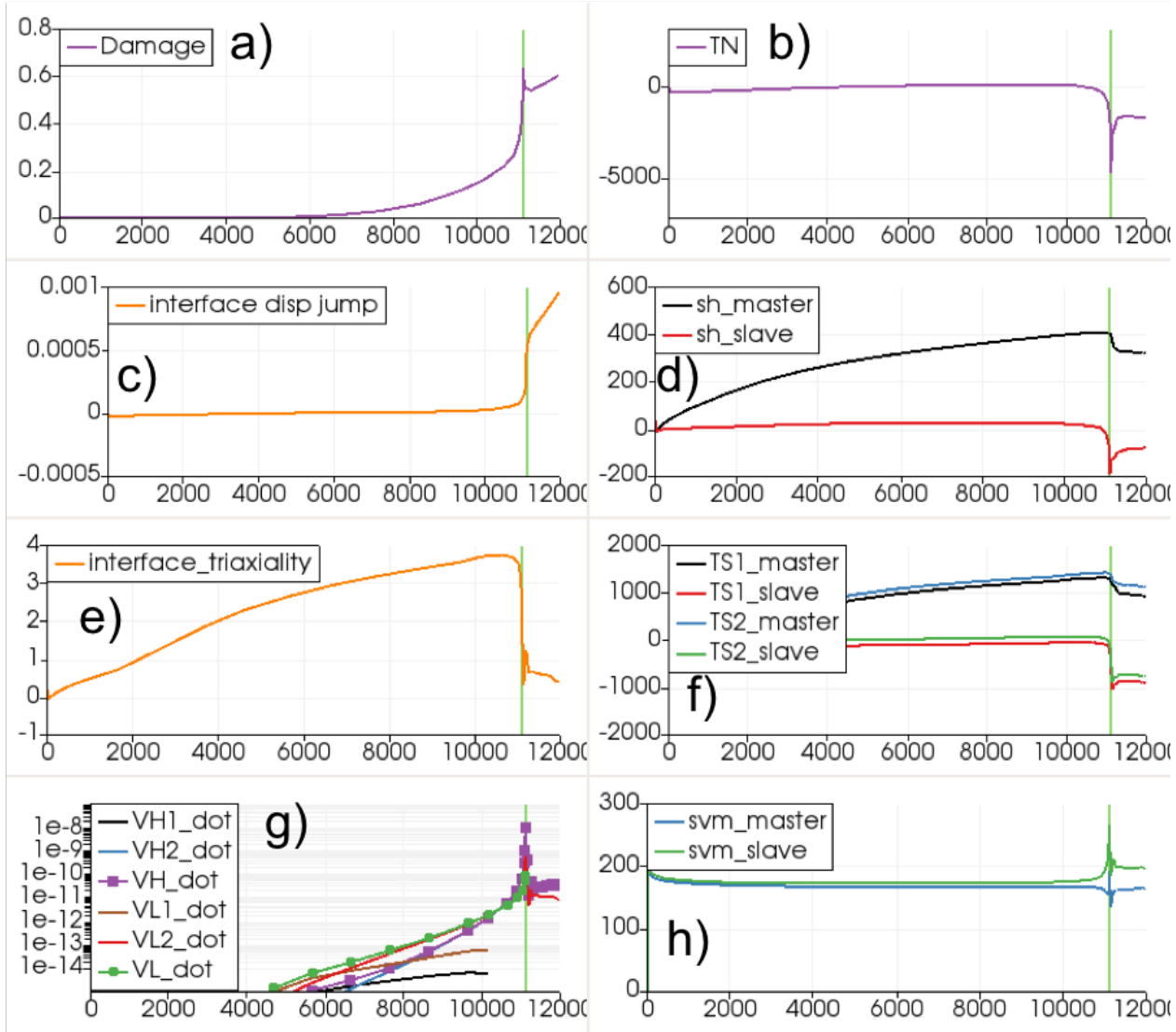

Figure 4.9: Detailed results for the interface quadrature point exhibiting a damage increase under compression: a) damage accumulation, b) normal traction, c) interface separation d) hydrostatic stress in adjacent bulk elements, e) interface triaxiality, f) transverse stresses in adjacent bulk elements, g) calculated cavity growth rate: $\dot{V}_{L}, \dot{V}_{H}$ and all their components, f) von Mises stresses in adjacent bulk elements.

tractions and marks the material point as failed if the damage accumulation rate and/or traction rate are too high. We believe this described behavior is one of the reasons why simulations performed in WARP3D were terminating before achieving reasonable values of creep ductility. The revised model corrects this numerical defect.

Figure 4.11 compares the global strain and strain rate of two simulations, one utilizing only $\dot{V}_{L}$ (solid lines) and the other using the maximum between $\dot{V}_{L}$ and $\dot{V}_{H}$ (squares). The latter simulation is the one in which the unreasonable behavior was found. The strains and strain rates of both simulation are comparable until the numerical instability arises. The simulation utilizing only the $\dot{V}_{L}$ term is able to capture tertiary creep up to very large strains.

The last test was to compare results obtained in MOOSE for different mesh refinements against both experimental [27] results and results obtained previously with WARP3D. We used NEPER [41] to generate a 100 grain random microstructure and used three different element sizes. The NEPER regularization features was used to remove small ("sliver") elements in the discretizations. We simulated a uniaxial creep test at $140 \mathrm{MPa}$ for the different meshes and compared the results. The average characteristic element length of the 
Initial study of notch sensitivity of Grade 91 using mechanisms motivated crystal plasticity finite element method

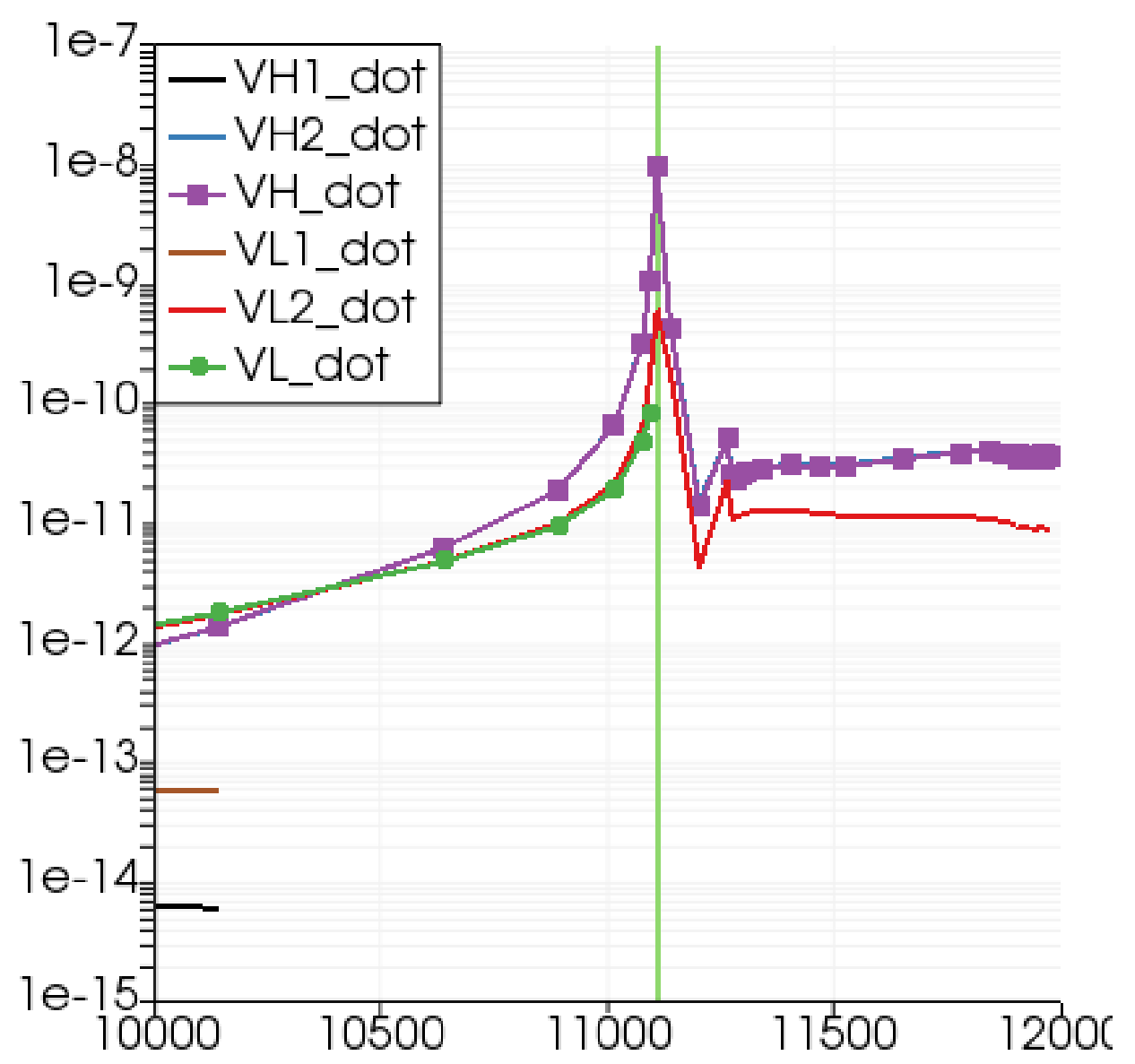

Figure 4.10: Cavity growth rates $\dot{V}_{L}$ and $\dot{V}_{H}$ and all their components before failure. 
Initial study of notch sensitivity of Grade 91 using mechanisms motivated crystal plasticity finite element method
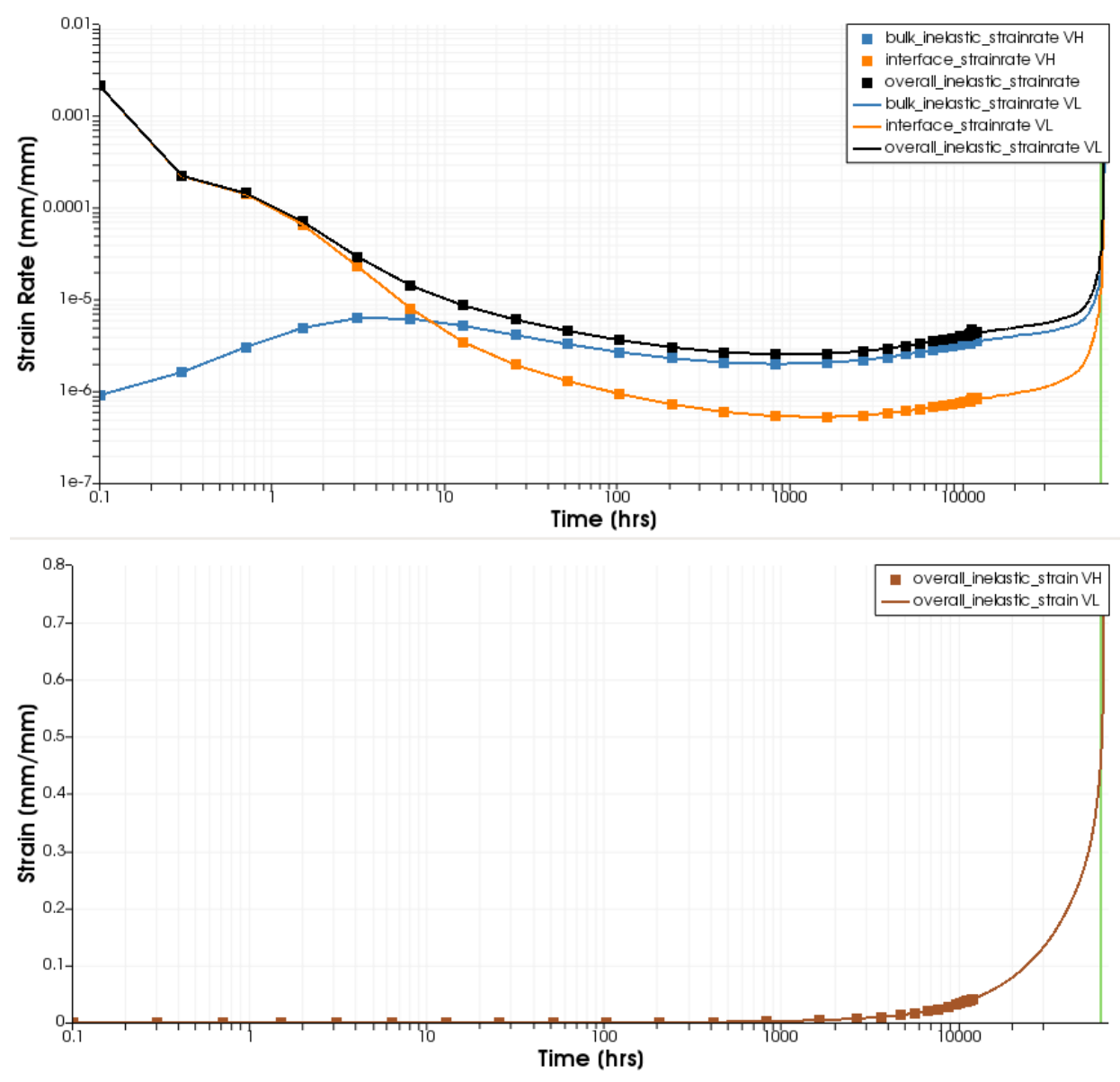

Figure 4.11: Comparison of global strains and strain rates for two different simulations, one using both volumetric growth rate terms $\left(\dot{V}_{L}\right.$ a and $\dot{V}_{H}$, squares $)$ and the other using only the $\dot{V}_{L}$ term (solid lines). a) Comparison of the strain rates versus time. The individual contributions of the interface and grain rates to the overall strain rate is also shown. b) Overall strain for both simulations. 
Initial study of notch sensitivity of Grade 91 using mechanisms motivated crystal plasticity finite element method

September 2019

14,000 and 10,000 elements meshes are respectively smaller and larger than the characteristic element length used in WARP3D mesh. The WARP3D simulations apply true periodic mesh and boundary conditions, while the MOOSE simulations use a set of symmetry planes and equal value boundary constraints to emulate periodic boundary conditions (see Table 4.8 for details). The MOOSE simulations used the new crystal model implemented in NEML with a Voce hardening model that includes the diffusion contribution resolved to each slip system. The parameters of the model are presented in Table 4.7.

Results are shown in Figure 4.12. The various simulations reach different final creep strains because we allocated the same wall-time to all of them, and not because they fail at difference physical times. The behavior of all simulations is similar up to the onset of tertiary creep. The time to tertiary is clearly mesh dependent however the variation between the coarser and the finer mesh is still in the range of the experimental scatter. Furthermore, results correctly capture the experimental trend shown by Kimura et al. [27] for the same conditions.

\begin{tabular}{llll} 
symbol & description & value & units \\
\hline$\tau_{0}$ & initial resolved critical shear stress & 40 & $\mathrm{MPa}$ \\
\hline$\tau_{\text {sat }}$ & saturation stress & 12 & $\mathrm{MPa}$ \\
\hline$b$ & voce hardening paramter & 66.67 & unitless \\
\hline$\gamma_{0}$ & scaling constant & $9.55470706737 \cdot 10^{-8}$ & $1 / \mathrm{MPa}$ \\
\hline$n$ & hardening exponent & 1 & unitless \\
\hline$A$ & diffusion creep parameter & $1.2 \cdot 10^{-9}$ & $1 / \mathrm{MPa}$ \\
\hline$n_{\text {diff }}$ & diffusion creep exponent & $1 \cdot 10^{-9}$ & unitless \\
\hline$E$ & young modulus & $150 \cdot 10^{3}$ & $\mathrm{MPa}$ \\
\hline$\nu$ & Poisson's ratio & 0.285 & unitless \\
\hline
\end{tabular}

Table 4.7: CPFEM simulation parameters.

\begin{tabular}{lllllll} 
surface & disp $_{x}$ & disp $_{y}$ & disp $_{z}$ & $T_{x}$ & $T_{y}$ & $T_{z}$ \\
\hline$x=0$ & 0 & free & free & N/A & N/A & N/A \\
$x=X$ & equalvalue & free & free & 0 & N/A & N/A \\
\hline$y=0$ & free & 0 & free & N/A & N/A & N/A \\
$y=Y$ & free & equalvalue & free & N/A & 0 & N/A \\
\hline$z=0$ & free & free & 0 & $N / A$ & $N / A$ & $N / A$ \\
$z=Z$ & free & free & equalvalue & $N / A$ & $N / A$ & 140
\end{tabular}

Table 4.8: Boundary conditions for the stress controlled, 2 element simulations. Traction values are in $\mathrm{MPa}$.

At this point we consider the small deformation implementation of the grain boundary cavitation model complete. The only tasks remaining are to include large deformations (area changes and rotations) in the CZM and to allow the use of true periodic boundary conditions with the CZM. Furthermore, these results highlight the need to improve the GB cavitation model by allowing cavities to become oblate. 
Initial study of notch sensitivity of Grade 91 using mechanisms motivated crystal plasticity finite element method

Inelastic Strain Vs Time

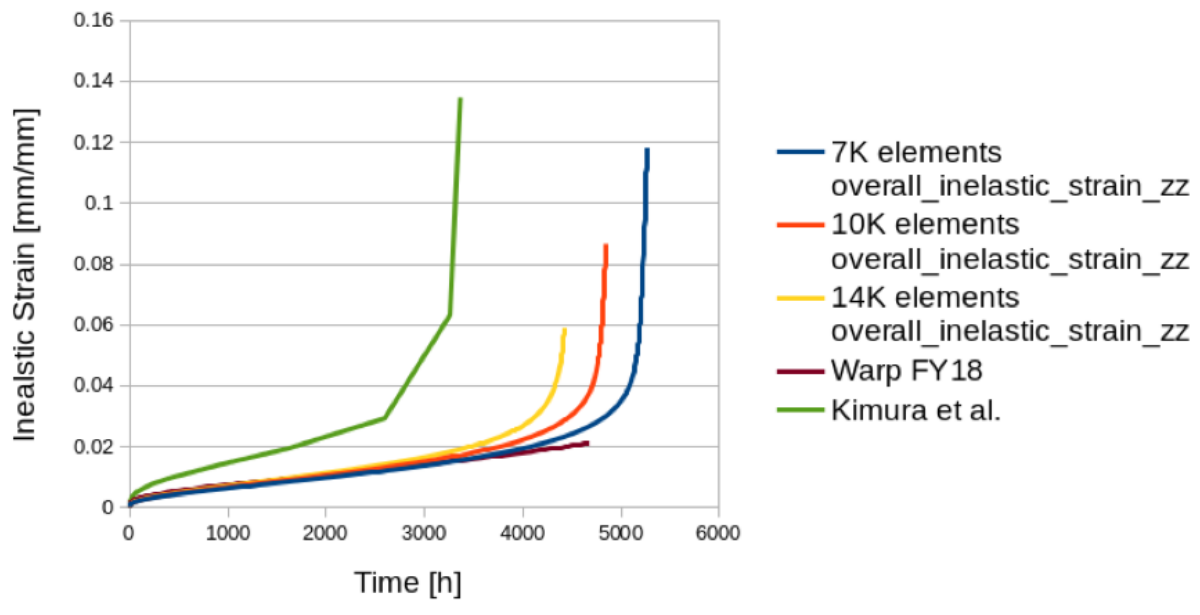

Figure 4.12: Mesh refinement study results. 

Initial study of notch sensitivity of Grade 91 using mechanisms motivated crystal plasticity finite element method

September 2019

\section{Effective stress measure for triaxial loading}

For high temperature applications effective stresses are used to correlate a general stress state to the time to rupture measured under uniaxial stress conditions. This correlation is usually achieved with some linear or nonlinear combination of stress invariant that can be formulated in terms of principal stresses. One of the simplest effective stress measure is the maximum principal stress $\sigma_{1}$ (with $\sigma_{1} \geq \sigma_{2} \geq \sigma_{3}$ ) Other effective stress measures can be defined with a combination of the principal stresses. For instance the von Mises can be expressed as

$$
\sigma_{V M}=\sqrt{\frac{1}{2}\left[\left(\sigma_{1}-\sigma_{2}\right)^{2}+\left(\sigma_{1}-\sigma_{3}\right)^{2}+\left(\sigma_{2}-\sigma_{3}\right)^{2}\right]}
$$

The von Mises stress is strictly related to the second deviatoric stress invariant by:

$$
\sigma_{V M}=\sqrt{3 J_{2}}
$$

By definition the $\sigma_{V M}$ only retains the deviatoric portion of the stress tensor, thus neglecting mean stress effect. The first invariant of the stress is

$$
I_{1}=\sigma_{1}+\sigma_{2}+\sigma_{3}
$$

and the hydrostatic stress is

$$
\sigma_{H}=\frac{I_{1}}{3}
$$

By using a combination of some of the above stress invariants Hayhurst [20] proposed the following effective stress that combines the von Mises, hydrostatic, and maximum principal stress :

$$
\sigma_{e}=\alpha\left\langle\sigma_{1}\right\rangle+\beta I_{1}+\gamma \sigma_{V M} \text { with } \alpha+\beta+\gamma=1
$$

where $\alpha, \beta$, and $\gamma$ are material dependent parameters that need to be calibrated with experimental data and are bounded such they are greater than zero.

In a later work Hayhurst and Henderson [22] suggested that the role of the first stress invariant $I_{1}$ on creep rupture time is already accounted for by including the first principal stress and the von Mises stress. Therefore, they proposed a simplified effective stress:

$$
\sigma_{e}=\alpha\left\langle\sigma_{1}\right\rangle+\gamma \sigma_{V M} \text { with } \alpha+\gamma=1
$$

where $\alpha$ and $\gamma$ are material dependent parameters that needs to be calibrated with experimental data and are bounded between 0 and 1 . The symbol $\langle\cdot\rangle$ means that only the positive part of the argument is retained. Huddleston [24] proposed a different effective stress measure that is a nonlinear combination of the von Mises stress and principal stresses:

$$
\sigma_{e}=\sigma_{V M} \exp \left[b\left(\frac{I_{1}}{\sqrt{\sigma_{1}^{2}+\sigma_{2}^{2}+\sigma_{3}^{2}}}\right)\right]
$$

where $b$ is a parameters that needs to be calibrated with multiaxial experimental results. The parameter $b$ is not bounded. The Huddleston effective stress is currently used by Section III, Division 5 of the ASME Boiler and Pressure Vessel design code [2] and by the British fitness for service manual R5 [14]. The French design code RCC-MRx [1] provides two different 
Initial study of notch sensitivity of Grade 91 using mechanisms motivated crystal plasticity finite element method

September 2019

options to compute the effective stress, one based on von Mises stress (Eq. 5.8) and the other maximum shear (Eq. 5.9). In both cases the code suggests the user accounts for the first stress invariant if data are available.

$$
\begin{aligned}
& \sigma_{e}=\alpha \sigma_{V M}+\beta I_{1} \alpha+\beta=1 \\
& \sigma_{e}=\alpha\left(\sigma_{1}-\sigma_{3}\right)+\beta I_{1} \text { with } \alpha+\beta=1
\end{aligned}
$$

where $\alpha$ and $\beta$ are a material dependent parameters that need to be calibrated with experimental data and are bounded between 0 and 1 .

The calibration of effective stress parameters is usually performed with uniaxial and, if available, biaxial experimental results. The calibrated effective stress is then used by designer to estimate the creep rupture time of a component. Historically, biaxial test data was sufficient because conventional reactors often utilize thin wall structure for components subject to creep. However, as discussed previously, the core block of a heat pipe microreactor generates substantially triaxial stresses. Therefore, this section assesses these common effective stress measures for triaxial stress states. However, to the best of our knowledge, triaxial creep tests with controlled stresses have never been performed. Therefore, this chapter uses the validated physically-based model to assess the effective stress measures, in lieu of direct testing. A physically-based model can be used to extrapolate results outside its calibration envelope as long as the physics does not change. Such models can also provide results for conditions that are not experimentally achievable, either due to time or cost constraints. The improved model presented in Chapters 3 and 4 is already capable of reproducing available experimental results for Grade 91 (see Messner et al. [34] and Messner et al. [37]) and so reasonably could be extended to multiaxial loads.

In this chapter we will use the model to generate creep rupture times for different multiaxial stress conditions. The data will be used to check if: (i) any of the effective stress measure presented above can be safely used to predict the creep rupture time under a generic multiaxial stress state and (ii) if there is a need to identify a way to produce triaxial experimental results. This chapter is organized as follows: first we will present the simulations used to generate triaxial rupture data, then we will present the procedures used to calibrate effective stress parameters, and finally we will compute the correlations and error of each stress measure against the results of the full CPFEM calculations.

\subsection{Simulations setup and rupture time}

Four increments of stress were used to generate loading combinations: 0, 60, 100, 140, and $180 \mathrm{MPa}$. The multiaxial stress space was probed by considering all possible combinations of these stress values assigned to each of the three principal stresses. Table 5.1 list all the simulations used to generate creep rupture time data in terms of the principal stresses. For each simulation, each principal stress was applied as a constant load (dead load) condition on one face of a periodic RVE. Pure triaxial stress state simulations (e.g. $\left.\sigma_{1}=\sigma_{2}=\sigma_{3}\right)$ ) were performed but are not included in the results as they do not produce reasonable rupture times.

To compute a consistent time to rupture we had to select a failure criterion. Requiring all the simulation to run until complete failure was ruled out because of the computational 
Initial study of notch sensitivity of Grade 91 using mechanisms motivated crystal plasticity finite element method

September 2019

\begin{tabular}{|c|c|c|c|c|c|}
\hline stress state & $\begin{array}{l}\sigma_{1} \\
(\mathrm{MPa})\end{array}$ & $\begin{array}{l}\sigma_{2} \\
(\mathrm{MPa})\end{array}$ & $\begin{array}{l}\sigma_{3} \\
(\mathrm{MPa})\end{array}$ & $\begin{array}{l}\text { Triaxiality } \\
(-)\end{array}$ & $\begin{array}{l}\text { Time to rupture } \\
\text { (hours) }\end{array}$ \\
\hline uniaxial & 60 & 0 & 0 & 0.33 & $3.04 \mathrm{E}+05$ \\
\hline uniaxial & 100 & 0 & 0 & 0.33 & $4.55 \mathrm{E}+04$ \\
\hline uniaxial & 140 & 0 & 0 & 0.33 & $4.63 \mathrm{E}+03$ \\
\hline uniaxial & 180 & 0 & 0 & 0.33 & $1.10 \mathrm{E}+03$ \\
\hline biaxial & 60 & 60 & 0 & 0.67 & $3.11 \mathrm{E}+05$ \\
\hline biaxial & 100 & 60 & 0 & 0.61 & $6.95 \mathrm{E}+04$ \\
\hline biaxial & 140 & 60 & 0 & 0.55 & $9.76 \mathrm{E}+03$ \\
\hline biaxial & 180 & 60 & 0 & 0.50 & $1.45 \mathrm{E}+03$ \\
\hline biaxial & 100 & 100 & 0 & 0.67 & $4.85 \mathrm{E}+04$ \\
\hline biaxial & 140 & 100 & 0 & 0.64 & $9.11 \mathrm{E}+03$ \\
\hline biaxial & 180 & 100 & 0 & 0.60 & $1.55 \mathrm{E}+03$ \\
\hline biaxial & 140 & 140 & 0 & 0.67 & $5.16 \mathrm{E}+03$ \\
\hline biaxial & 180 & 140 & 0 & 0.65 & $1.41 \mathrm{E}+03$ \\
\hline biaxial & 180 & 180 & 0 & 0.67 & $1.15 \mathrm{E}+03$ \\
\hline triaxial & 100 & 60 & 60 & 1.83 & $1.79 \mathrm{E}+05$ \\
\hline triaxial & 140 & 60 & 60 & 1.08 & $4.78 \mathrm{E}+04$ \\
\hline triaxial & 180 & 60 & 60 & 0.83 & $7.22 \mathrm{E}+03$ \\
\hline triaxial & 100 & 100 & 60 & 2.17 & $1.71 \mathrm{E}+05$ \\
\hline triaxial & 140 & 100 & 60 & 1.44 & $5.84 \mathrm{E}+04$ \\
\hline triaxial & 180 & 100 & 60 & 1.07 & $1.15 \mathrm{E}+04$ \\
\hline triaxial & 140 & 140 & 60 & 1.42 & $4.69 \mathrm{E}+04$ \\
\hline triaxial & 180 & 140 & 60 & 1.20 & $1.20 \mathrm{E}+04$ \\
\hline triaxial & 180 & 180 & 60 & 1.17 & $1.20 \mathrm{E}+04$ \\
\hline triaxial & 140 & 100 & 100 & 2.83 & $1.00 \mathrm{E}+05$ \\
\hline triaxial & 180 & 100 & 100 & 1.58 & $2.57 \mathrm{E}+04$ \\
\hline triaxial & 140 & 140 & 100 & 3.17 & $8.26 \mathrm{E}+04$ \\
\hline triaxial & 180 & 140 & 100 & 2.02 & $3.35 \mathrm{E}+04$ \\
\hline triaxial & 180 & 180 & 100 & 1.92 & $2.76 \mathrm{E}+04$ \\
\hline triaxial & 180 & 140 & 140 & 3.83 & $5.05 \mathrm{E}+04$ \\
\hline triaxial & 180 & 180 & 140 & 4.17 & $4.67 \mathrm{E}+04$ \\
\hline
\end{tabular}

Table 5.1: Stress states imposed for the different simulations classified by stress state. Triaxility and calculated time to rupture are also reported. Horizontal lines group simulations into uniaxial, biaxial and triaxial groups. 
Initial study of notch sensitivity of Grade 91 using mechanisms motivated crystal plasticity finite element method

September 2019

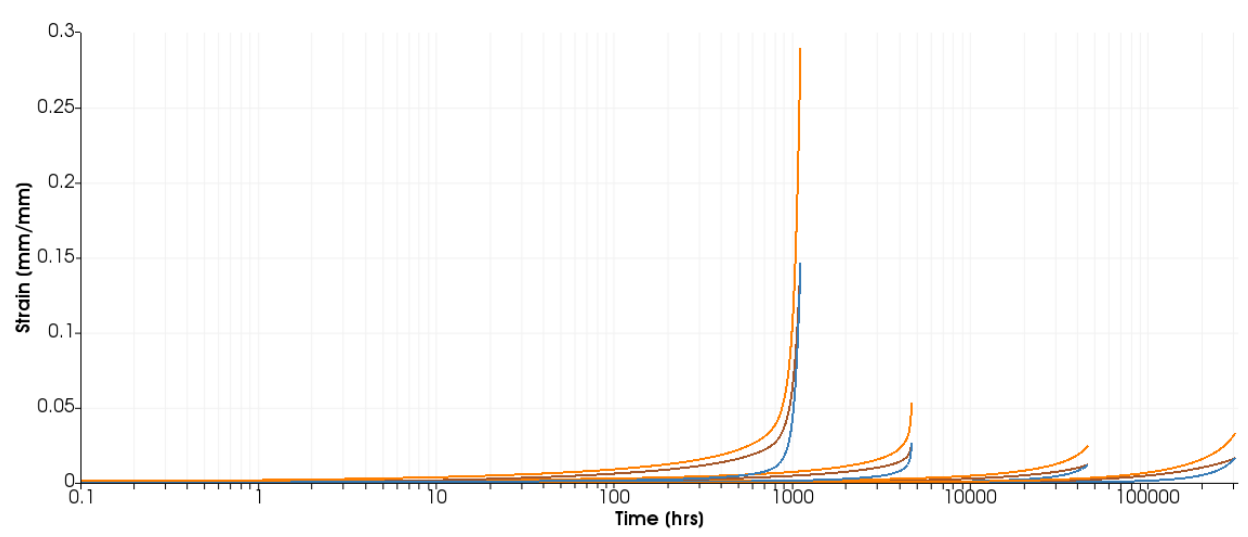

Figure 5.1: Cell average equivalent strain versus time (orange) for uniaxial simulations. The interface equivalent strain (blue) and the grain equivalent inelastic strain (brown) are also depicted. Stress level decreases from left to right from $180 \mathrm{MPa}$ to $60 \mathrm{MPa}$

cost, as small substeps are required to process the last few percent creep strain. The time to achieve a certain strain was also ruled out because the target strain must be known a-priori but creep ductility is a function of the applied stress. Therefore we decided to declare failure when the equivalent interface strain becomes equal to the equivalent inelastic bulk strain. Figure 5.1 depicts the total cell equivalent strain (orange) versus time up to rupture for the simulated uniaxial stress cases. The interface (blue) and grain bulk (brown) inelastic equivalent strains contributions are also depicted. The trend of reduced creep ductility for smaller values of applied uniaxial stress is supported by experimental evidence.

\subsection{Effective stresses calibration}

To compute the time to rupture from an effective stress first a uniaxial time-stress relationship must be defined. Often, the time to rupture can be expressed as a function of an effective stress using a simple power law creep model such as:

$$
t_{R}=A\left(\sigma_{e}\right)^{n}
$$

where $A$ and $n$ are material parameters. By construction an effective stress must be equivalent to the applied stress for uniaxial loading conditions, so uniaxial test cases will be use to calibrate $A$ and $n$. Moreover, $A$ and $n$ are material parameters and should be independent from the effective stress measure. Therefore here we calibrate the rupture correlation parameters against the uniaxial data only and then apply that uniaxial correlation to predict multiaxial failure. This is the approach used in current high temperature design methods. Previous work with this CPFEM model demonstrates a mechanism shift at lower values of stress [37]. For the current work we want to use a uniaxial rupture correlation that captures this mechanism shift. As such we elected to use a piecewise log-linear relation to capture both the high stress and low stress power law relations between uniaxial stress and rupture. With this choice the uniaxial rupture time is perfectly predicted by all the effective stresses. The only differences will therefore be in the ability of the different stress measures to predict 
Initial study of notch sensitivity of Grade 91 using mechanisms motivated crystal plasticity finite element method

September 2019

multiaxial rupture. The identified values $A$ and $n$ and associated stress ranges are presented in Table 5.2

\begin{tabular}{lll} 
stress range & $\mathrm{A}$ & $\mathrm{n}$ \\
\hline$\sigma_{e}<100$ & $1.24915179 e+12$ & -3.71914119 \\
$100 \leq \sigma_{e}<140$ & $1.76058693 e+18$ & -6.79366232 \\
$\sigma_{e} \geq 140$ & $8.37396380 e+15$ & -5.71137536
\end{tabular}

Table 5.2: Identified values of $A$ and $n$ for different stress ranges (Eq. 5.10).

\subsection{Effective stresses parameters calibration}

The analyzed effective stress measures are summarized in Table 5.3.

$\begin{array}{ll}\text { name } & \text { equation } \\ \text { max } & \sigma_{1} \\ \text { Hayhurst_1972 } & \alpha\left\langle\sigma_{1}\right\rangle+\beta I_{1}+\gamma \sigma_{V M} \\ \text { HLM_1978 } & \alpha\left\langle\sigma_{1}\right\rangle+\gamma \sigma_{V M} \\ \text { huddleston } & \sigma_{V M} \exp \left[b\left(\frac{I_{1}}{\sqrt{\sigma_{1}^{2}+\sigma_{2}^{2}+\sigma_{3}^{2}}}\right)\right] \\ \text { RCC-MRx_mises } & \alpha \sigma_{V M}+\beta I_{1} \\ \text { RCC-MRx_max } & \alpha\left(\sigma_{1}-\sigma_{3}\right)+\beta I_{1}\end{array}$

Table 5.3: List of equations for the analyzed effective stresses.

The parameters of each effective stress (for those that have free parameters) have been calibrated utilizing a best fit approach by minimizing the total root mean square relative error (RMRSE) of the predicted rupture time for the effective stress versus the rupture computed via CPFEM simulations. The RMSRE for the optimization problem was defined as:

$$
R M S R E=\sqrt{\frac{1}{N} \sum_{i=1}^{N}\left(\frac{t_{e f f, i}-t_{c p, i}}{t_{c p, i}}\right)^{2}}
$$

where $t_{c p}$ is the time to rupture computed via CPFEM simulations, $t_{e f f}$ is the time to rupture predicted by the effective stress, $N$ is the number of data points and $i$ is the index indicating a particular datapoint. 
Initial study of notch sensitivity of Grade 91 using mechanisms motivated crystal plasticity finite element method

September 2019

The effective stress parameters have been calibrated twice, once utilizing uniaxial and biaxial data, and once utilizing all the available datapoints, including triaxial stresses. The former procedure is representative of current design practice, as triaxial creep data is not available. Parameter calibration has been performed with both a global and a gradient based optimizer. For effective stress models having two variables 100 different initial starting point were utilized to ensure the fit finds a global minimum. For each variable initial guesses were distributed on a regular grid. The parameter $b$ in the Huddleston model is unbounded. In this case we used 20 initial regularly spaced initial guesses between 0 and 2.5. The parameter generating the smallest RMSRE are presented in Table 5.4

\begin{tabular}{llllll} 
effective stress name & calibration type & $\alpha$ & $\beta$ & $\gamma$ & $\mathrm{b}$ \\
\hline Hayhurst_1972 & biaxial & 0.538 & 0.00 & 0.461 & - \\
HLM_1978 & biaxial & 0.538 & - & 0.461 & - \\
huddleston & biaxial & - & - & - & 0.0627 \\
RCC-MR__mises & biaxial & 0.0282 & 0.971 & - & - \\
RCC-MRx_max & biaxial & 0.491 & 0.508 & - & - \\
\hline & & & & & \\
Hayhurst_1972 & triaxial & 0.409 & $9.86 \mathrm{e}-13$ & 0.590 & - \\
HLM_1978 & triaxial & 0.409 & - & 0.461 & - \\
huddleston & triaxial & - & - & - & 1.06 \\
RCC-MRx_mises & triaxial & 0.128 & 0.871 & - & - \\
RCC-MR_max & triaxial & 0.491 & 0.508 & - & -
\end{tabular}

Table 5.4: List of best fit parameters for all effective stresses.

\subsection{Results}

The root mean square error between the computed and predicted time rupture are show below for three conditions:

1. biaxial fit and RSMRE value computed considering only uniaxial and biaxial data

2. biaxial fit and RSMRE value computed considering all datapoints

3. triaxial fit and RSMRE value computed considering all datapoints

Error measure 1 assesses the measures for the current method of fitting and testing against biaxial experimental data. Error measure 2 assesses how an effective stress fit to the current, biaxial experimental data might perform when called upon to predict rupture for triaxial stress states. Error measure 3 assesses how the measures would perform in the best case where triaxial data is available, essentially selecting the best possible effective stress measure for arbitrary stress states.

Figure 5.2 compares the RSMRE errors for case 1 and case 2. Figure 5.2 compares the RSMRE errors for case 2 and case 3. Figures 5.4, 5.5, 5.6, 5.7, 5.8, 5.9 and 5.10 depict the distribution of RSMRE error for each effective stress for all the three cases above. 
Initial study of notch sensitivity of Grade 91 using mechanisms motivated crystal plasticity finite element method

September 2019

Results show that when parameters are calibrated against biaxial data, all the models can accurately predict the time to rupture for biaxial stress states. However, when calibrated against biaxial data only a few effective stress measures are able to reasonably predict the creep rupture life for triaxial stress states. Surprisingly the model proposed by Huddleston [24] and utilized by American Society of Mechanical Engineers [2] and EDF Energy Nuclear Generation Ltd [14] shows worst extrapolation behavior. The best models in terms of RSMRE are the ones proposed by Hayhurst et al (e.g. Hayhurst_1972, HLM_1978). They perform well when utilizing only biaxial data to predict the life for triaxial stress states (RSRME $45 \%$ ). Note that the optimizer sends parameter $\beta$ to zero for the Hayhurst_1972 model, degenerating it to the HLM_1978, in line with Hayhurst's observations.

Most of the models improve their prediction if calibrated against triaxial data but again the Hayhurst_1972 and HLM_1978 models are the most accurate. Again the optimizer degenerates the Hayhurst_1972 model to the HLM_1978 model, implying that a combination of von Mises and max principal stresses are sufficient and a dependence on the hydrostatic stress is not required.

The RSMRE error is not the only quantity that we need to consider when selecting an effective stress measure. Ideally, the error in the rupture life predictions using an effective stress measure would be on the conservative side, i.e. the effective stress predicts a shorter rupture life than the actual multiaxial rupture time. Considering this additional criteria and looking at the distribution of the RSMRE errors for case 2 (Figs,5.4-5.9.b ), note that max, hayhurst_1972, HLM_1978, and rccmrx_principal are conservative. Among the conservative effective stresses the most accurate are hayhurst_1972 and HLM_1978, which again the optimizer degenerates to be the same model, and the most (over) conservative are max and rccmrx_principal.

When calibrating against triaxial data all the models with parameters become conservative with the most conservative being rccmrx_principal and huddleston. The most accurate are again the hayhurst_1972 and HLM_1978 models, again both degenerating to the same, simple linear combination of the von Mises and maximum principal stresses.

These results suggest the use of either hayhurst_1972, HLM_1978 models to predict the creep rupture life of Grade 91 under multiaxial stress states. The data also suggests that care should be used when using effective stress measures calibrated against biaxial test data when predicting the creep rupture life for triaxial stress states as some of the commonly used effective stress measures are non-conservative. As discussed in Chapter 6, this conclusions should be validated experimentally. 
Initial study of notch sensitivity of Grade 91 using mechanisms motivated crystal plasticity finite element method

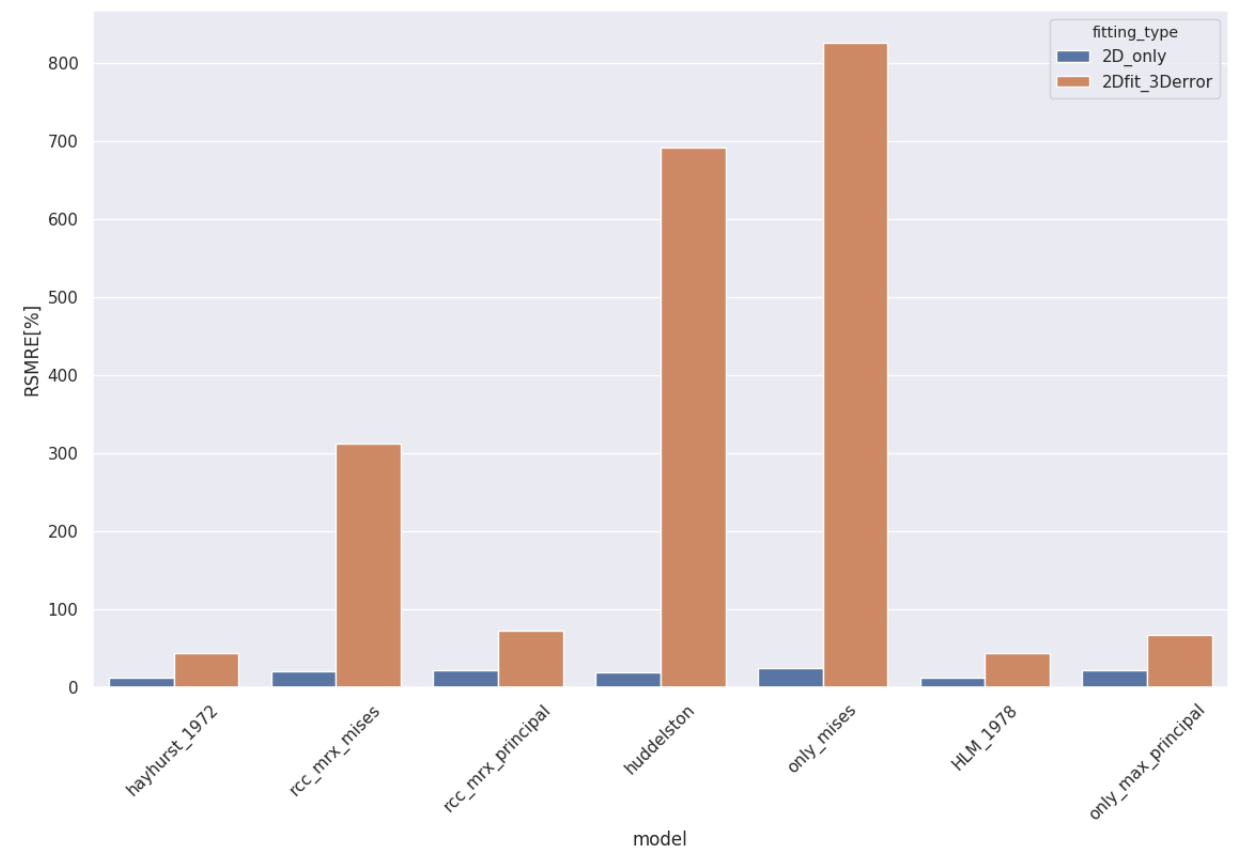

Figure 5.2: RMSRE of effective stress parameters calibrated against uniaxial and biaxial data versus the predicted time to rupture for the different effective stress models. Blue bars represent the RSMRE when only considering biaxial data, orange bars when considering all datapoints.

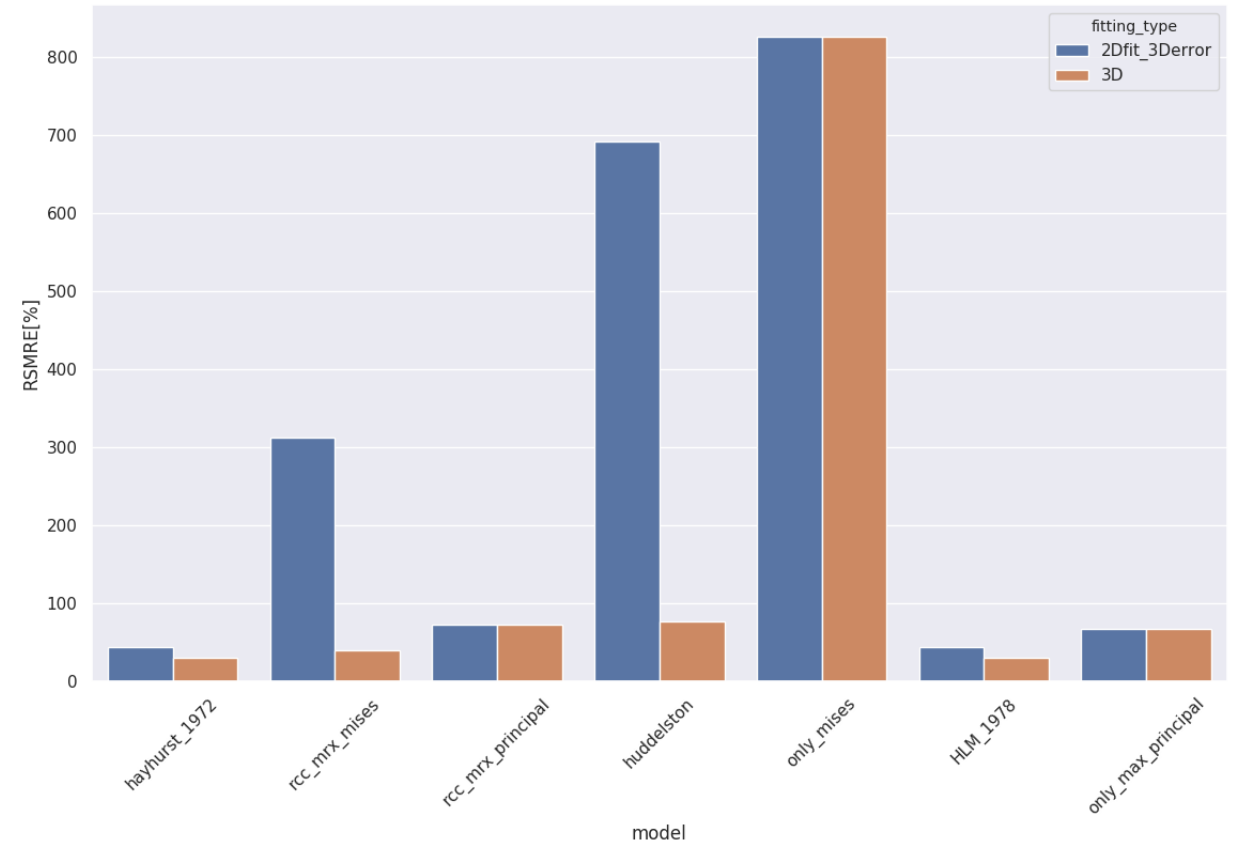

Figure 5.3: Comparison of RMSRE for effective stress parameters calibrated against: (i) uniaxial and biaxial data (blue) and (ii) all datapoints (orange). The error is computed by using all data points 
Initial study of notch sensitivity of Grade 91 using mechanisms motivated crystal plasticity finite element method
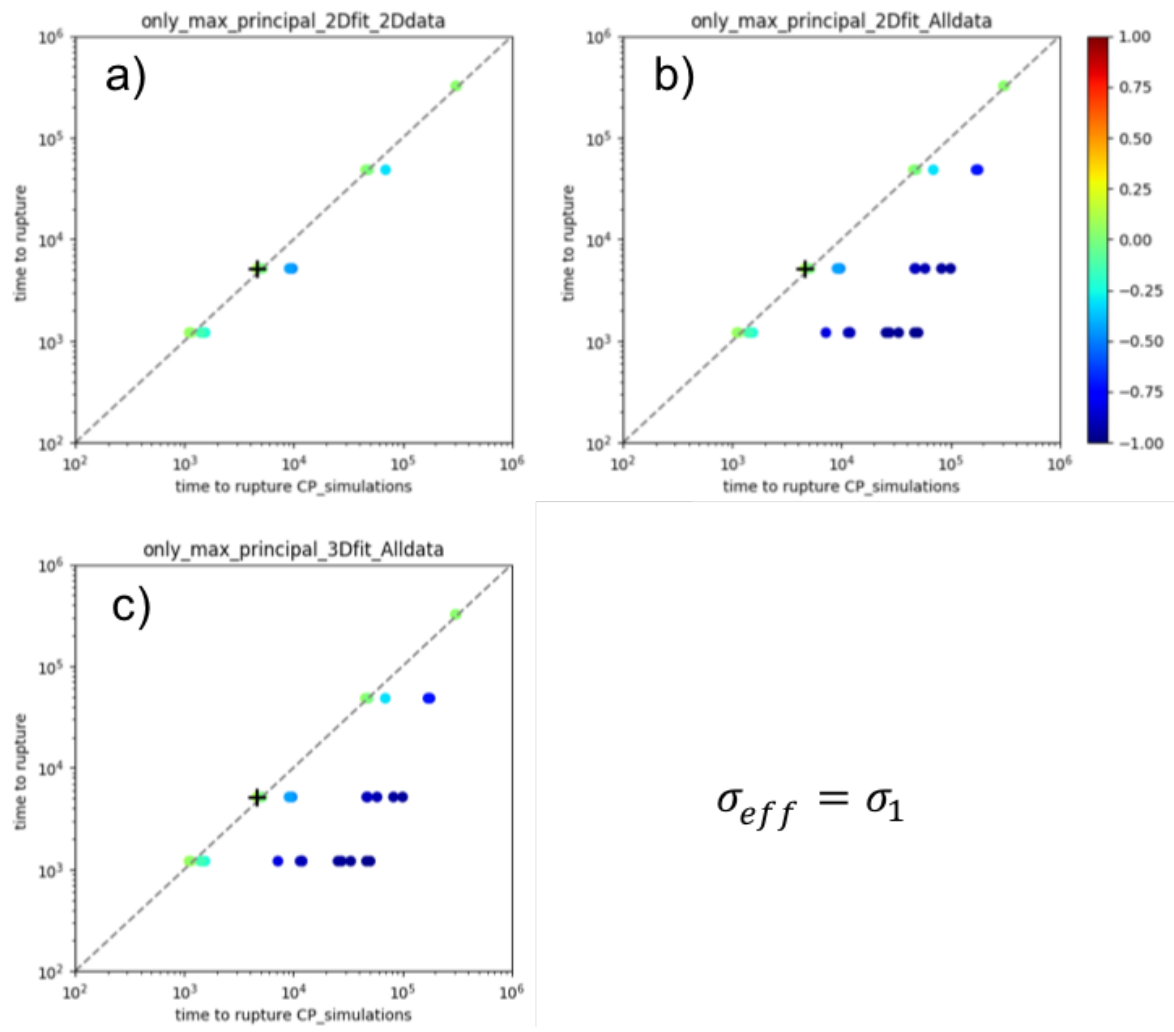

$$
\sigma_{e f f}=\sigma_{1}
$$

Figure 5.4: Comparison RSMRE distribution for the max stress of simulated versus predicted time to rupture. Color represent RSMRE error. a) biaxial fit versus biaxial data, b) biaxial fit versus all data c) triaxial fit versus triaxial data 
Initial study of notch sensitivity of Grade 91 using mechanisms motivated crystal plasticity finite element method
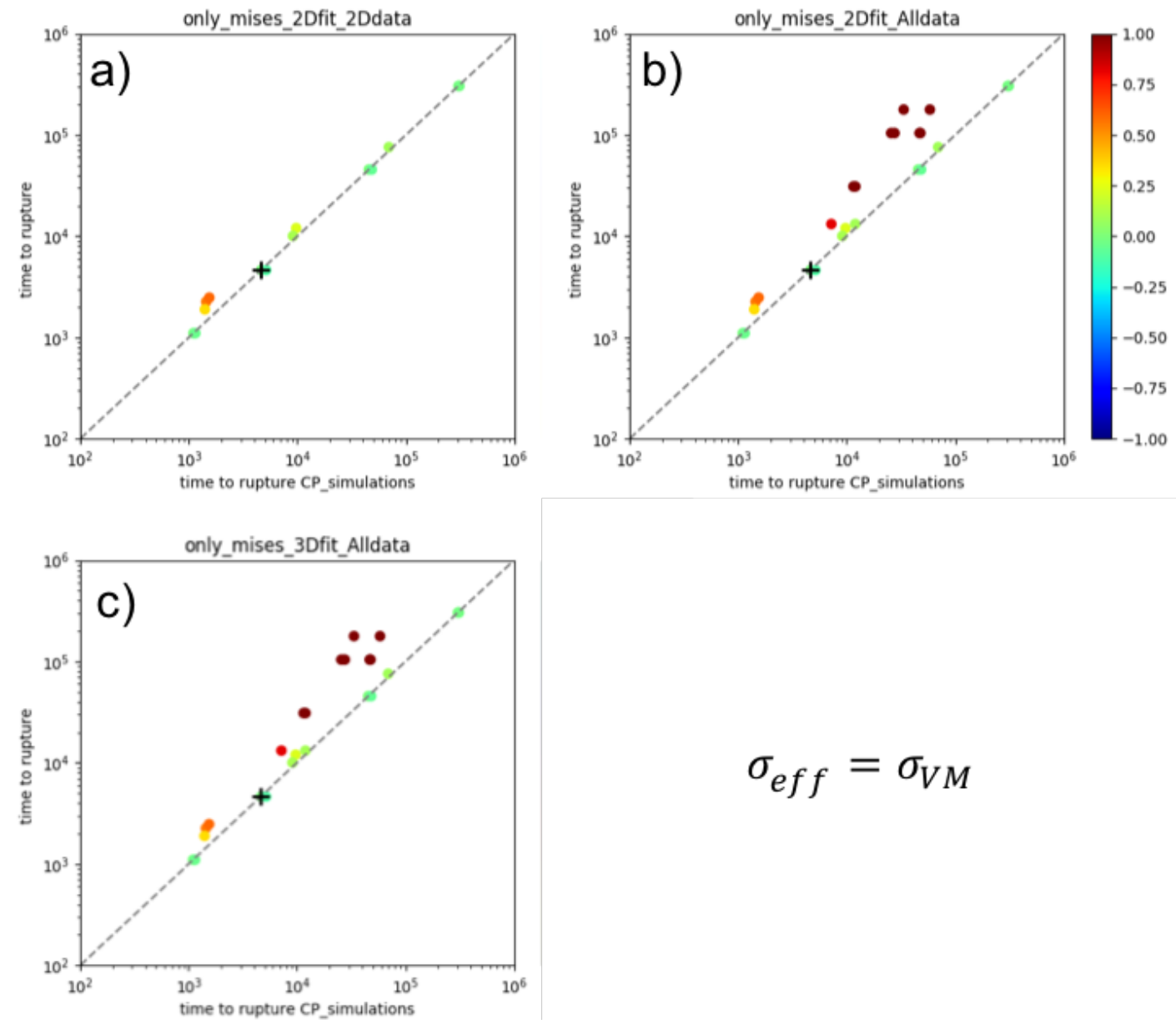

$$
\sigma_{e f f}=\sigma_{V M}
$$

Figure 5.5: Comparison RSMRE distribution for the mises stress of simulated versus predicted time to rupture. Color represent RSMRE error. a) biaxial fit versus biaxial data, b) biaxial fit versus all data c) triaxial fit versus triaxial data 
Initial study of notch sensitivity of Grade 91 using mechanisms motivated crystal plasticity finite element method
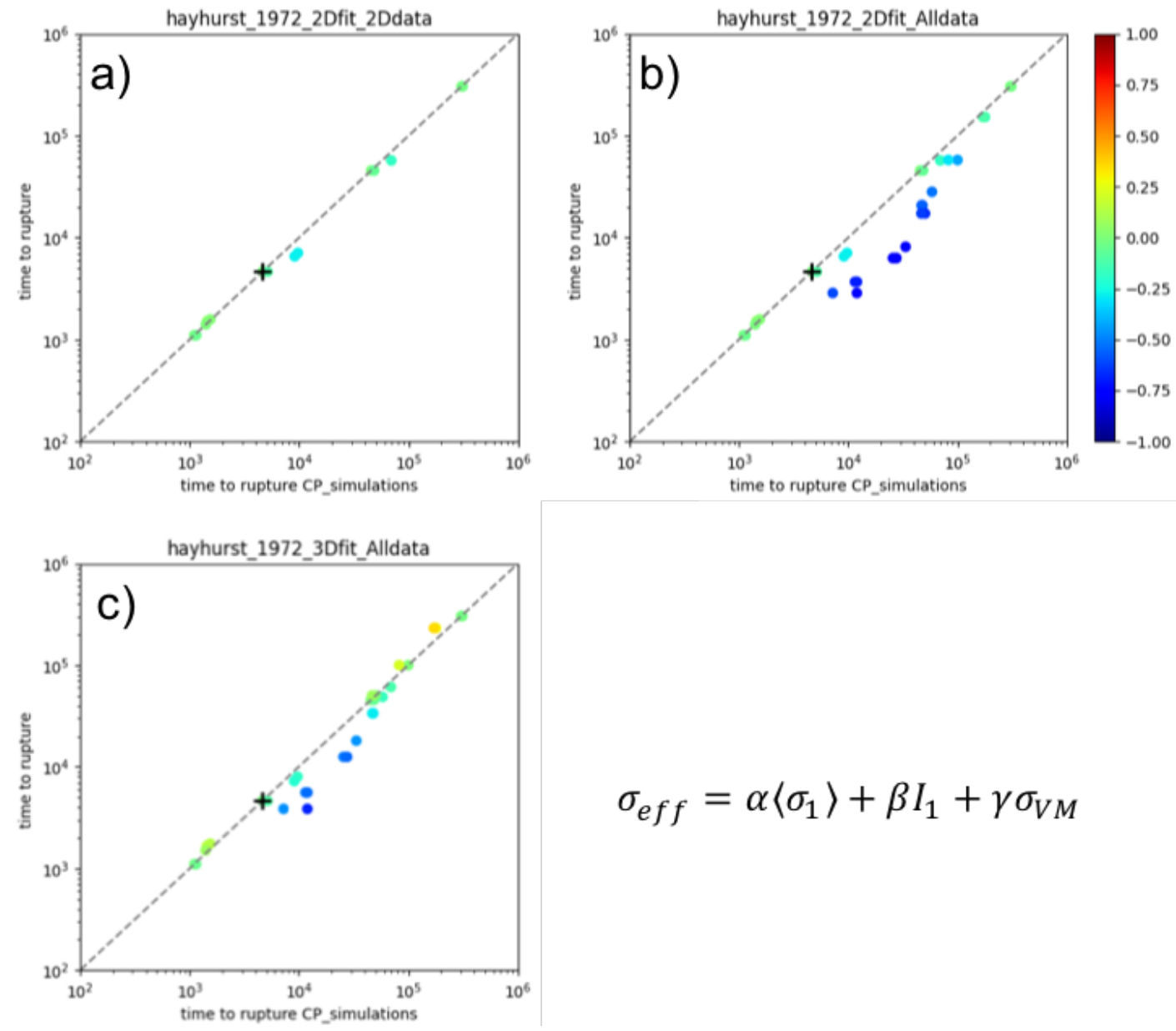

$$
\sigma_{e f f}=\alpha\left\langle\sigma_{1}\right\rangle+\beta I_{1}+\gamma \sigma_{V M}
$$

Figure 5.6: Comparison RSMRE distribution for the Hayhurst_1972 stress of simulated versus predicted time to rupture. Color represent RSMRE error. a) biaxial fit versus biaxial data, b) biaxial fit versus all data c) triaxial fit versus triaxial data 
Initial study of notch sensitivity of Grade 91 using mechanisms motivated crystal plasticity finite element method
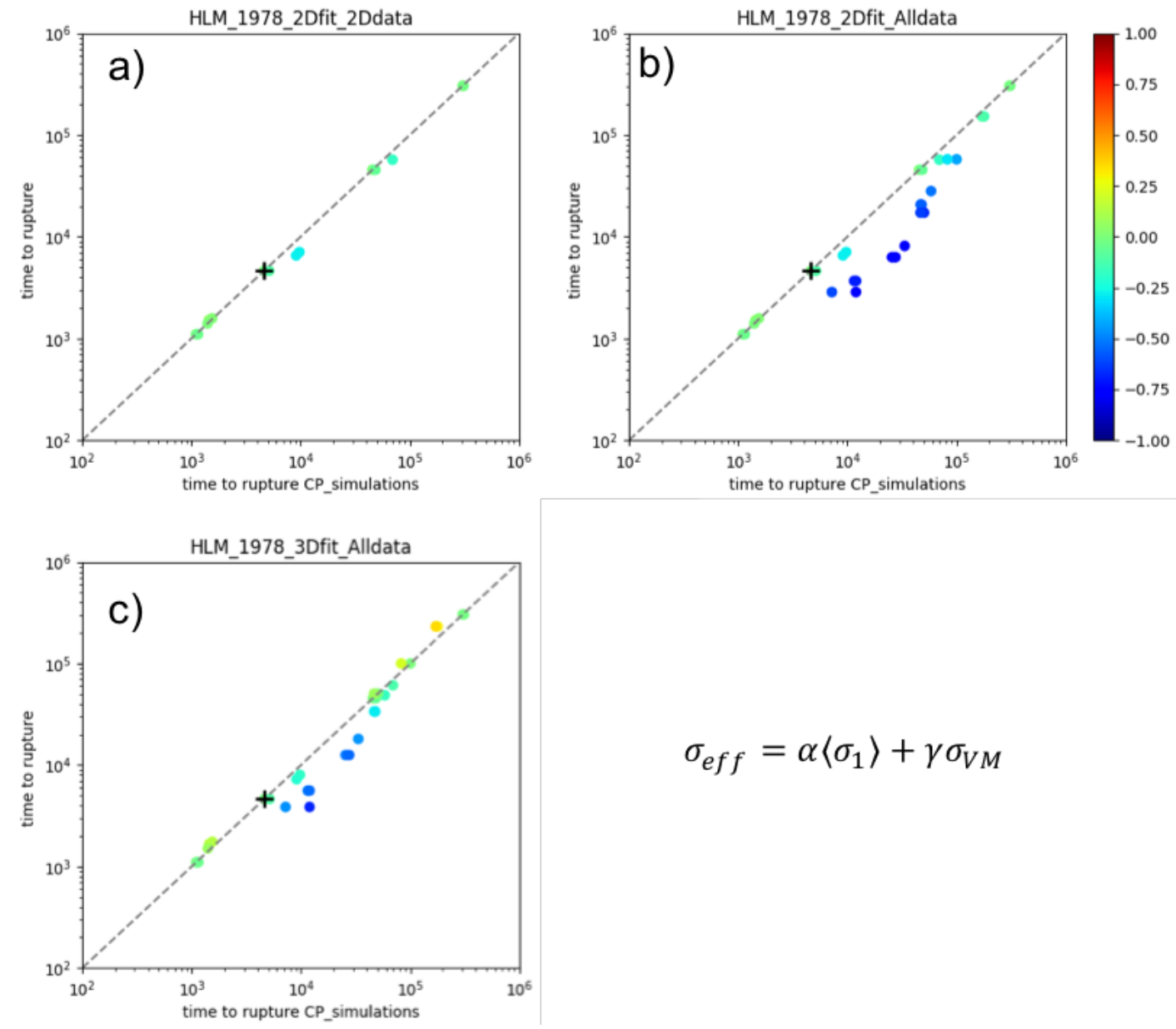

$$
\sigma_{e f f}=\alpha\left\langle\sigma_{1}\right\rangle+\gamma \sigma_{V M}
$$

Figure 5.7: Comparison RSMRE distribution for HLM_1978 stress of simulated versus predicted time to rupture. Color represent RSMRE error. a) biaxial fit versus biaxial data, b) biaxial fit versus all data c) triaxial fit versus triaxial data 
Initial study of notch sensitivity of Grade 91 using mechanisms motivated crystal plasticity finite element method
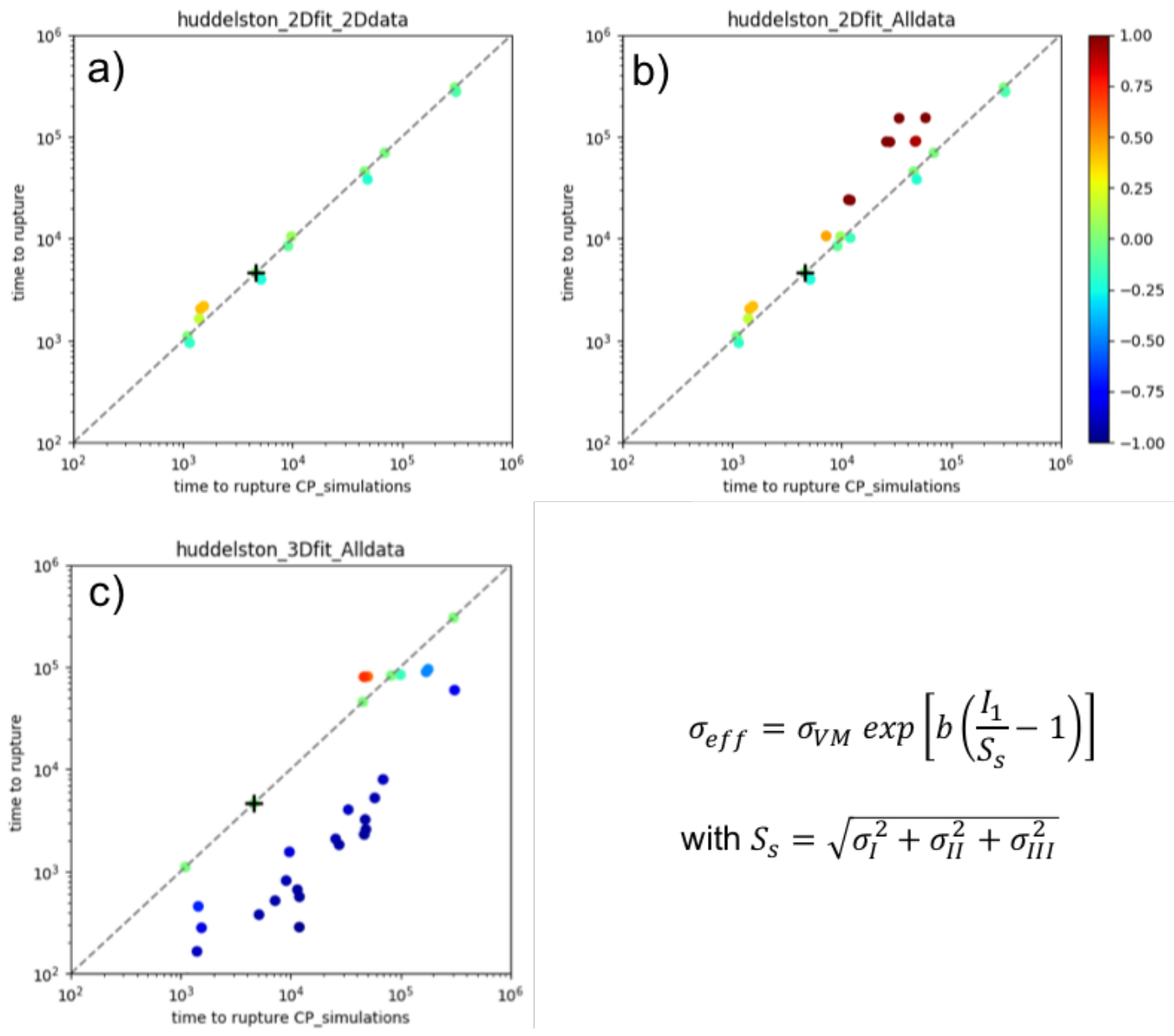

$$
\begin{gathered}
\sigma_{e f f}=\sigma_{V M} \exp \left[b\left(\frac{I_{1}}{S_{s}}-1\right)\right] \\
\text { with } S_{S}=\sqrt{\sigma_{I}^{2}+\sigma_{I I}^{2}+\sigma_{I I I}^{2}}
\end{gathered}
$$

Figure 5.8: Comparison RSMRE distribution for Huddleston stress of simulated versus predicted time to rupture. Color represent RSMRE error. a) biaxial fit versus biaxial data, b) biaxial fit versus all data c) triaxial fit versus triaxial data 
Initial study of notch sensitivity of Grade 91 using mechanisms motivated crystal plasticity finite element method
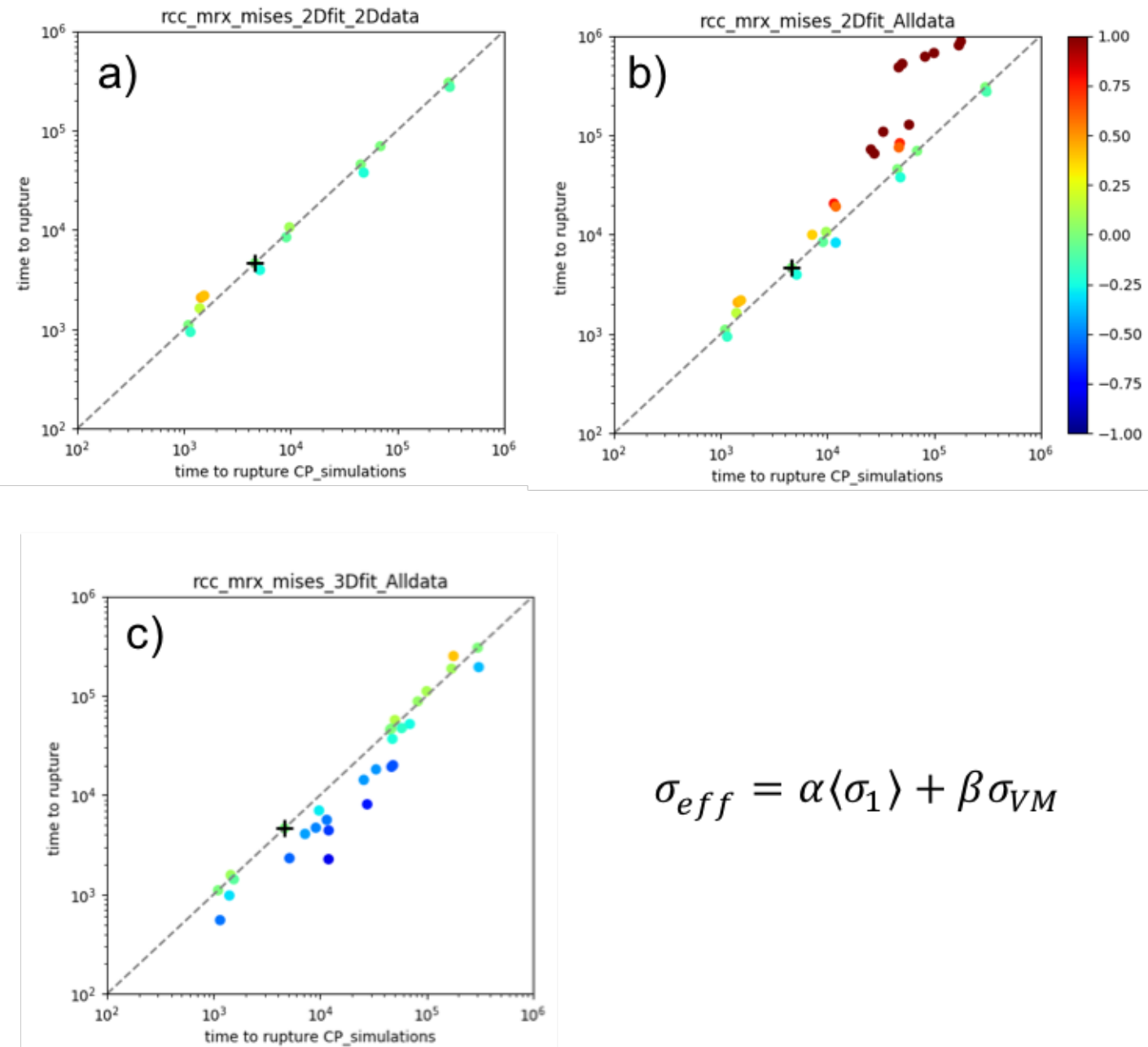

$$
\sigma_{e f f}=\alpha\left\langle\sigma_{1}\right\rangle+\beta \sigma_{V M}
$$

Figure 5.9: Comparison RSMRE distribution for the $R C C$-MRx_mises stress of simulated versus predicted time to rupture. Color represent RSMRE error. a) biaxial fit versus biaxial data, b) biaxial fit versus all data c) triaxial fit versus triaxial data 
Initial study of notch sensitivity of Grade 91 using mechanisms motivated crystal plasticity finite element method
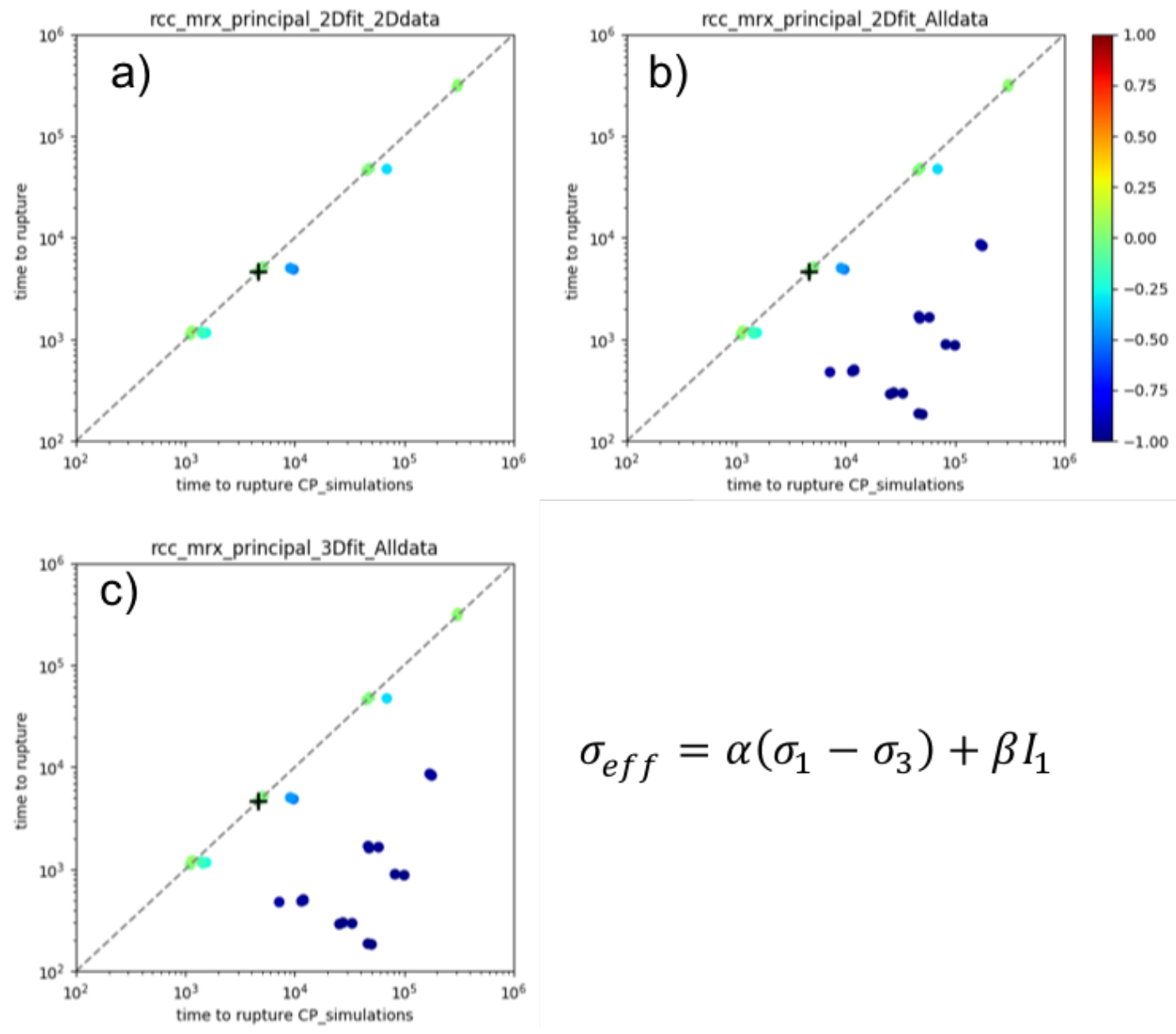

$$
\sigma_{e f f}=\alpha\left(\sigma_{1}-\sigma_{3}\right)+\beta I_{1}
$$

Figure 5.10: Comparison RSMRE distribution for the $R C C-M R x_{-}$max stress of simulated versus predicted time to rupture. Color represent RSMRE error. a) biaxial fit versus biaxial data, b) biaxial fit versus all data c) triaxial fit versus triaxial data 

Initial study of notch sensitivity of Grade 91 using mechanisms motivated crystal plasticity finite element method

September 2019

\section{Conclusions}

This report describes a physically-based CPFEM model for creep rupture in Grade 91 that can be used to predict the effect of stress triaxiality on the creep life of the material. The stress states in the core block of heat pipe microreactors are significantly different than those found in typical thin-walled pressure vessel components in conventional advanced reactor designs. As discussed in the report, the effective stresses used to correlate rupture times under multiaxial loads to uniaxial rupture data may not be accurate for core block designs because current effective stress measures have only been calibrated and tested against biaxial rupture data.

The CPFEM model can make predictions of creep rupture life for arbitrary stress states, including those found in reactor core blocks. Moreover, the physically based model developed here should be more accurate, compared to conventional empirical methods, when used to make predictions for loading conditions for which it was not directly calibrated. Therefore, the physically-based model should be an accurate method for assessing multiaxial rupture life.

However, despite its sophistication, the CPFEM simulations are still a model and therefore must be validated. Previous work has extensively validated the model against uniaxial creep rupture data. Arguably, because the physics remains the same for uniaxial, biaxial, or triaxial loading, this validation should be sufficient to make predictions of creep rupture in triaxial conditions. However, the model should also be validated against a limited number of multiaxial rupture tests. Ideally, these tests would be conducted under truly 3D triaxial stress states. However, to our knowledge no such triaxial creep test method exists. Validation against biaxial results could at least be used to demonstrate that the model is accurately extrapolating from uniaxial to multiaxial stress states. There are well-established methods for conducting biaxial rupture tests, discussed above. However, to our knowledge biaxial creep tests have not be done for Grade 91 in the past. Therefore, we recommend a limited experimental program aimed at generating a few biaxial rupture tests on Grade 91 at relevant heat pipe reactor temperatures in order to validate the CPFEM model and the effective stress recommendations made in this report.

If validated with multiaxial creep tests, the model results presented here suggest current design practice should be altered when designing Grade 91 high temperature components with significant stress triaxiality. Future work would be needed to implement these changes in relevant design codes and standards. The path forward would be to use the uniaxial rupture model developed from the CPFEM calculations in [37] in combination with the calibrated Hayhurst effective stress described in this report. The combination of this effective stress and uniaxial rupture model captures all the behavior observed in the physically based CPFEM model and not accounted for in current design codes: mechanism shifts between high and low stress uniaxial rupture life regimes, a transition between notch strengthening and weakening, and the correlation between multiaxial stress and rupture. 

Initial study of notch sensitivity of Grade 91 using mechanisms motivated crystal plasticity finite element method

September 2019

\section{Acknowledgements}

The research was sponsored by the U.S. Department of Energy (DOE), under Contract No. DE-AC02-06CH11357 with Argonne National Laboratory, managed and operated by UChicago Argonne LLC. Programmatic direction was provided by the Office of Nuclear Reactor Deployment of the Office of Nuclear Energy (NE). The authors gratefully acknowledge the support provided by Tom Sowinski, DOE-NE, Federal Manager, Microreactors Campaign, Advanced Reactor Technologies (ART) Program, Sue Lesica, DOE-NE, Federal Manager, Advanced Materials, ART Program, and Jess Gehin, Idaho National Laboratory, National Technical Director, Microreactors Campaign, ART Program. 

Initial study of notch sensitivity of Grade 91 using mechanisms motivated crystal plasticity finite element method

September 2019

\section{Bibliography}

[1] AFCEN. RCC-MRx: AFCEN, 2015.

[2] American Society of Mechanical Engineers. Section III, Division 5. In ASME Boiler and Pressure Vessel Code. 2019.

[3] Argonne National Laboratory. NEML: the Nuclear Engineering material Model Library. https://github.com/Argonne-National-Laboratory/neml, 2019.

[4] Robert J Asaro. Crystal plasticity. Journal of applied mechanics, 50(4b):921-934, 1983.

[5] Robert J Asaro and JR Rice. Strain localization in ductile single crystals. Journal of the Mechanics and Physics of Solids, 25(5):309-338, 1977.

[6] Satish Balay, Shrirang Abhyankar, Mark F. Adams, Jed Brown, Peter Brune, Kris Buschelman, Lisandro Dalcin, Alp Dener, Victor Eijkhout, William D. Gropp, Dmitry Karpeyev, Dinesh Kaushik, Matthew G. Knepley, Dave A. May, Lois Curfman McInnes, Richard Tran Mills, Todd Munson, Karl Rupp, Patrick Sanan, Barry F. Smith, Stefano Zampini, Hong Zhang, and Hong Zhang. PETSc users manual. Technical Report ANL95/11 - Revision 3.11, Argonne National Laboratory, 2019.

[7] Satish Balay, Shrirang Abhyankar, Mark F. Adams, Jed Brown, Peter Brune, Kris Buschelman, Lisandro Dalcin, Alp Dener, Victor Eijkhout, William D. Gropp, Dmitry Karpeyev, Dinesh Kaushik, Matthew G. Knepley, Dave A. May, Lois Curfman McInnes, Richard Tran Mills, Todd Munson, Karl Rupp, Patrick Sanan, Barry F. Smith, Stefano Zampini, Hong Zhang, and Hong Zhang. PETSc Web page. https://www.mcs.anl.gov/petsc, 2019.

[8] W. F. Brown, M. H. Jones, and D. P. Newman. Influence of Sharp Notches on the StressRupture Characteristics of Several Heat-Resisting Alloys. In Symposium on Strength and Ductility of Metals at Elevated Temperatures: With Particular Reference to Effects of Notches and Metallurgical Changes, pages 25-48, 1953.

[9] ACF Cocks and MF Ashby. On creep fracture by void growth. Progress in materials science, 27(3-4):189-244, 1982.

[10] John D Cook. Basic properties of the soft maximum. 2011.

[11] E. A. Davis and M. J. Manjone. Effect of Notch Geometry on Rupture Strength at Elevated Temperatures. In Symposium on Strength and Ductility of Metals at Elevated Temperatures: With Particular Reference to Effects of Notches and Metallurgical Changes, pages 67-92, 1953.

[12] BF Dyson. Continuous cavity nucleation and creep fracture. Scripta Metallurgica, 17 (1):31-38, 1983.

[13] BF Dyson and D McLean. Creep of nimonic 80a in torsion and tension. Metal science, 11(2):37-45, 1977. 
Initial study of notch sensitivity of Grade 91 using mechanisms motivated crystal plasticity finite element method

September 2019

[14] EDF Energy Nuclear Generation Ltd. R5: Assessment Procedure for the High Temperature Response of Structures. British Energy, 2012.

[15] D. E. Furman and A. M. Talbot. Notch rupture tests of Inconel X and Nimonic 80A. In Symposium on Strength and Ductility of Metals at Elevated Temperatures: With Particular Reference to Effects of Notches and Metallurgical Changes, pages 59-66, 1953.

[16] Derek R Gaston, Cody J Permann, John W Peterson, Andrew E Slaughter, David Andrš, Yaqi Wang, Michael P Short, Danielle M Perez, Michael R Tonks, Javier Ortensi, et al. Physics-based multiscale coupling for full core nuclear reactor simulation. Annals of Nuclear Energy, 84:45-54, 2015.

[17] RM Goldhoff. Uniaxial creep-rupture behavior of low-alloy steel under variable loading conditions. Journal of Basic Engineering, 87(2):374-378, 1965.

[18] Arthur L Gurson. Continuum theory of ductile rupture by void nucleation and growth. part i. yield criteria and flow rules for porous ductile media. Technical report, Brown Univ., Providence, RI (USA). Div. of Engineering, 1975.

[19] David Robert Hayhurst, PR Dimmer, and CJ Morrison. Development of continuum damage in the creep rupture of notched bars. Philosophical Transactions of the Royal Society of London. Series A, Mathematical and Physical Sciences, 311(1516):103-129, 1984.

[20] DR Hayhurst. Creep rupture under multi-axial states of stress. Journal of the Mechanics and Physics of Solids, 20(6):381-382, 1972.

[21] DR Hayhurst. A biaxial-tension creep-rupture testing machine. Journal of Strain Analysis, 8(2):119-123, 1973.

[22] DR Hayhurst and JT Henderson. Creep stress redistribution in notched bars. International Journal of Mechanical Sciences, 19(3):133-146, 1977.

[23] R Hill and JR Rice. Constitutive analysis of elastic-plastic crystals at arbitrary strain. Journal of the Mechanics and Physics of Solids, 20(6):401-413, 1972.

[24] R. Huddleston. Two-parameter failure model improves time- independent and timedependent failure predictions. Technical Report UCRL-TR-202300, Lawrence Livermore National Laboratory, 2003.

[25] RL Huddleston. An improved multiaxial creep-rupture strength criterion. Journal of Pressure Vessel Technology, 107(4):421-429, 1985.

[26] RL Huddleston. Assessment of an improved multiaxial strength theory based on creeprupture data for type 316 stainless steel. Journal of pressure vessel technology, 115(2): 177-184, 1993. 
Initial study of notch sensitivity of Grade 91 using mechanisms motivated crystal plasticity finite element method

September 2019

[27] K. Kimura, H. Kushima, and K. Sawada. Long-term creep deformation property of modified 9Cr-1Mo steel. Materials Science and Engineering A, 510-511(C):58-63, 2009.

[28] S. Kok, A. J. Beaudoin, and D. A. Tortorelli. A polycrystal plasticity model based on the mechanical threshold. International Journal of Plasticity, 18(5-6):715-741, oct 2002 .

[29] Kyle C Koppenhoefer, Arne S Gullerud, Claudio Ruggieri, Robert H Dodds Jr, and BE Healey. Warp3d: Dynamic nonlinear analysis of solids using a preconditioned conjugate gradient software architecture. Technical report, University of Illinois Engineering Experiment Station. College of , 1994.

[30] F. R. Larson and J. Miller. A time-temperature relationship for rupture and creep stresses. Transactions of the ASME, 74:765-771, 1952.

[31] FA Leckie and DR Hayhurst. Creep rupture of structures. Proceedings of the Royal Society of London. A. Mathematical and Physical Sciences, 340(1622):323-347, 1974.

[32] L. C. Lim. Cavity nucleation at high temperatures involving pile-ups of grain boundary dislocations. Acta Metallurgica, 35(7):1663-1673, 1987.

[33] Patrick McClure, David Poston, D. V. Rao, and Robert Reid. Design of megawatt power level heat pipe reactors. Technical Report LANL-UR-15-28840, Los Alamos National Laboratory, 2015.

[34] M. C. Messner, T. J. Truster, K. B. Cochran, D. M. Parks, and T.-L. Sham. FY17 status report on the micromechanical finite element modeling of creep fracture of Grade 91 steel. Technical Report ANL-ART-95, Argonne National Laboratory, 2017.

[35] Mark Messner, Armand Beaudoin, and Robert Dodds. Consistent crystal plasticity kinematics and linearization for the implicit finite element method. Engineering Computations, 32(6):1526-1548, 2015.

[36] Mark Christian Messner, Omar Nassif, Ran Ma, Timothy Truster, Kristine Cochran, David Parks, and T-L Sham. Combined crystal plasticity and grain boundary modeling of creep in ferritic-martensitic steels, part 2: The effect of stress and temperature on engineering and microstructural properties. Modelling and Simulation in Materials Science and Engineering, 2019.

[37] MC Messner, A Rovinelli, DM Parks, and T-L Sham. Evaluation of statistical variation of microstructural properties and temperature effects on creep fracture of grade 91 . Technical report, Argonne National Lab.(ANL), Argonne, IL (United States), 2018.

[38] Omar Nassif, Timothy Truster, Ran Ma, Kristine Cochran, David Parks, Mark Christian Messner, and T-L Sham. Combined crystal plasticity and grain boundary modeling of creep in ferritic-martensitic steels, part 1: Theory and implementation. Modelling and Simulation in Materials Science and Engineering, 2019. 
Initial study of notch sensitivity of Grade 91 using mechanisms motivated crystal plasticity finite element method

September 2019

[39] A Needleman. Void growth in an elastic-plastic medium. Journal of Applied Mechanics, 39(4):964-970, 1972.

[40] A. Needleman and J. R. Rice. Plastic Creep Flow Effects in the Diffusive Cavitation of Grain Boundaries. Acta Metallurgica, 28(10):1315-1332, 1980.

[41] Romain Quey, PR Dawson, and Fabrice Barbe. Large-scale 3d random polycrystals for the finite element method: Generation, meshing and remeshing. Computer Methods in Applied Mechanics and Engineering, 200(17-20):1729-1745, 2011.

[42] J R_ Rice and Dennis Michael Tracey. On the ductile enlargement of voids in triaxial stress fields. Journal of the Mechanics and Physics of Solids, 17(3):201-217, 1969.

[43] James R Rice. Inelastic constitutive relations for solids: an internal-variable theory and its application to metal plasticity. Journal of the Mechanics and Physics of Solids, 19 (6):433-455, 1971.

[44] Franz Roters, Philip Eisenlohr, Luc Hantcherli, Denny Dharmawan Tjahjanto, Thomas R Bieler, and Dierk Raabe. Overview of constitutive laws, kinematics, homogenization and multiscale methods in crystal plasticity finite-element modeling: Theory, experiments, applications. Acta Materialia, 58(4):1152-1211, 2010.

[45] G. Sachs and W. F. Brown. A survey of embrittlement and notch sensitivity of heat resisting steels. In Symposium on Strength and Ductility of Metals at Elevated Temperatures: With Particular Reference to Effects of Notches and Metallurgical Changes, pages 6-24, 1953.

[46] T. L. Sham and A. Needleman. Effects of triaxial stressing on creep cavitation of grain boundaries. Acta Metallurgica, 31(6):919-926, 1983.

[47] MW Spindler. The multiaxial creep ductility of austenitic stainless steels. Fatigue $\mathbb{E}$ fracture of engineering materials \& structures, 27(4):273-281, 2004.

[48] SE Stanzl, AS Argon, and EK Tschegg. Diffusive intergranular cavity growth in creep in tension and torsion. Acta metallurgica, 31(6):833-843, 1983.

[49] J. W. Sterbentz, J. E. Werner, M. G. McKellar, A. J. Hummel, J. C. Kennedy, R. N. Wright, and J. M. Biersdorf. Special purpose nuclear reactor $(5 \mathrm{mv})$ for reliable power at remote sites assessment report. Technical Report INL/EXT-16-40741, Idaho National Laboratory, 2017.

[50] Viggo Tvergaard and Alan Needleman. Analysis of the cup-cone fracture in a round tensile bar. Acta metallurgica, 32(1):157-169, 1984.

[51] Erik Van der Giessen, M W D der Burg, A Needleman, and V Tvergaard. Void growth due to creep and grain boundary diffusion at high triaxialities. Journal of the Mechanics and Physics of Solids, 43(1):123-165, 1995. 
Initial study of notch sensitivity of Grade 91 using mechanisms motivated crystal plasticity finite element method

[52] J. K Wright and R. N. Wright. Creep rupture of pressurized alloy 617 tubes. Technical Report INL/EXT-13-30251, Idaho National Laboratory, 2013.

[53] R Wu and R Sandstrom. Strain dependence of creep cavity nucleation in low alloy and 12\% Cr steels. Materials Science and Technology, 12(5):405-415, 1996.

[54] Hua-Tang Yao, Fu-Zhen Xuan, Zhengdong Wang, and Shan-Tung Tu. A review of creep analysis and design under multi-axial stress states. Nuclear Engineering and Design, 237(18):1969-1986, 2007. 

Initial study of notch sensitivity of Grade 91 using mechanisms motivated crystal plasticity finite element method

September 2019

\section{A Stress analysis of a core-block concept}

The following is a preliminary version of a report describing an inelastic analysis of a core block structure completed at Argonne National Laboratory. The triaxiality results were abstracted in Chapter 2 above. 


\section{Objectives}

Special purpose reactor was designed to supply energy on remote areas or military bases, where electrical power is needed and it is difficult to supply conventional fuels (coal, LNG, diesel, etc.) through transportation regularly. The presented study focuses on the reactor thermal-structural analysis, and with limited information on service loads, tries to apply ASME BPVC Section III Div.5 to estimate possible failure modes of the current design, especially those related to creep damage.

\section{Scope}

The scope of this analysis was limited to the central part of the special purpose reactor, the core block, which holds fuel pins in place and work partially as heat pipe to take the reactor heat away for electric power generation. The working conditions were assumed to be between $677^{\circ} \mathrm{C}$ and $700{ }^{\circ} \mathrm{C}$, with possible cooling down and heating up between those two temperatures. The main load will be thermal load due to inhomogeneity of the heat flux of fuel pin nuclear reactions; pressure load was assumed to be small; other loads, such as seismic load were not considered in this report. Residual stresses, due to core block manufacturing and previous cycles, were assumed to be less than the material yield strength at $677^{\circ} \mathrm{C}$.

\section{Methodology}

A finite element model was setup with the core block geometry, including the holes for fuel pins and heat pipes. Material mechanical properties of the model were from ASME BPVC Section III, Div.5 [1]. An initial analysis based on secondary stress range were accomplished by following the procedures of [1], both elastic and inelastic analysis were included and for future creep damage calculations, ductility exhaustion, even though not included in [1], was recommended to reduce creep damage calculation result conservatism introduced by following time-fraction method in [1].

\section{Overview of Analysis}

The core block is the central part of the special purpose reactor, which generates electricity for remote sites or military bases where the conventional fuels is not easily accessible. The core block contains holes for fuel pins, which generate heat due to nuclear reactions, and holes for heat pipe, which take the generated heat away for power generation. The magnitude of the heat flux from the fuel pins shows a distribution along their axial direction, and the resulted inhomogeneous temperature field in the core block leads to high thermal stresses. The webs between the holes prone to fail as they can be as thin as $1 \mathrm{~mm}$ or less, and under high stress at high temperature ( 700 ${ }^{0} \mathrm{C}$ ), they are the foci of the presented thermal-mechanical analysis and also part of the reasons the core block was chosen for this study presented in the report.

The simulation was run workstation with operating system Windows 10 Pro, 2 Intel ${ }^{\circledR}$ Xeon ${ }^{\circledR}$ E52687W CPUs with 24 cores and 500GB memory. The FEA software is ANSYS Mechanical version 19.2. 
Initial study of notch sensitivity of Grade 91 using mechanisms motivated crystal plasticity finite element method

September 2019

\section{Assumptions}

The following assumptions were made for FEA modeling:

1. Heat flux on the fuel pin hole walls is a function of the axial location only.

2. Between the fuel pin outside wall and the fuel pin hole wall, heat transfer only occurs in the radial direction, i.e., no heat transfer along the fuel pin axial direction.

3. Material is isotropic and homogeneous, possible thermal aging, irradiation embrittlement/swelling/creep were neglected.

4. Residual stresses are not included in the model, but during the analysis, the residual stress magnitude was assumed to be equal or less than the material yield strength.

\section{Geometry and Mesh}

\subsection{Geometry}

The geometry of the core block FEA model is displayed in Figure 6.1 blow, there are totally 352 fuel rod holes (dia. 14.25mm ones) and 204 heat pipe holes (dia. 15.75mm ones) in the model.

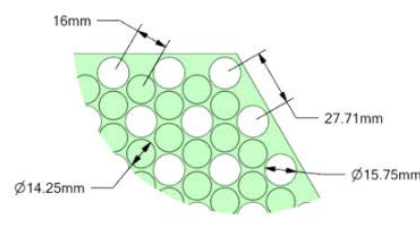

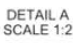

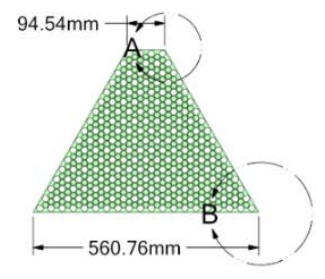

SCALE 2:20

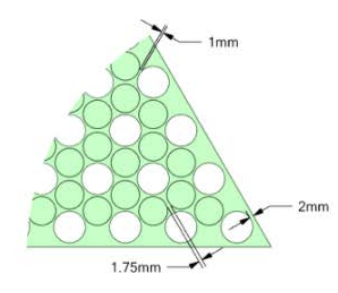

DETALB
SCAL 1.2

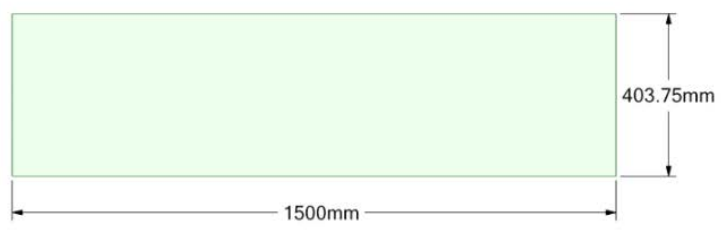

Figure 6.1 Core blocks with fuel rods (for a better view of holes).

\subsection{Mesh}

The FEA model mesh contains about one million quadratic solid elements (SOLID186), with the element size on the two end planes around $0.8 \mathrm{~mm}$ and 20 elements along the core block height. Mesh refinement study verified the FEA results were mesh-independent. 
Initial study of notch sensitivity of Grade 91 using mechanisms motivated crystal plasticity finite element method September 2019

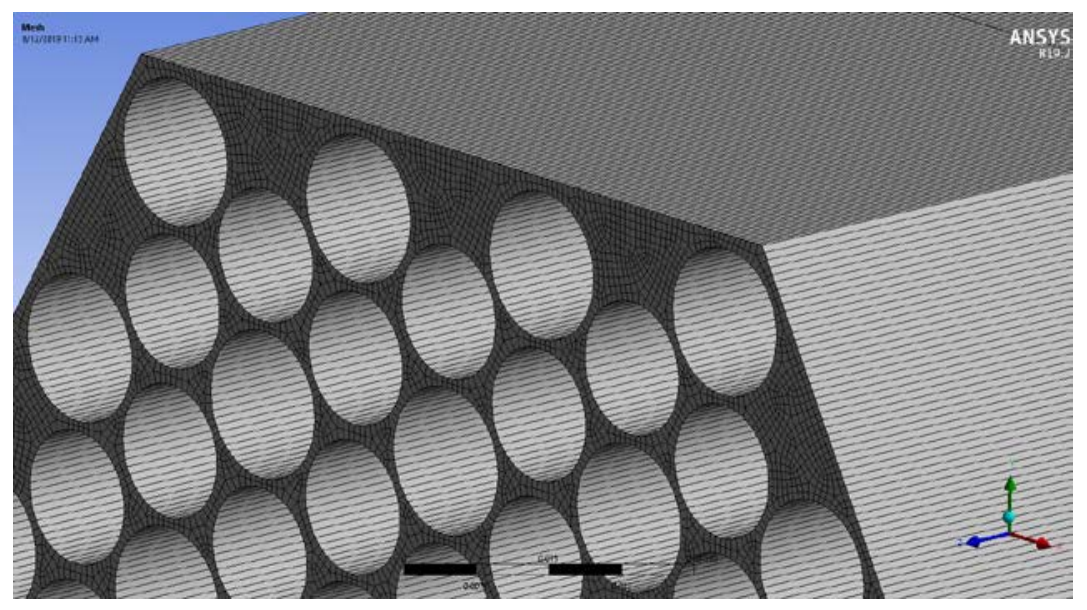

Figure 6.2 FEA model mesh.

\section{Materials}

The core block is designed to be made of Type 316 stainless steel, the material property data were provided by [1], which gave a density of $8030 \mathrm{~kg} / \mathrm{m}^{3}$ and a Poisson's ratio of 0.31 . Other material property data from [1] are shown in Table 7.1 to Table 7.5 below. As the whole part is designed to work around $700{ }^{0} \mathrm{C}$, material creep is inevitable, so Table 7.5 contains material creep law information.

\begin{tabular}{|c|c|}
\hline Temperature (degC) & Young's Modulues (Pa) \\
\hline 25 & $1.95 \mathrm{E}+11$ \\
\hline 100 & $1.89 \mathrm{E}+11$ \\
\hline 150 & $1.86 \mathrm{E}+11$ \\
\hline 200 & $1.83 \mathrm{E}+11$ \\
\hline 250 & $1.79 \mathrm{E}+11$ \\
\hline 300 & $1.76 \mathrm{E}+11$ \\
\hline 350 & $1.72 \mathrm{E}+11$ \\
\hline 400 & $1.69 \mathrm{E}+11$ \\
\hline 450 & $1.65 \mathrm{E}+11$ \\
\hline 500 & $1.60 \mathrm{E}+11$ \\
\hline 550 & $1.56 \mathrm{E}+11$ \\
\hline 600 & $1.51 \mathrm{E}+11$ \\
\hline 650 & $1.46 \mathrm{E}+11$ \\
\hline 700 & $1.40 \mathrm{E}+11$ \\
\hline
\end{tabular}

Table 7.1 SS316 Young's modulus 
Initial study of notch sensitivity of Grade 91 using mechanisms motivated crystal plasticity finite element method September 2019

\begin{tabular}{|c|c|}
\hline Temperature (degC) & CTE $(\mathrm{mm} / \mathrm{mm} / \mathrm{degC})$ \\
\hline 20 & $1.53 \mathrm{E}-05$ \\
\hline 50 & $1.60 \mathrm{E}-05$ \\
\hline 75 & 1.65E-05 \\
\hline 100 & $1.70 \mathrm{E}-05$ \\
\hline 125 & $1.74 \mathrm{E}-05$ \\
\hline 150 & $1.78 \mathrm{E}-05$ \\
\hline 175 & $1.81 \mathrm{E}-05$ \\
\hline 200 & $1.84 \mathrm{E}-05$ \\
\hline 225 & $1.86 \mathrm{E}-05$ \\
\hline 250 & $1.88 \mathrm{E}-05$ \\
\hline 275 & $1.89 \mathrm{E}-05$ \\
\hline 300 & $1.91 \mathrm{E}-05$ \\
\hline 325 & 1.92E-05 \\
\hline 350 & $1.93 \mathrm{E}-05$ \\
\hline 375 & $1.94 \mathrm{E}-05$ \\
\hline 400 & $1.95 \mathrm{E}-05$ \\
\hline 425 & $1.96 \mathrm{E}-05$ \\
\hline 450 & $1.98 \mathrm{E}-05$ \\
\hline 475 & $2.00 \mathrm{E}-05$ \\
\hline 500 & $2.02 \mathrm{E}-05$ \\
\hline 525 & $2.04 \mathrm{E}-05$ \\
\hline 550 & $2.06 \mathrm{E}-05$ \\
\hline 575 & 2.09E-05 \\
\hline 600 & $2.11 \mathrm{E}-05$ \\
\hline 625 & $2.14 \mathrm{E}-05$ \\
\hline 650 & $2.16 \mathrm{E}-05$ \\
\hline 675 & 2.17E-05 \\
\hline 700 & 2.17E-05 \\
\hline 725 & $2.15 \mathrm{E}-05$ \\
\hline 750 & $2.12 \mathrm{E}-05$ \\
\hline 775 & $2.06 \mathrm{E}-05$ \\
\hline 800 & 1.97E-05 \\
\hline 825 & $1.84 \mathrm{E}-05$ \\
\hline
\end{tabular}

Table 7.2 SS316 thermal expansion coefficient. 
Initial study of notch sensitivity of Grade 91 using mechanisms motivated crystal plasticity finite element method September 2019

\begin{tabular}{|c|c|}
\hline Temperature (degC) & Thermal Conductivity (W/m2.c) \\
\hline 20 & 14.1 \\
\hline 50 & 14.6 \\
\hline 75 & 15 \\
\hline 100 & 15.4 \\
\hline 125 & 15.7 \\
\hline 150 & 16.1 \\
\hline 175 & 16.5 \\
\hline 200 & 16.8 \\
\hline 225 & 17.2 \\
\hline 250 & 17.6 \\
\hline 275 & 17.9 \\
\hline 300 & 18.3 \\
\hline 325 & 18.7 \\
\hline 350 & 19 \\
\hline 375 & 19.4 \\
\hline 400 & 19.7 \\
\hline 425 & 20.1 \\
\hline 450 & 20.5 \\
\hline 475 & 20.8 \\
\hline 500 & 21.2 \\
\hline 525 & 21.5 \\
\hline 550 & 21.9 \\
\hline 575 & 22.2 \\
\hline 600 & 22.6 \\
\hline 625 & 22.9 \\
\hline 650 & 23.2 \\
\hline 675 & 23.6 \\
\hline 700 & 23.9 \\
\hline 725 & 24.2 \\
\hline 750 & 24.6 \\
\hline
\end{tabular}

Table 7.3 SS316 thermal conductivity. 
Initial study of notch sensitivity of Grade 91 using mechanisms motivated crystal plasticity finite element method

September 2019

\begin{tabular}{|c|c|}
\hline Temperature (degC) & Specific Heat(J/(kg.C) \\
\hline 20 & 491.9 \\
\hline 50 & 499.5 \\
\hline 75 & 506.2 \\
\hline 100 & 511.4 \\
\hline 125 & 514.5 \\
\hline 150 & 519.4 \\
\hline 175 & 524.2 \\
\hline 200 & 525.7 \\
\hline 225 & 528.9 \\
\hline 250 & 533.3 \\
\hline 275 & 535.9 \\
\hline 300 & 540.0 \\
\hline 325 & 544.1 \\
\hline 350 & 546.4 \\
\hline 375 & 550.3 \\
\hline 400 & 552.5 \\
\hline 425 & 556.2 \\
\hline 450 & 561.1 \\
\hline 475 & 561.9 \\
\hline 500 & 566.5 \\
\hline 525 & 567.3 \\
\hline 550 & 570.6 \\
\hline 575 & 571.2 \\
\hline 600 & 574.4 \\
\hline 625 & 576.1 \\
\hline 650 & 576.7 \\
\hline 675 & 579.7 \\
\hline 700 & 581.3 \\
\hline 725 & 584.0 \\
\hline 750 & 590.1 \\
\hline
\end{tabular}

Table 7.4 SS316 Specific heat.

\begin{tabular}{|c|l|}
\hline Creep law: & $\mathcal{E}_{\mathrm{cr}}{ }^{\prime}=C_{1} * \sigma^{C_{2}} * e^{-C_{3} / T}$ \\
\hline$\sigma$ & Stress in MPa \\
\hline$T$ & Temperature in Kelvin \\
\hline$C_{1}$ & $2.25 \mathrm{e} 22 \% / \mathrm{hr}$ \\
\hline$C_{2}$ & 5 \\
\hline$C_{3}$ & $77280 \mathrm{~K}$ \\
\hline
\end{tabular}

Table 7.5 Creep law parameters for SS316.

\section{Loads and Boundary Conditions}

In the FEA model, only thermal load from the fuel rod nuclear reactions was considered, [2] provides the normalized peaking factor along axial locations of the fuel pins (see Figure 8.1). It was assumed the heat transfer between the fuel pin and the fuel pin hole wall was only along their radial direction, then the heat flux values at the fuel rod hole wall along the axil direction were 
Initial study of notch sensitivity of Grade 91 using mechanisms motivated crystal plasticity finite element method

September 2019

calculated and are shown on Table 8.1, Figure 8.2 shows the heat flux contour plot at fuel pin walls in the core block.

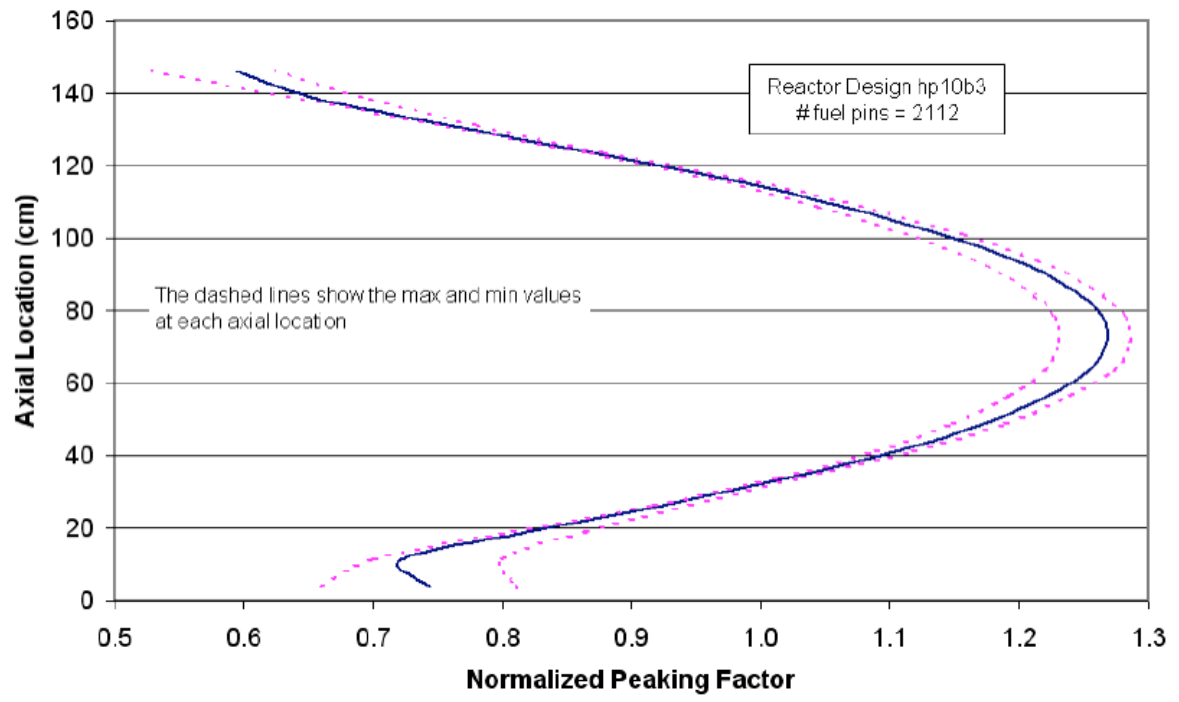

Figure 8.1 Axial normalized peaking factor distribution of power.

\begin{tabular}{|c|c|c|c|c|}
\hline $\begin{array}{c}\text { Position } \\
(\mathrm{cm})\end{array}$ & $\begin{array}{c}\text { Power/unit } \\
\text { length }(\mathrm{KW} / \mathrm{m})\end{array}$ & $\begin{array}{c}\text { Fuel hole } \\
\text { diameter }(\mathrm{mm})\end{array}$ & $\begin{array}{c}\text { Area for } 1 \mathrm{~m} \\
\text { length }\left(\mathrm{m}^{\wedge} 2\right)\end{array}$ & $\begin{array}{c}\text { Heat flux on } \\
\text { the fuel hole } \\
\text { wall }(\mathrm{w} / \mathrm{m} 2)\end{array}$ \\
\hline 0 & 1.15 & 14.25 & 0.04 & 25632.32 \\
\hline 10 & 1.09 & 14.25 & 0.04 & 24436.15 \\
\hline 20 & 1.29 & 14.25 & 0.04 & 28708.20 \\
\hline 40 & 1.67 & 14.25 & 0.04 & 37252.31 \\
\hline 60 & 1.90 & 14.25 & 0.04 & 42378.77 \\
\hline 80 & 1.94 & 14.25 & 0.04 & 43267.36 \\
\hline 100 & 1.77 & 14.25 & 0.04 & 39473.78 \\
\hline 120 & 1.42 & 14.25 & 0.04 & 31613.20 \\
\hline 140 & 0.99 & 14.25 & 0.04 & 22043.80 \\
\hline 150 & 0.87 & 14.25 & 0.04 & 19480.57 \\
\hline
\end{tabular}

Table 8.1 Heat flux at the fuel rod holes along the axial direction. 
Initial study of notch sensitivity of Grade 91 using mechanisms motivated crystal plasticity finite element method

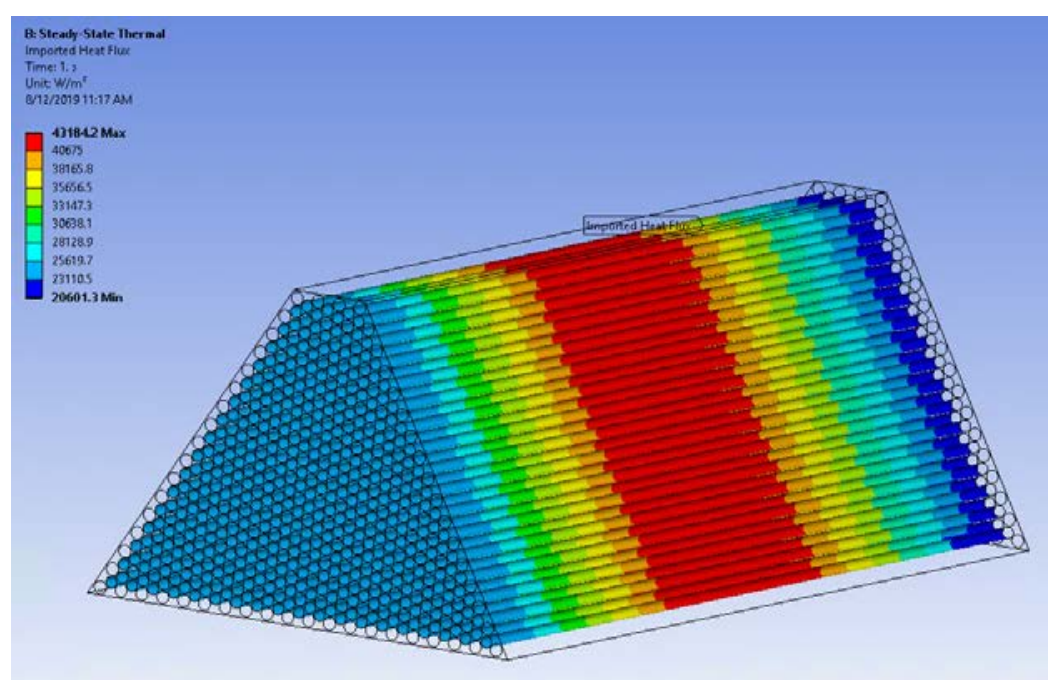

Figure 8.2 Heat flux on the fuel pin hole walls.

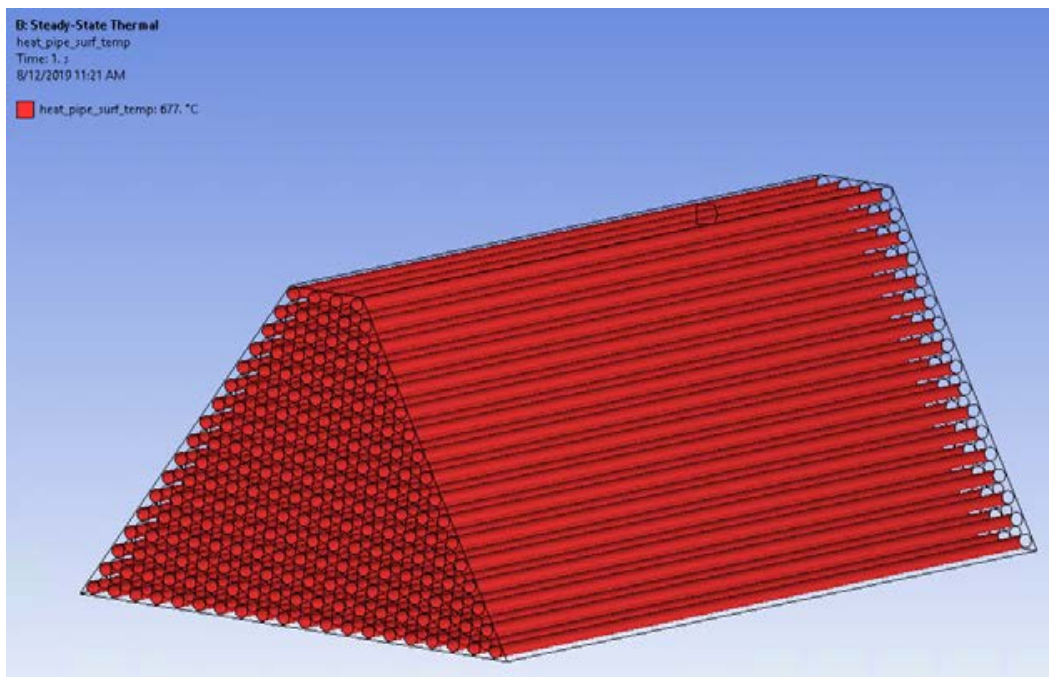

Figure 8.3 Constant temperature on the heat pipe hole walls.

For thermal boundary conditions, the heat pipe hole walls are at $677^{\circ} \mathrm{C}$, all other surfaces are thermally insulated, except those fuel pin hole walls with heat flux.

Mechanical boundary conditions are shown in Figure 8.4, the bottom plane displacement is fixed in $\mathrm{Z}$ direction, the top plane was constrained in a way it moves as a plane in $\mathrm{Z}$ direction, and two side planes are fixed in their planes. 
Initial study of notch sensitivity of Grade 91 using mechanisms motivated crystal plasticity finite element method

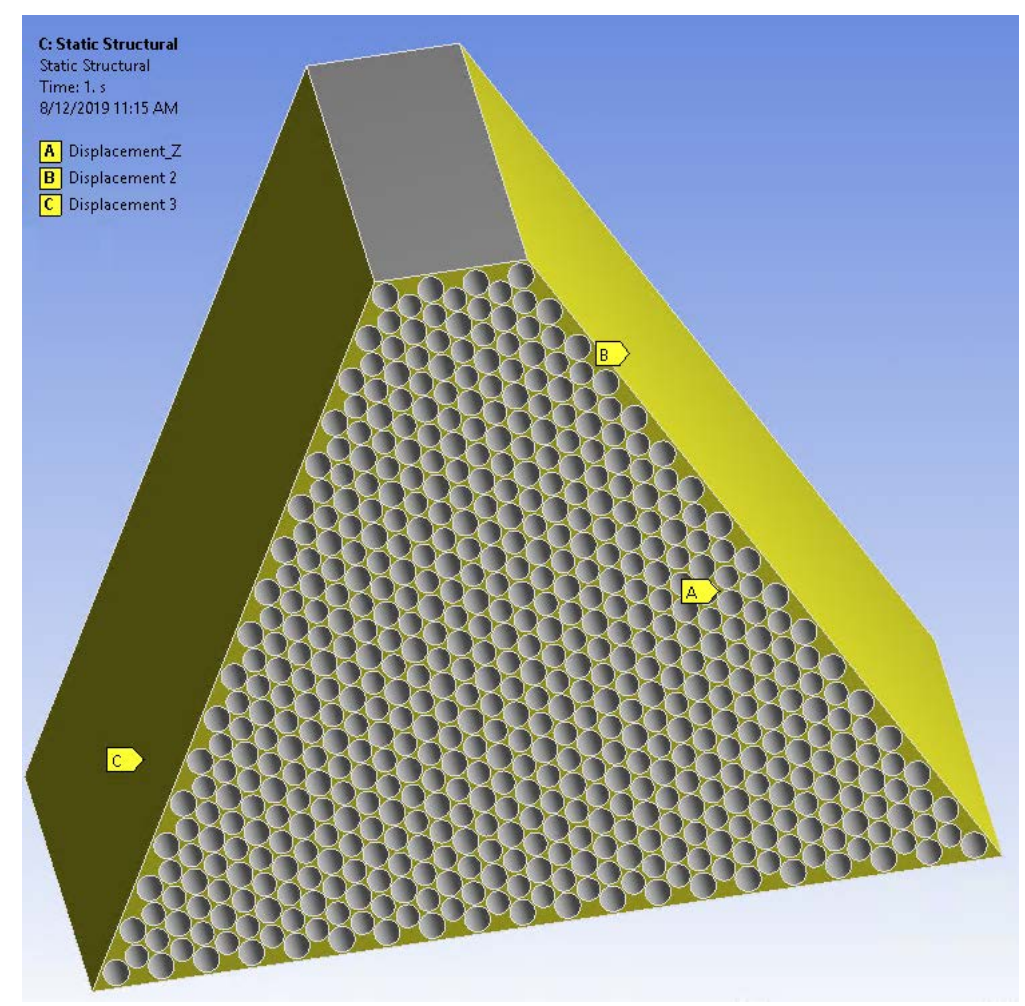

Figure 8.4 Mechanical boundary conditions

\section{Results and Analysis}

The FEA model with the boundary conditions and loads described above was run for the time period of 100,000 hours. During the first second, the thermal load, i.e., the heat flux at the fuel pin hole walls, was applied as a ramp to a full load without material creep, after that the temperature field keeps constants and the material creep was included for the rest of the simulation period.

\subsection{Thermal analysis results}

As the thermal load is applied as a ramp within the first second and keep constant for the rest of the simulation, only the temperature field at the end of first second is analyzed.

Figure 9.1 shows the temperature field at the mid-plane of the core block. As the heat pipe hole walls are kept at $677^{0} \mathrm{C}$, the regions around the heat pipe walls and regions around the core block edges are with that temperature. When the fuel pin walls are heated up by the fuel pins' nuclear reaction, there are temperature "hotter" zones at the webs between the fuel pin holes. 
Initial study of notch sensitivity of Grade 91 using mechanisms motivated crystal plasticity finite element method

September 2019

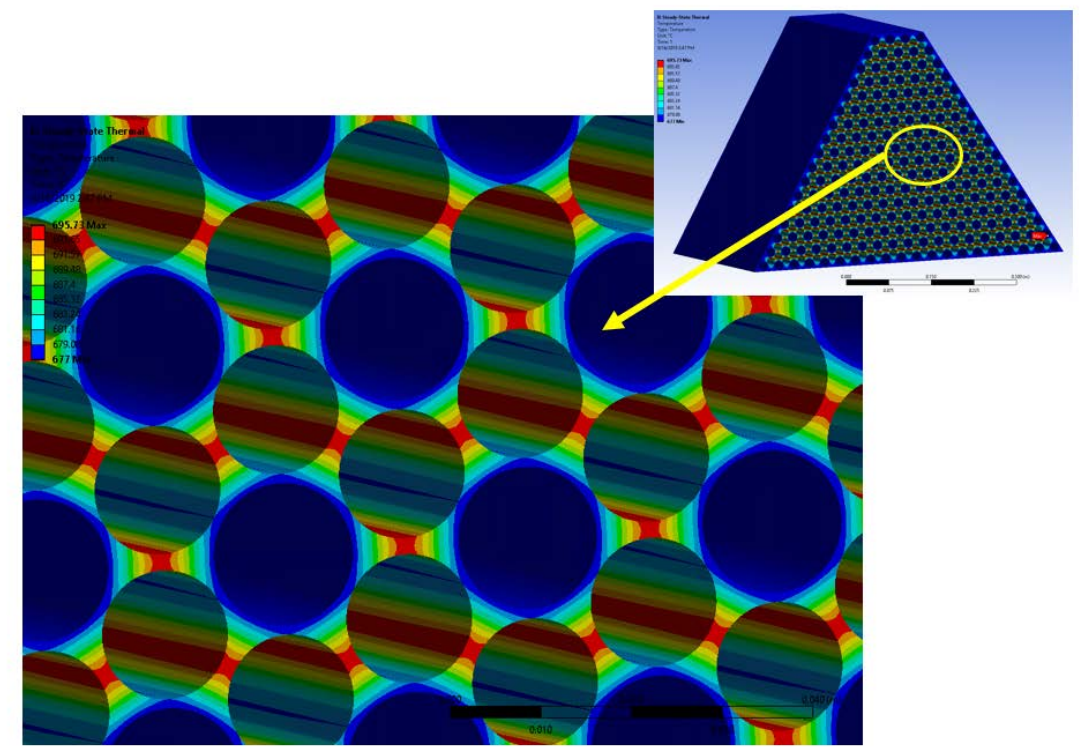

Figure 9.1 Temperature distribution at the mid-plane of the core block.

\subsection{Structural analysis}

Since the reactor studied is only a concept, design condition and service loads have not been specified, during the following discussion, only thermal stress, which is categorized as secondary stress is involved, while the primary stress, which is the results of pressure load, is assumed to be small due to the fact of the low reactor operating pressure. The following analysis of the FEA results will focus on how to apply design codes on the design and three aspects of FEA results were obtained and analyzed, they are

1. Stress intensity.

2. Equivalent stress based on Huddleston's theory.

3. Triaxiality factors (TF).

\subsubsection{Stress intensity}

Stress intensity is defined as the maximum of the differences between three principal stresses $\sigma_{1}, \sigma_{2}$, and $\sigma_{3}$, as shown in Equation (1) below:

$$
\text { stress intensity }=\max \left(\operatorname{abs}\left(\sigma_{1}-\sigma_{2}\right), a b s\left(\sigma_{2}-\sigma_{3}\right), a b s\left(\sigma_{1}-\sigma_{3}\right)\right)
$$

Figure 9.2 to Figure 9.4 shows the core block mid-plane stress intensity contour plots at start of the holding (1 second), 1000 hours, 10000 hours, 50000 hours and 100,000 hours. At start of the loading (1 second, Figure 9.2 (a)), the webs between fuel pin holes shows a stress intensity larger than 30MPa, the allowable stress values for SS316 at $700{ }^{\circ} \mathrm{C}$, this will not kill the design, as the thermal stress here is regarded as secondary stress, instead of primary stress. The contour plots also demonstrate that stress intensity reduce to less than 30MPa within 1000 hours (Figure 9.2 (b)), and by 10,000 hours, the stress intensities drop to less than 20MPa at most of regions, and after that, they continue to decrease, but at slower rates. Figure 9.4 
Initial study of notch sensitivity of Grade 91 using mechanisms motivated crystal plasticity finite element method

September 2019

(b), the average stress intensity transient plot, shows that most of the stress intensity drop occurs during the first 10,000 hours, after that it decreases slowly, from 50,000 hours to 100,000 hours, the average stress intensity changes less than $1 \mathrm{MPa}$.

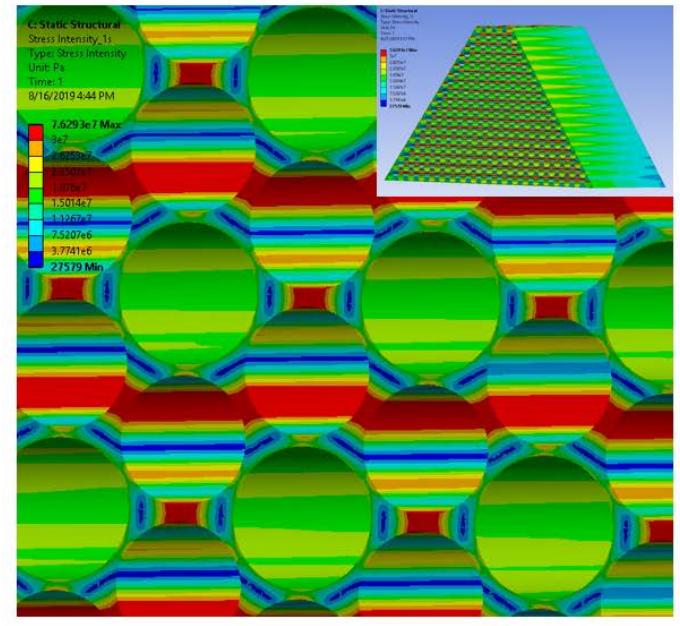

(a)

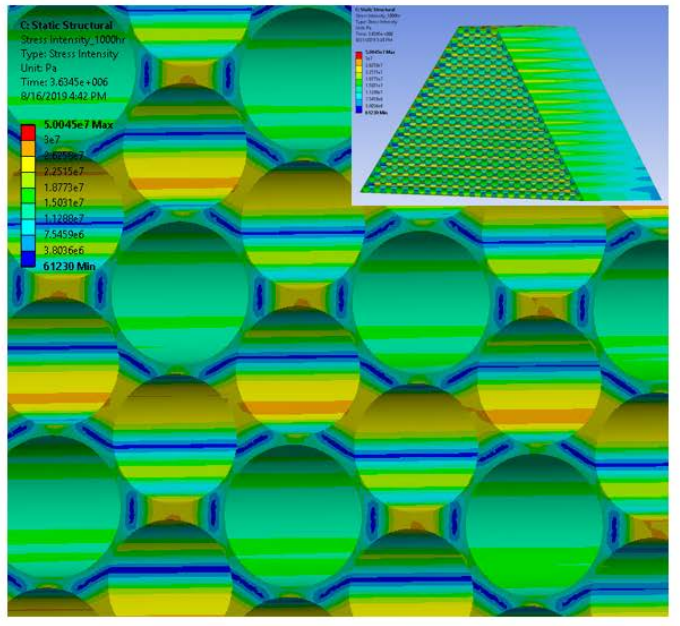

(b)

Figure 9.2 Stress intensity at (a) 1 second, and (b) 1,000 hours.

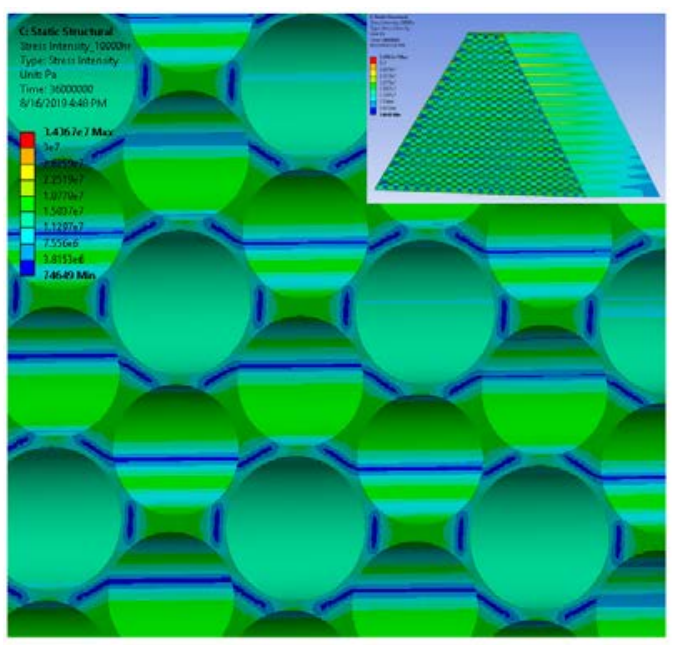

(a)

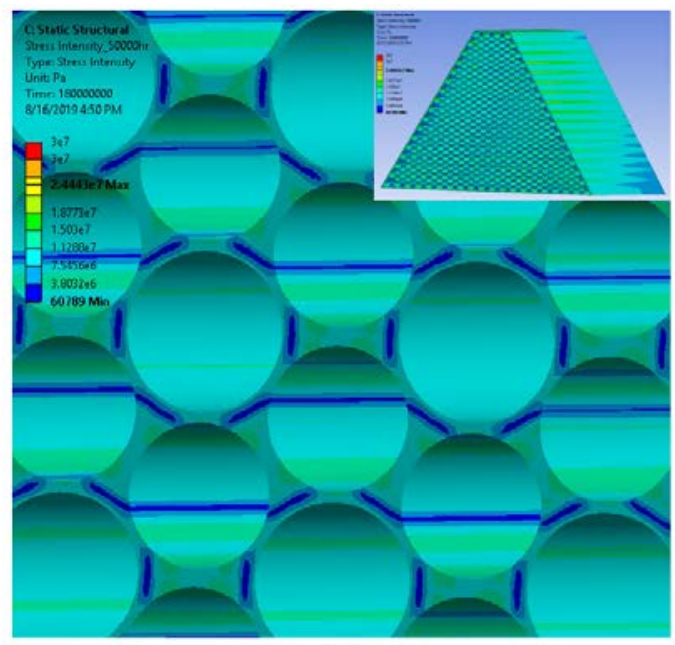

(b)

Figure 9.3 Stress intensity at (a) 10,000 hours, and (b) 50,000 hours. 
Initial study of notch sensitivity of Grade 91 using mechanisms motivated crystal plasticity finite element method

September 2019

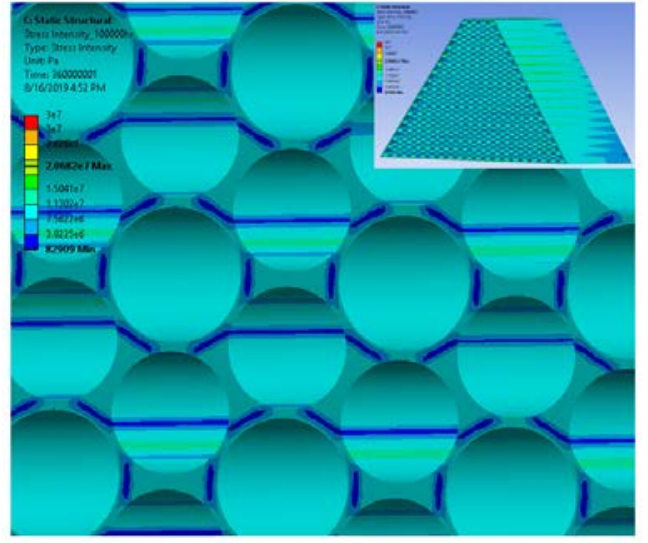

(a)

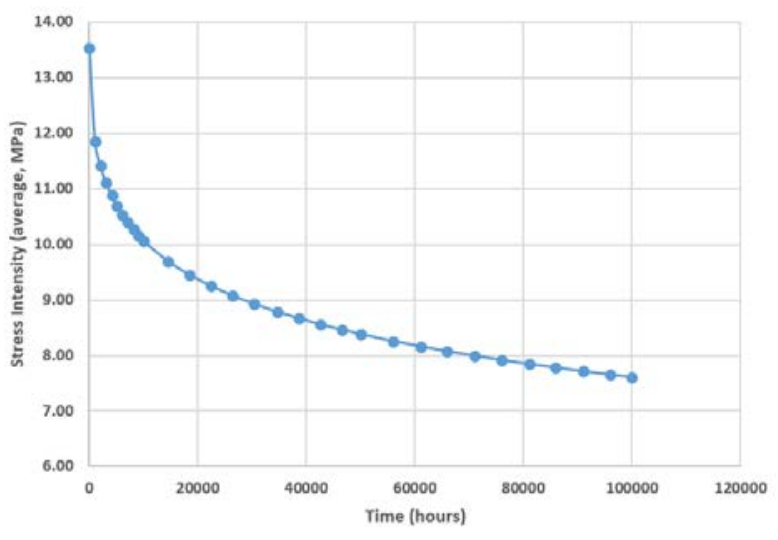

(b)

Figure 9.4 (a) Stress intensity at 50,000 hours, (b) average stress intensity transient.

The stress intensity here refers to stress intensity of thermal stress, and if the thermal load ramping rates are very low, the thermal stresses can also be regarded as secondary stress range $\mathrm{Q}_{\mathrm{R}}$, as the two stress extremes will be at the reactor starting low temperature soaking period and full load high temperature soaking period. The secondary stress range magnitude affects the core block structural integrity in a couple of ways ${ }^{[1]}$, (1) Whether the structure shakedown, (2) The maximum accumulated creep strain, (3) Cyclic load strain range and (4) Creep damage. As the reactor service loads and allowable deformation have not been specified, only item (1) and (4) are discussed in the following parts of the report.

Figure 9.2 (a) shows the thermal stress contour plot at the start of full load soaking, the plot shows the total stress magnitudes (max. 76MPa, after linearization they should decrease), considering possible residual stresses at starting temperature $677^{\circ} \mathrm{C}$ should be less than the yield strength $\left(\mathrm{S}_{\mathrm{y}}\right.$ appr. 100MPa at $677^{0} \mathrm{C}^{[1]}$ ), $\mathrm{QR}_{\mathrm{R} \text {,max }}$ is about $176 \mathrm{MPa}$. Figure 9.10 is from [1], parameters $\mathrm{X}$ and $\mathrm{Y}$ determines where the regimes the structure will operate, i.e., elastic, shakedown, alternating plastic or ratchetting. Based on the definite of $\mathrm{Y}$ value $\left(\mathrm{Y}=\mathrm{Q}_{\mathrm{R}} / \mathrm{S}_{\mathrm{y}}\right)$, according to [1], $\mathrm{Y}<2$, and as the primary load is small (low pressure), the core block will operate in either " $E$ " or " $\mathrm{S}_{2}$ " operating regime (elastic or shakedown regime) shown in Figure 9.10. 
Initial study of notch sensitivity of Grade 91 using mechanisms motivated crystal plasticity finite element method

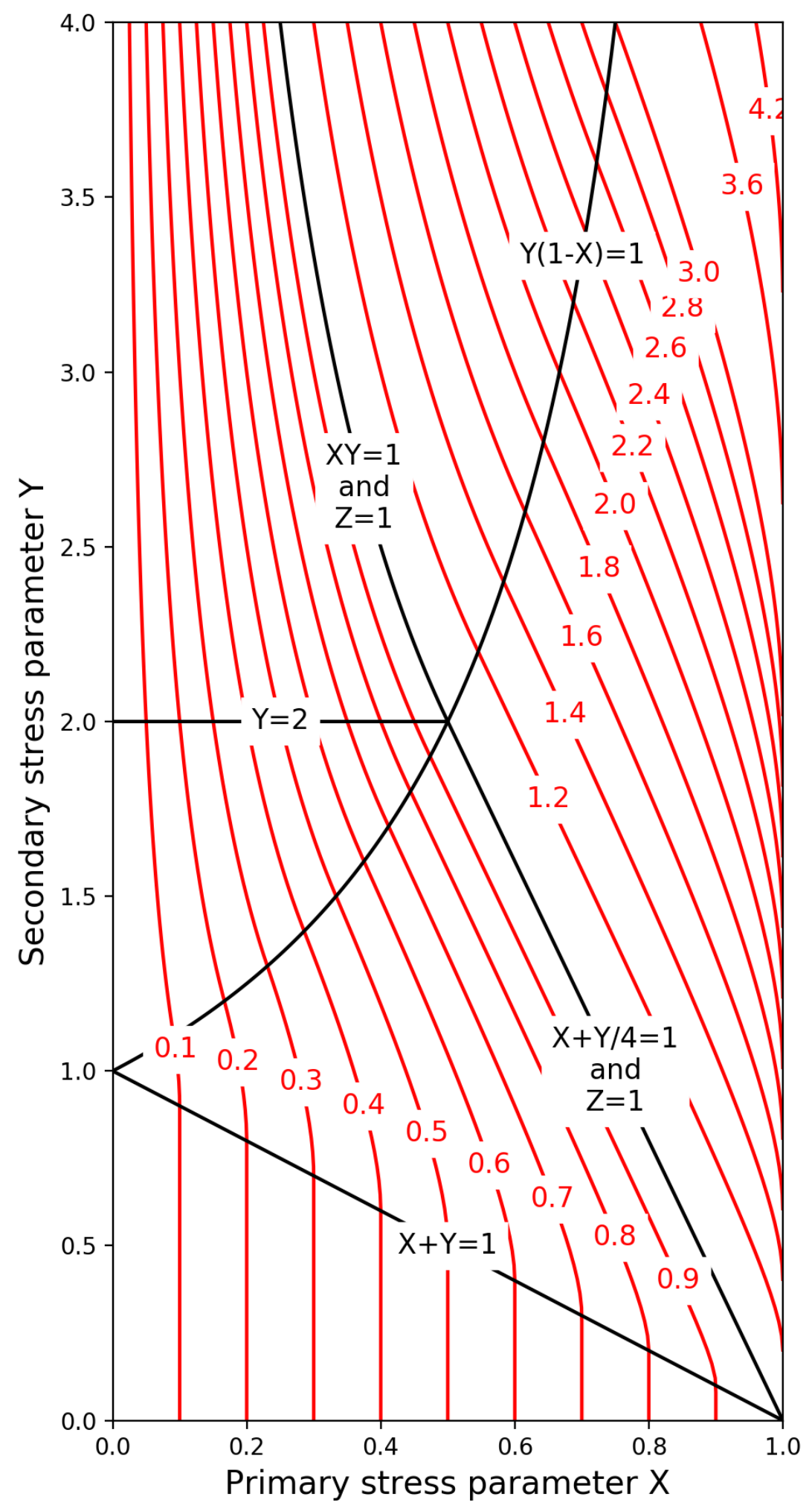

Figure 9.10 Effective Creep Stress Parameter Z ${ }^{[1]}$. 
Initial study of notch sensitivity of Grade 91 using mechanisms motivated crystal plasticity finite element method

September 2019

The other effects of $Q_{R}$ is on the creep damage, higher $Q_{R}$ leads to higher initial holding stress for either the cyclic load enhanced creep damage or un-disturbed stress relaxation creep damage ${ }^{[1]}$ when the creep damage is evaluated through time-fraction approach by elastic analysis.

\begin{tabular}{|c|c|c|c|}
\hline Time (hours) & $\begin{array}{c}\text { Max. Stress } \\
\text { Intensity (Mpa) }\end{array}$ & $\begin{array}{c}\text { Max. Intensity at } \\
\text { fuel pin-fuel pin } \\
\text { web (Mpa) }\end{array}$ & $\begin{array}{c}\text { Max. Stress Intensity } \\
\text { at fuel pin-heat pipe } \\
\text { web (Mpa) }\end{array}$ \\
\hline 0 & 76.3 & 40.5 & 28.9 \\
\hline 1000 & 50 & 27.2 & 23.6 \\
\hline 10,000 & 34.4 & 17.8 & 19.1 \\
\hline 50,000 & 24.4 & 12.9 & 14.9 \\
\hline 100,000 & 20.7 & 11.1 & 13.3 \\
\hline
\end{tabular}

Table 9.1 Stress intensity changes during 100,000 hour time period.

From the results shown in Figure 9.2 to Figure 9.4, the stress intensity keeps decreasing from 0 hours to 100,000 hours, and most of the stress intensity drop occurs within the first 1,000 hours, shown in Table 9.1. If elastic analysis approach ${ }^{[1]}$ were applied, the stress intensity results from 0 hour data frame would be utilized, even though stress relaxation is later involved, due to the fact that the stress relaxation would be based on uniaxial creep relaxation experiments and stress state effects would not be included and the creep damage estimated this way could be overly conservative. The next two sections will introduce stress state effects for creep damage calculation, one is for stress-based method (inelastic, Huddleston's method) and the other is for strain based method (ductility exhaustion), and applying them to future creep damage estimation for the core block could reduce the conservatism introduced by time fraction method.

\subsubsection{Equivalent stress based on Huddleston's theory}

The equivalent stress based on Huddleston's theory ${ }^{[3]}$ is defined as:

$$
\sigma_{e}=\bar{\sigma} \exp \left[C\left(\frac{J_{1}}{S_{S}}-1\right)\right]
$$

$$
\begin{aligned}
& \text { where } \\
& \begin{array}{l}
J_{1}=\sigma_{1}+\sigma_{2}+\sigma_{3} \\
S_{s}=\left[\sigma_{1}{ }^{2}+\sigma_{2}{ }^{2}+\sigma_{3}{ }^{2}\right]^{1 / 2} \\
\bar{\sigma}=\frac{1}{\sqrt{2}}\left[\begin{array}{l}
\left.\left(\sigma_{1}-\sigma_{2}\right)^{2}+\left(\sigma_{2}-\sigma_{3}\right)^{2}\right]^{1 / 2} \\
+\left(\sigma_{3}-\sigma_{1}\right)^{2}
\end{array}\right] \\
\text { and } \sigma_{i}(i=1,2 \text {, or 3) are the principal } \\
\text { stresses. The constant } C \text { is defined as } \\
\text { follows: } \\
\text { (a) For Types } 304 \text { and } 316 \text { stainless } \\
\text { steels, } C=0.24 . \\
\text { (b) For Alloy } 800 \mathrm{H}, C=0 . \\
\text { (c) For } 2 \frac{1}{4} \mathrm{Cr}-1 \mathrm{Mo} \text { and } 9 \mathrm{Cr}-1 \mathrm{Mo}-\mathrm{V} \text { : } \\
\text { (1) If } J_{1} / S_{s} \geq 1.0, C=0.16 ; \\
\text { (2) If } J_{1} / S_{s}<1.0, C=0 .
\end{array}
\end{aligned}
$$


Initial study of notch sensitivity of Grade 91 using mechanisms motivated crystal plasticity finite element method

September 2019

Figure 9.5 to Figure 9.7 shows the contour plots of the equivalent stresses calculated from FEA results based on Equation (2), they demonstrates that on the edges of the core block, there are some regions with high stresses, Table 9.2 shows the maximum equivalent stresses probed on the contour plots at the web locations, both the above mentioned figures and table show that the equivalent stresses decrease with time, and the total creep damage during 100,000 hour service life $<0.33$ (" 1 " means the material is damaged by creep). The creep rupture data of SS316 are from Table HBB-I_14.6B of [1], as the table only provide data till 300,000 hours with 27MPa at 700 ${ }^{0} \mathrm{C}$, no extrapolation was done for lower stress and higher rupture life, the creep damage values in Table 9.2 should be conservative.

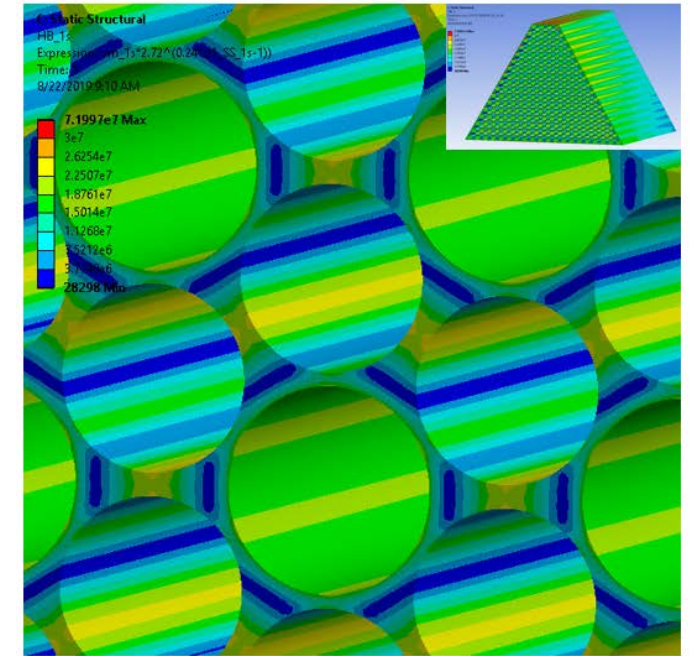

(a)

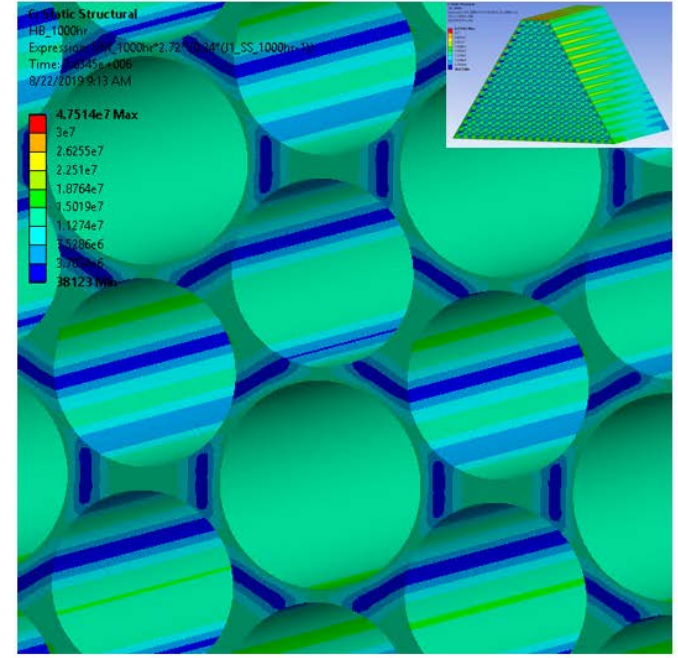

(b)

Figure 9.5 Equivalent stress at (a) 1 second, start of holding and (b) 1000 hours.

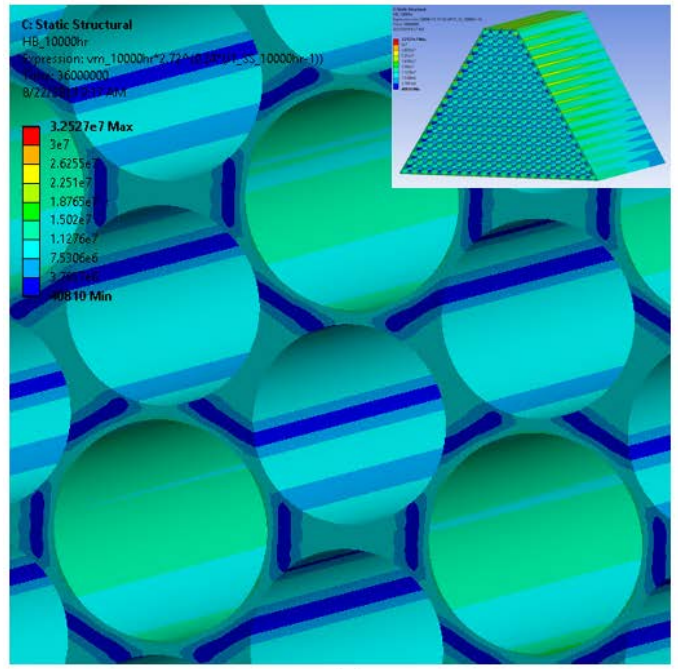

(a)

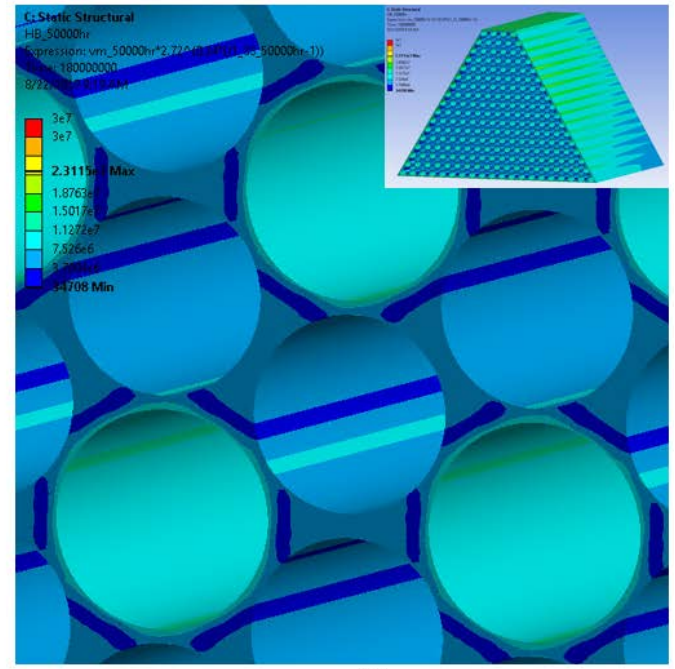

(b)

Figure 9.6 Equivalent stress at (a) 10,000 hours and (b) 50,000 hours. 
Initial study of notch sensitivity of Grade 91 using mechanisms motivated crystal plasticity finite element method

September 2019

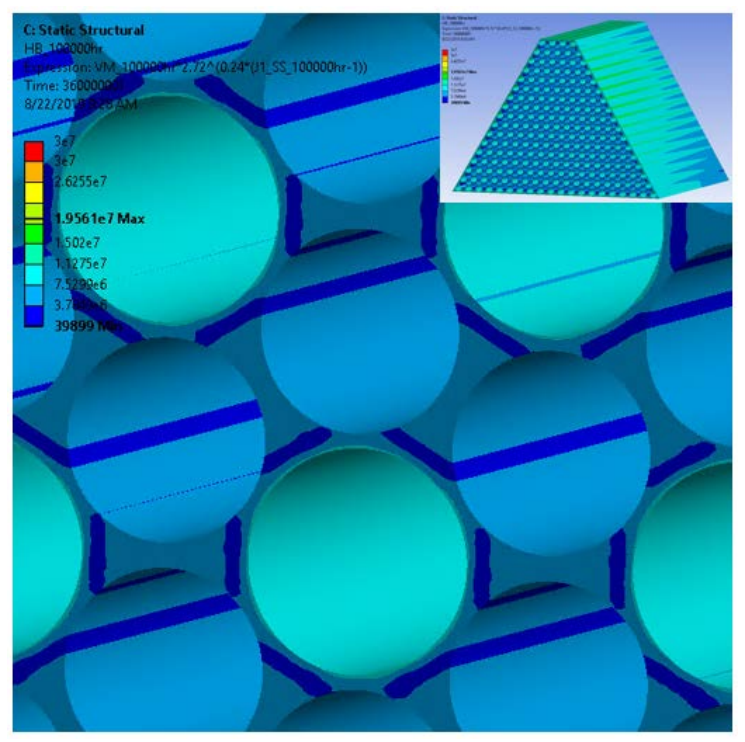

Figure 9.7 Equivalent stress at 100,000 hours.

\begin{tabular}{|c|c|c|c|c|c|}
\hline Time (hours) & $\begin{array}{c}\text { Equivalent stress } \\
\text { (webs, probed, Mpa) }\end{array}$ & $\mathbf{K}^{\prime}$ & $\begin{array}{c}\text { Stress for creep } \\
\text { damage calculation } \\
\text { (Mpa) }\end{array}$ & $\begin{array}{c}\text { Allowable creep } \\
\text { life (hours) }\end{array}$ & $\begin{array}{c}\text { Accumulated } \\
\text { creep damage }\end{array}$ \\
\hline 0 & 23.5 & 0.67 & 35.1 & 100,000 & 0.000 \\
\hline 1000 & 15 & 0.67 & 22.4 & 300,000 & 0.003 \\
\hline 10000 & 12 & 0.67 & 17.9 & 300,000 & 0.033 \\
\hline 50000 & 10 & 0.67 & 14.9 & 300,000 & 0.167 \\
\hline 100000 & 9 & 0.67 & 13.4 & 300,000 & 0.333 \\
\hline
\end{tabular}

Table 9.2 Maximum equivalent stresses and creep damage of the core block*.

*Based on the data from Table HBB0I-14.3A of [1], conservative estimation.

It is apparent that when the stress states were included, stress data in Table 9.2 are lower than those in Table 9.1 and the calculated creep damage is surely less than those stress-based time fraction method without stress state effects involved, so, application of Equation (2) will surely decrease the creep damage estimation conservatism introduced through elastic analysis.

One of the features of Equation (2) is that when $\mathrm{J}_{1} / \mathrm{S}_{\mathrm{s}}<1$, the exponential term in the equation will be less than 1, i.e., the Von Mise's stress, which is already lower than or equal to stress intensity, can be further reduced before being applied for creep damage calculations, that will surely decrease estimated creep damage and reduce the creep life assessment conservatism. Figure 9.8 shows an example, at $\mathrm{t}=1$ second, when the full thermal load is applied and before the creep relaxation starts. Stress intensity in the fuel pin -fuel pin webs is higher than 38MPa (shown in Figure 9.8 (a)), but the $J_{1} / S_{s}$ at those regions are less than 1, shown in blue color in Figure 9.8 (b), so the equivalent stress based on Equation (2) decreases to around 22MPa (shown in Figure 9.8 (c)), 
Initial study of notch sensitivity of Grade 91 using mechanisms motivated crystal plasticity finite element method

September 2019

surely the creep damage will be smaller and the estimated creep life will be larger. It would be expected that $\mathrm{J}_{1} / \mathrm{S}_{\mathrm{s}}$ could be regarded as one of the structure optimization parameters for future reactor design.

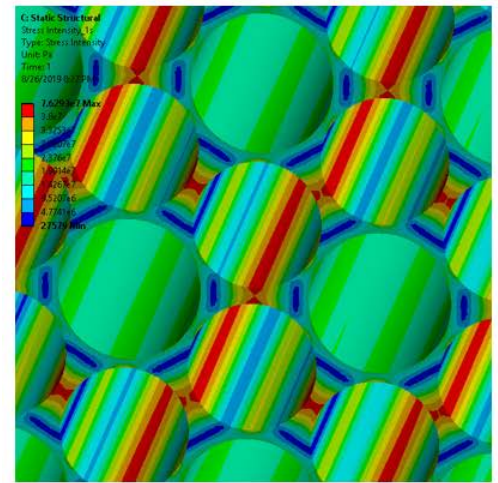

(a)

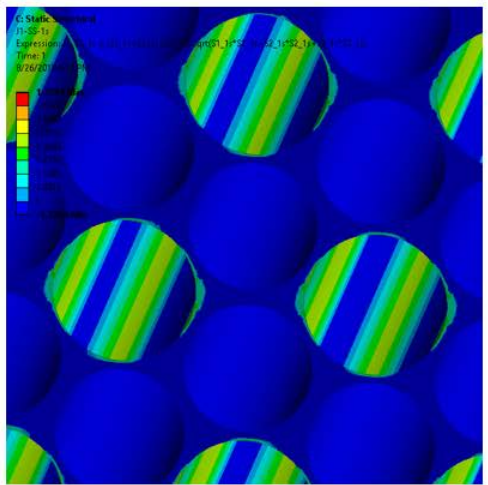

(b)

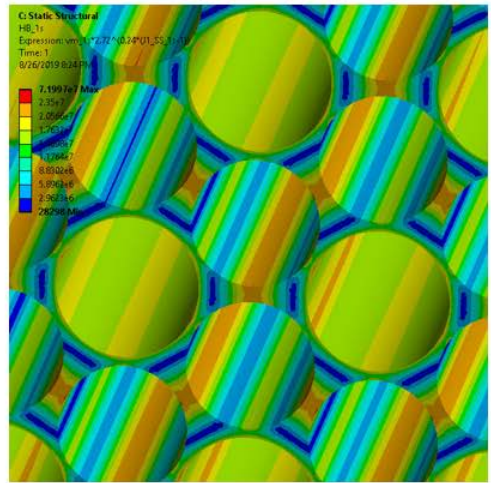

(c)

Figure 9.8 (a) stress intensity at $\mathrm{t}=1 \mathrm{~s}$, (b) $\mathrm{J}_{1} / \mathrm{S}_{\mathrm{s}}$ at $\mathrm{t}=1 \mathrm{~s}$ and (c) equivalent stress at $\mathrm{t}=1 \mathrm{~s}$.

\subsubsection{Triaxiality Factors}

The creep damage calculation mentioned so far is stress-based time-fraction approach based on Robinson's rule, in which the creep damage $\omega$ can be expressed as

$$
\omega=\int \frac{d t}{t_{r}(\sigma, T)}
$$

Where $t$ is time, $t_{r}$ is the time to rupture, a function of stress $\sigma$ and temperature $\mathrm{T}$.

An alternative way to calculate creep damage is strain-based ductility-exhaustion approach, in which the creep damage $\omega$ can be expressed as

$$
\omega=\int \frac{d \varepsilon_{c}}{\varepsilon_{f}^{*}}
$$

Where $\varepsilon_{\mathrm{c}}$ and $\varepsilon^{*} \mathrm{f}$ are uniaxial creep strain and multiaxial creep ductility, respectively.

Ductility exhaustion method for creep damage calculation has been adopted in EDF R5 code ${ }^{[4]}$, and there is evidence that it provides more accurate results than stress based time fraction $\operatorname{method}^{[5]}$. Triaxility factor (TF) is one of the factors determining creep fracture strain $\varepsilon_{\mathrm{f}}{ }_{\mathrm{f}}$ at different stress states. TF is defined as 


$$
T F=\frac{\left(\sigma_{1}+\sigma_{2}+\sigma_{3}\right)}{\sigma_{v m}}
$$

Where

$$
\begin{gathered}
\sigma_{v m}=\sqrt{\frac{\left(\sigma_{1}-\sigma_{2}\right)^{2}+\left(\sigma_{2}-\sigma_{3}\right)^{2}+\left(\sigma_{3}-\sigma_{1}\right)^{2}}{2}} \\
\sigma_{1}, \sigma_{2} \text { and } \sigma_{3} \text { are three principal stresses. }
\end{gathered}
$$

Multiaxial creep ductility models by Spindler ${ }^{[5]}$, Cocks and Ashby ${ }^{[6]}$, Manjoine ${ }^{[7]}$, Yatomi and Nikbin $^{[8]}$ and Manjoine ${ }^{[9]}$ all show that as long as the creep damage is through the mechanism of void nucleation, growth and connection, larger is the TF, lower is the $\varepsilon^{*} \mathrm{f}$; in other words, two samples made of the same material, the one with larger TF will show higher creep damage, even though the uniaxial creep fracture strains of the sample materials are the same. Till today, even though the modeling effects are for 3D TF situation, the experimental evidence supporting TF on creep life are all from experiments with 2D stress conditions, how the TF actually affects material creep life under true 3D stress condition and how to apply it to future ASME codes for reactor design is still under investigation (Mark Messner, 2019, personal communication).

Figure 9.7 to Figure 9.9 show the TF contour plots. Through the 100,000 hours service life, at the core block boundaries, there are high TF regions due to boundary effects. In the other regions, the TFs at the webs between fuel pin holes are less than 0, i.e., TF doesn't affect creep ductility and stress state's effect on creep damage can be neglected. Since those regions are under compression at high temperature and inelastic deformation occurs due to creep, when the reactor cools down, they will under tension and cracking could be an issue and under cyclic operation, i.e., the cracks may grow, and lead to component failure, the webs between fuel pin holes need more attention during manufacturing and NDE, to ensure no defects, i.e., inclusions, small cracks, in those regions. In the regions around heat pipe hole walls with $\mathrm{TF}>1$ (in orange color), they will see a reduction in creep ductility and creep life, compared with uniaxial testing samples. Figure 9.2 to Figure 9.4 shows the stress intensities in those regions are low, so, creep damage may not be an issue. 
Initial study of notch sensitivity of Grade 91 using mechanisms motivated crystal plasticity finite element method

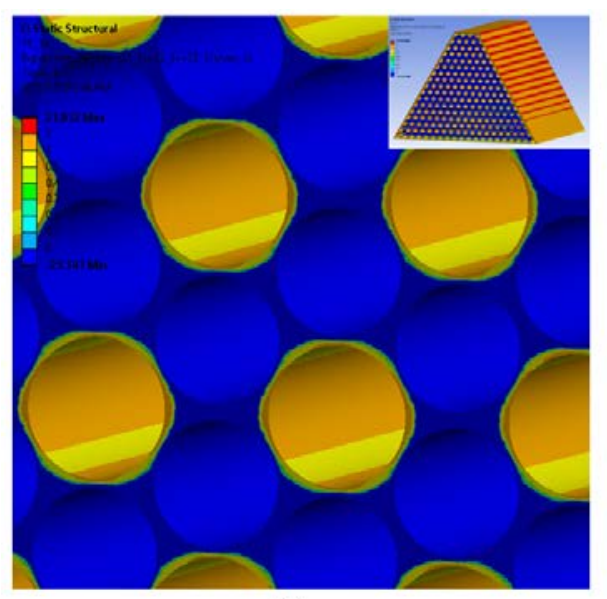

(a)

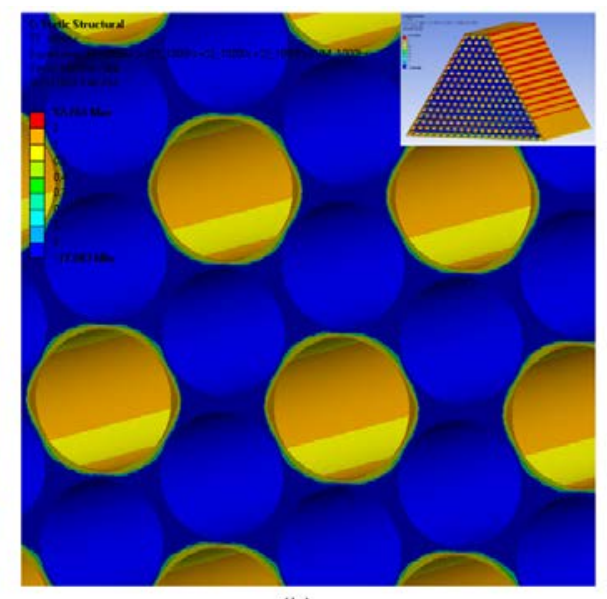

(b)

Figure 9.7 TF at (a) $1 \mathrm{~s}$, start of the holding and (b) 1,000 hours.

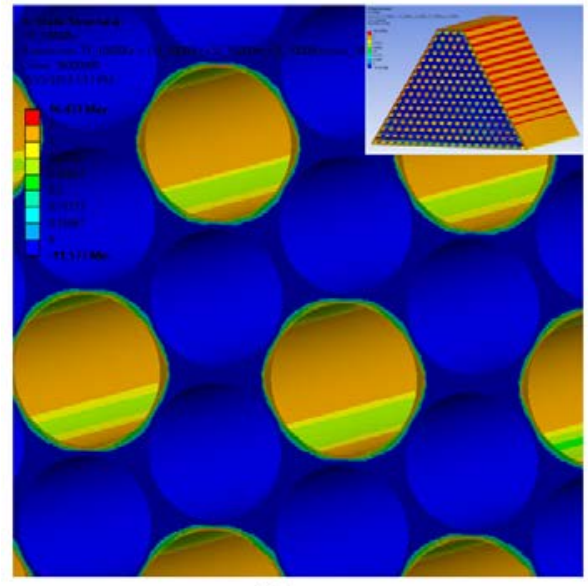

(a)

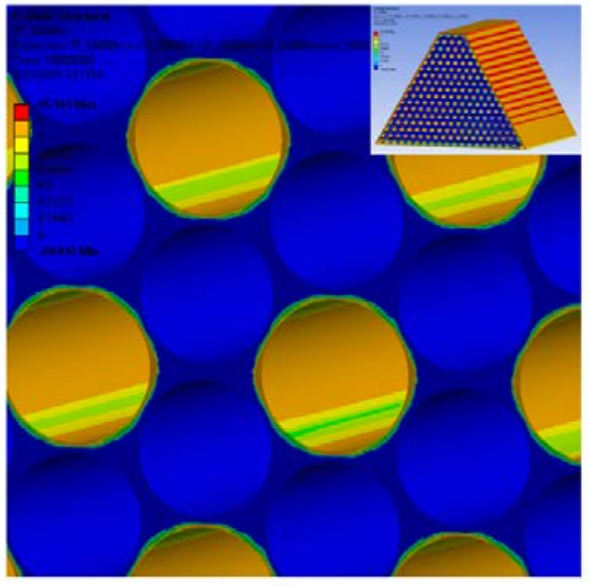

(b)

Figure 9.8 TF at (a) 10,000 hours and (b) 50,000 hours.

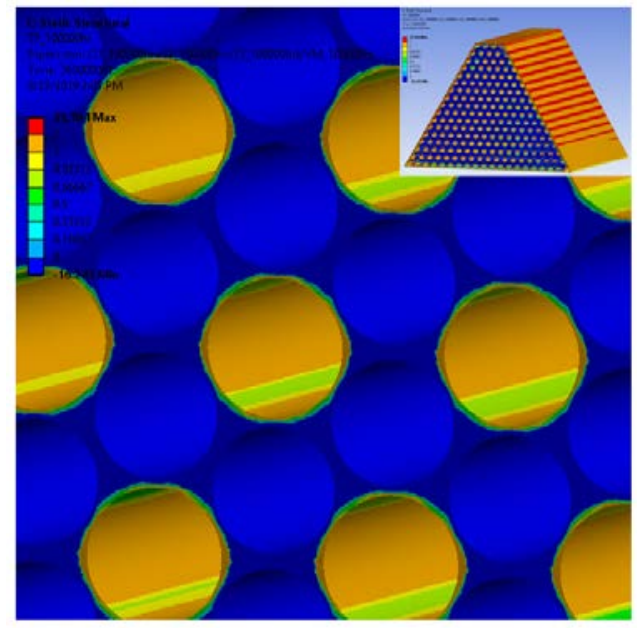

Figure 9.9 TF at 100,000 hours 
Initial study of notch sensitivity of Grade 91 using mechanisms motivated crystal plasticity finite element method

September 2019

\section{Conclusions}

Based on the results of this analysis, the following conclusions are drawn:

1. Secondary stress range (thermal stress + residual stress) is lower than twice the yield strength and the reactor will operation in the elastic regime or shakedown regime based on test B-1 of [1], and as the primary load (pressure load) is small, structural ratchetting is unlikely.

2. When stress state effects are included in equivalent stress calculation based on Huddleston's equation, the calculated creep damage will decrease and that reduces the conservatism introduced by other stress based time fraction approach.

3. Ductility exhaustion is another approach for creep damage calculation, triaxiality factor (TF) plays an important role on determining multiaxial creep ductility. The current reactor geometrical configuration leads to some regions around heat pipe hole walls with $\mathrm{TF}>1$, i.e., make those regions easier to be damaged by multiaxial creep.

4. The regions with $\mathrm{TF}<0$, i.e., those regions at the webs between fuel pin hole walls, are under compression at high temperature and creep deformation occurs, when the reactor cools down, those regions will be under tension and material cracking could be an issue. As service loads have not been specified, this issue will be left for future investigation.

\section{References}

1. ASME Boiler and Pressure Vessel Code 2017, Section III Div.5, American Society of Mechanical Engineering, Two Park Avenue, New York, NY 10016-5990

2. P.R. Mcclure, et.al. "Design of Megawatt Power Level Heat Pipe Reactors”, LANL report \# LA-UR-15-28840, Nov. 12, 2015.

3. R.L. Huddleston, "An Improved Multiaxial Creep-Rupture Strength Criterion”, Journal of Pressure Vessel Technology Nov.1985, 107(4):421-429

4. R. Hales, R.A. Ainsworth, “Multiaxial creep-fatigue rules”, Nuclear Engineering and Design 153 (1955) 257-264

5. M. W. Spindler, "An improved method for calculation of creep damage during creepfatigue cycling”, Materials Science and Technology, 2007, vol.23, 1461-1470.

6. A. F. Cocks, M.F. Ashby, “On Creep Fracture By Void Growth”, Progress in Material Science 1982, Vol.27. pp189 to 244

7. M.J. Manjoine, “Ductility Indices at Elevated Temperature”, Journal of Engineering Materials and Technology, 97(1975) 156-161.

8. M. Yatomi, K. M. Nikbin, "Numerical Prediction of Creep Crack Growth in Different Geometries Using Simplified Multiaxial Void Growth Model”, Materials at High Temperatures. 31(2014) 141-147

9. M. J. Manjoine, “Creep-Rupture Behavior of Weldments, Welding Journal, 61 (1982) $50-57$ 
Initial study of notch sensitivity of Grade 91 using mechanisms motivated crystal plasticity finite element method

September 2019

\section{Software}

- Ansys Mechanical, Version 19.2, Maintenance Release, UP20140420, Ansys Inc., Pittsburg, PA. 



\section{Argonne}

\section{Applied Materials Division}

Argonne National Laboratory

9700 South Cass Avenue, Bldg. 212

Argonne, IL 60439

www.anl.gov 